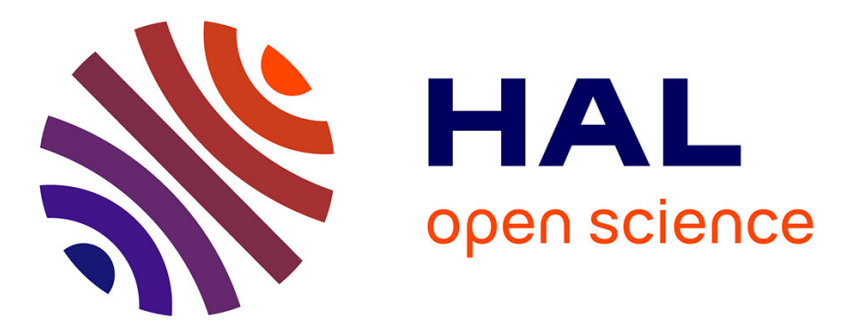

\title{
Organocatalyzed Sulfa-Michael Addition of Thiophenols on Trisubstituted $\alpha$-Fluoroacrylates, a Straightforward Access to Chiral Fluorinated Compounds
}

\author{
Xin Huang, Emilie David, Philippe Jubault, Tatiana Besset, Samuel \\ Couve-Bonnaire
}

\section{To cite this version:}

Xin Huang, Emilie David, Philippe Jubault, Tatiana Besset, Samuel Couve-Bonnaire. Organocatalyzed Sulfa-Michael Addition of Thiophenols on Trisubstituted $\alpha$-Fluoroacrylates, a Straightforward Access to Chiral Fluorinated Compounds. Journal of Organic Chemistry, 2020, 85 (21), pp.1405514067. 10.1021/acs.joc.0c02081 . hal-03004699

\section{HAL Id: hal-03004699 \\ https://hal.science/hal-03004699}

Submitted on 8 Dec 2020

HAL is a multi-disciplinary open access archive for the deposit and dissemination of scientific research documents, whether they are published or not. The documents may come from teaching and research institutions in France or abroad, or from public or private research centers.
L'archive ouverte pluridisciplinaire HAL, est destinée au dépôt et à la diffusion de documents scientifiques de niveau recherche, publiés ou non, émanant des établissements d'enseignement et de recherche français ou étrangers, des laboratoires publics ou privés. 


\title{
Organocatalyzed Sulfa-Michael Addition of Thiophenols on Trisubstituted $\alpha$ - Fluoroacrylates, a Straightforward Access to Chiral Fluorinated Compounds
}

\author{
Xin Huang, Emilie David, Philippe Jubault, Tatiana Besset and Samuel Couve-Bonnaire* \\ Normandie Université, COBRA, UMR 6014 et FR 3038, Université de Rouen, INSA Rouen, CNRS, \\ 1 rue Tesnière, 76821 Mont Saint-Aignan Cedex, France \\ E-mail: samuel.couve-bonnaire@insa-rouen.fr
}

\begin{abstract}
In this manuscript, a simple and efficient sulfa-Michael addition reaction of aryl thiols to trisubstituted $\alpha$-fluoro- $\alpha, \beta$-unsaturated esters both in racemic and, for the first time, in enantioselective version is reported. The commercially available dimer of cinchona derivatives $(\mathrm{DHQ})_{2} \mathrm{PYR}$ was used as catalyst. This strategy showed a great tolerance for various substrates and substituents, providing fair to excellent yields, moderate to excellent diastereoselectivities (2:1 to > $99: 1$ ) and low to good enantioselectivities (2 to $87 \%$ ). The reaction has been applied to the synthesis of fluorinated analogues of Diltiazem and Tiazesim, both therapeutic agents.
\end{abstract}

\section{Introduction}

Fluorine-containing compounds are widely used in various fields including polymers, pharmaceuticals and agrochemicals. ${ }^{1}$ Indeed, due to the highest electronegativity and the small size of the fluorine atom, the incorporation of one or several fluorine atoms into organic compounds often brings unique physical, chemical and biological properties. Considering the importance of chirality as well as the introduction of a fluorinated moiety into organic compounds, the quest for methods to create stereogenic fluorinated carbon centers with high enantioselectivity and catalytic efficiency is highly valuable. ${ }^{2}$ The asymmetric construction of stereogenic fluorinated carbon centers can be achieved by asymmetric direct fluorination or starting from already fluorinated molecules as prochiral $\mathrm{sp}^{2}$ substrates. In this context, organocatalysis emerged as a powerful tool in asymmetric synthesis ${ }^{3}$ and had a large impact on the development of asymmetric and catalytic conjugate additions of various nucleophiles to Michael acceptors for the construction of carbon-carbon and carbonheteroatom bonds. ${ }^{4}$ In the past few years, part of our research program was dedicated to easy and efficient accesses to 2-fluoroacrylates, ${ }^{5}$ which could serve as Michael acceptor. Surprisingly, whereas the Michael addition is a well-known strategy to obtain highlyfunctionalized products, only sporadic examples have been reported with fluoroalkene-type Michael acceptors, while the latter could be a suitable source of finefluorinated chemicals. For example, alkyl 2fluoroacrylates have been used as Michael acceptors since the sixties to the specific synthesis of relevant $\gamma$-fluoroglutamic acid. ${ }^{6}$ Other gem-fluoro-carbonyl $\left.\right|^{7}$ or -sulfonyl derivatives ${ }^{8}$ have also be submitted to Michael addition in the presence of various nucleophiles but, to our knowledge, no asymmetric reaction as well as no conjugate addition on trisubstituted fluoroalkenes substrates have been reported. Indeed, to the best of our knowledge, the asymmetric 1,4addition of nucleophiles with $\beta$-substituted- $\alpha$ fluoroacrylates has not been developed yet and only very few examples have been reported in racemic version to date. In an early report, Normant et al. studied the addition of lithium dimethylcuprate on $\alpha$-fluoro- $\alpha, \beta$-unsaturated ketones, aldehydes and esters. ${ }^{9}$ Both 1,2- and 1,4-adducts were formed and the ratios depend on the steric hindrance at the $\beta$-position of the substrate. Whereas the cuprates are wellknown to be 1,4 -regioselective,${ }^{10}$ the fluorine atom increased the electrophilicity of the geminal carbonyl moiety allowing the 1,2adduct formation in non-negligible amount and pointing out the unusual reactivity of fluorinated Michael acceptors. Even more surprisingly, in the case of $\beta$-substituted- $\alpha$-fluoroacrylates, no 1,4 -adduct was isolated because the enolate from 1,4-addition underwent a Claisen reaction 
to form a dimeric product with concomitant loss of fluorine atom (Scheme 1, Eq a). Later on, in their quest to fluorinated heterocycles, Schlosser et al. reported two examples of 1,4addition of thiophenols on the 2-fluoro-3methoxyacrylate, a specially designed Michael acceptor, using a catalytic amount of $t$-BuOK or one equivalent of $n$-BuLi as a base, affording the corresponding 1,4-adduct as a mixture of diastereoisomers in 3:1 to 3:2 ratios and in good yields (Scheme 1, Eq b). ${ }^{11}$ Considering the dearth of examples for the racemic and asymmetric conjugate addition of nucleophiles with fluoroacrylates, herein we report the SulfaMichael Addition $(S M A)^{4 c, 12}$ of thiophenol derivatives with a-fluoroacrylates both in racemic and enantioselective ways (Scheme 1, Eq. c).

Scheme 1. Conjugate addition on $\beta$ substituted- $\alpha$-fluoroacrylates: state of the art and this work

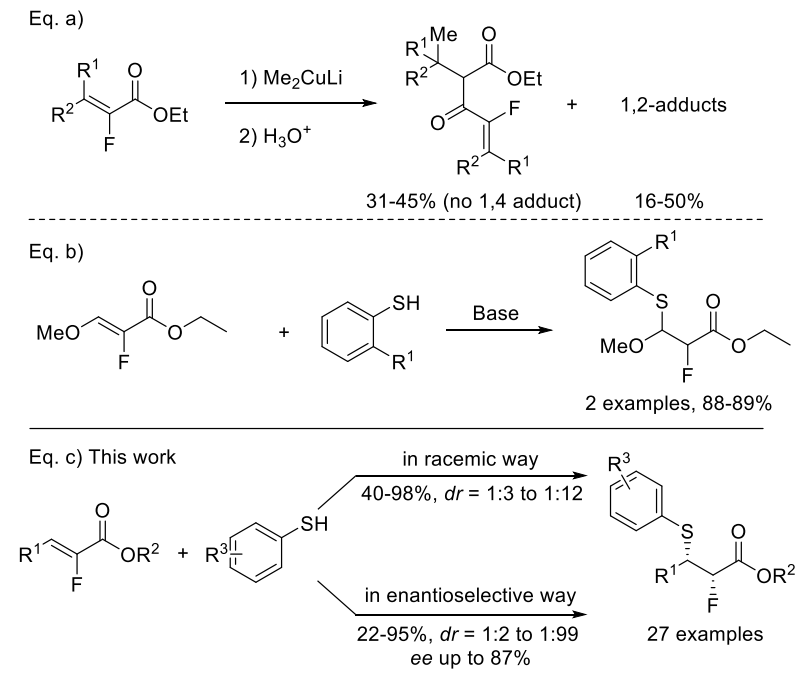

Two more general points highlighted the relevance of our study, i) sulfur containingcompounds $^{13}$ are very important as biochemical reagents as well as pharmaceutical agents and the one-pot formation of products bearing both sulfur and fluorine atoms could lead to interesting new structures towards biorelevant molecules; ii) whereas the asymmetric SMA reaction is known from a long time and has been studied with a large variety of Michael acceptor, ${ }^{4 c,}, 12 a$ only few organocatalytic studies have been reported with $\alpha, \beta$-unsaturated esters substituted in $\alpha^{-14}$ or in $\beta$-position, ${ }^{15}$ and even less with acrylates substituted in both $\alpha$ and $\beta$ positions, ${ }^{16}$ whereas in the latter two stereogenic centers could be concomitantly formed.

\section{Results and Discussion}

We chose the (Z)-ethyl 2-fluoro-5-phenylpent2-enoate (1a) as a model Michael-acceptor and the thiophenol as nucleophile to begin our study on SMA reaction. First investigations were carried out in a racemic version. SMA being usually efficiently carried out under basic conditions, ${ }^{4 c}$ we decided to consider the low pKa of thiophenol and use the weak base $\mathrm{NEt}_{3}$ as catalyst. Worthy of note whatever the precaution taken, during the SMA reactions, the oxidation of thiophenol occurred as a sidereaction making the isolation of 1,4-adducts difficult. To minimize the production of disulfide by-products, all the tests were performed in degassed solvents under argon. Traces of 1,4adducts were identified by ${ }^{19} \mathrm{~F}$ NMR (characteristic doublet of doublet corresponding to two diastereoisomers at $195.0 \mathrm{ppm}$ and $-197.0 \mathrm{ppm}$ ) when using triethylamine as a catalytic base in dichloromethane; the conversion was not improved when the reaction mixture was heated to reflux for a longer time (Table 1, entries 1-2). Among other solvents tested, acetonitrile appeared as the most suitable one, leading to $50 \%$ and $75 \%$ conversion at $22^{\circ} \mathrm{C}$ (room temperature) and $80^{\circ} \mathrm{C}$, respectively (Table 1, entries 3-4). 1,4-adduct 3 was isolated in moderate $37 \%$ yield due to difficulties to separate product $\mathbf{3}$ from remaining starting material and disulfide byproducts (Table 1, entry 4). The structure of 1,4-adduct was approved by ${ }^{1} \mathrm{H},{ }^{13} \mathrm{C},{ }^{19} \mathrm{~F}$ NMR experiments and also by mass spectrometry. Diastereoisomers were obtained in a $77 / 23$ ratio according to ${ }^{19} \mathrm{~F}$ NMR of the crude. The SMA reaction did not proceed in absence of triethylamine (Table 1, entry 5). Noteworthy that the use of sodium thiophenolate instead of the couple thiophenol/triethylamine was totally inefficient. ${ }^{17}$ Increasing the amount of thiophenol to 2 equivalents led to an improved $84 \%$ yield in 16 hours (Table 1, entry 6 ). With 2 equivalents of thiophenol, the amount of $\mathrm{NEt}_{3}$ could be reduced to 0.1 equivalent giving 
almost the same isolated yield and $d r$ (Table 1 , entry 7).

Table 1. Optimization of SMA reactions in racemic version

\begin{tabular}{|c|c|c|c|c|c|c|}
\hline entry & $\begin{array}{r}1 \mathrm{a} \\
\mathrm{X}\end{array}$ & $y$ & $\begin{array}{c}\text { 2A (x. Equiv.) } \\
\text { Solvent }\end{array}$ & $\mathrm{T}^{\circ} \mathrm{C}$ & $\begin{array}{l}\text { Conv. }(\%)^{a} \\
(\text { Yield }(\%))^{b}\end{array}$ & $D r^{c}$ \\
\hline 1 & 1.1 & 0.14 & $\mathrm{CH}_{2} \mathrm{Cl}_{2}$ & $22^{\circ} \mathrm{C}$ & traces & \\
\hline $2^{d}$ & 1.1 & 0.14 & $\mathrm{CH}_{2} \mathrm{Cl}_{2}$ & reflux & traces & \\
\hline 3 & 1.1 & 0.14 & $\mathrm{CH}_{3} \mathrm{CN}$ & $22^{\circ} \mathrm{C}$ & 50 & \\
\hline $4^{d}$ & 1.1 & 0.14 & $\mathrm{CH}_{3} \mathrm{CN}$ & $80^{\circ} \mathrm{C}$ & 75 (37) & $73: 27$ \\
\hline 5 & 1.1 & - & $\mathrm{CH}_{3} \mathrm{CN}$ & $80^{\circ} \mathrm{C}$ & 0 & \\
\hline 6 & 2 & 0.14 & $\mathrm{CH}_{3} \mathrm{CN}$ & $80^{\circ} \mathrm{C}$ & $98(84)$ & $77: 23$ \\
\hline 7 & 2 & 0.1 & $\mathrm{CH}_{3} \mathrm{CN}$ & $80^{\circ} \mathrm{C}$ & $94(81)$ & $76: 24$ \\
\hline 8 & 3 & 0.1 & - & $80^{\circ} \mathrm{C}$ & $91(77)$ & $74: 26$ \\
\hline 9 & 5 & 0.1 & - & $80^{\circ} \mathrm{C}$ & $100(89)$ & $76: 24$ \\
\hline 10 & 10 & 0.1 & - & $80^{\circ} \mathrm{C}$ & $100(91)$ & $77: 23$ \\
\hline
\end{tabular}

${ }^{a}$ Determined by ${ }^{19} \mathrm{~F}$ NMR. ${ }^{b}$ Isolated yield of mixture of diastereoisomers. ${ }^{C} D r$ determined by ${ }^{19} \mathrm{~F}$ NMR of the crude mixture. ${ }^{d} 24 \mathrm{~h}$ reaction.

We finally found this reaction could be performed in neat thiophenol (Table 1, entries 8-10). Comparing different equivalent of thiophenol, 5 equivalents of thiophenol gave full conversion and 1.4-adducts were isolated in $89 \%$ and $76: 24 d r$ (Table 1, entry 9). No real improvement was observed with 10 equivalents of thiophenol (Table 1, entry 10). The use of other tertiary amines such as $N, N$ diisopropylethylamine or 1,8-Diazabicyclo[5.4.0]undéc-7-ène led to lower isolated yields of reaction, while slightly improving the $d r{ }^{17}$ Having identified suitable conditions for racemic SMA reactions on a-fluoroacrylates, we rapidly turned our attention to the development of the corresponding asymmetric 1,4-additions screening chiral catalysts and temperatures (Table 2). All the reactions were performed in neat thiophenol according to the previous study. The use of natural cinchona alkaloids, ${ }^{18}$ as the cinchonine led to good conversion but no stereodifferenciation (Table 2 , entry 1). The use of quinine as catalyst allowed to obtain an enantioselectivity but with low $15 \%$ value. Enhancing the catalyst loading to $20 \mathrm{~mol} \%$ increased the conversion without positive influence on the enantioselectivity. Quinidine gave reverse enantioselectivity compared to quinine, albeit with lower conversion (Table 2, entry 4). The use of aryl ethers of bis-cinchona alkaloids ${ }^{19}(\mathrm{DHQ})_{2} \mathrm{PYR}$ gave full conversion, good diasteroisomeric ratio and allowed to significantly enhance the ee value to $66 \%$ (Table 2 , entry 5 ).

\section{Table 2. Optimization of asymmetric SMA reaction.}

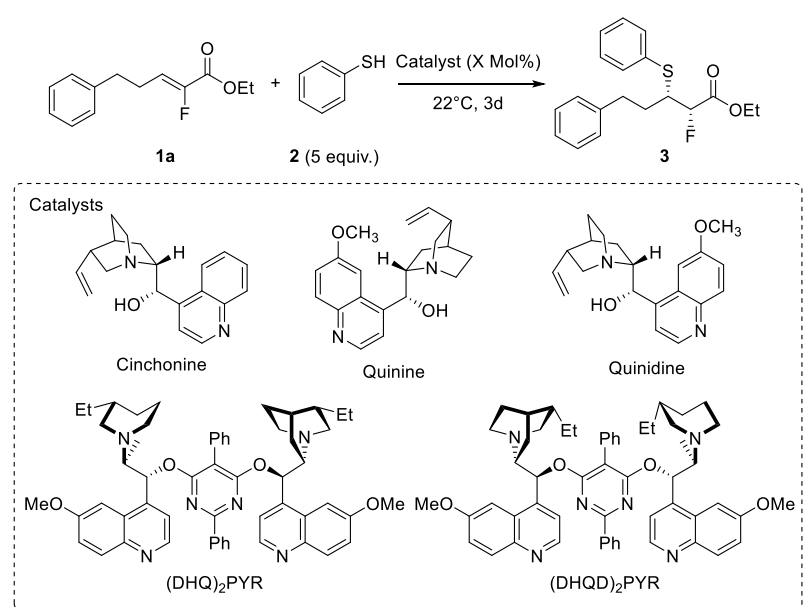

\begin{tabular}{|c|c|c|c|c|}
\hline Entry & Catalyst & $\mathrm{X}$ & $\begin{array}{l}\text { Conv. }(\%)^{a} \\
(\text { Yield }(\%))^{b}\end{array}$ & $D r c$ \\
\hline 1 & Cinchonine & 10 & 98 & $86: 14$ \\
\hline 2 & Quinine & 10 & 90 & $83: 17$ \\
\hline 3 & Quinine & 20 & 96 & $80: 20$ \\
\hline 4 & Quinidine & 10 & 85 & $85: 15$ \\
\hline 5 & $(\mathrm{DHQ})_{2} \mathrm{PYR}$ & 10 & 100 & $89: 11$ \\
\hline 6 & $(\mathrm{DHQD})_{2} \mathrm{PYR}$ & 10 & 63 & $88: 12$ \\
\hline 7 & $(\mathrm{DHQ})_{2} \mathrm{PYR}$ & 5 & 98 & $87: 13$ \\
\hline $8^{e}$ & $(\mathrm{DHQ})_{2} \mathrm{PYR}$ & 10 & 100 & $85: 15$ \\
\hline $9^{f}$ & $(\mathrm{DHQ})_{2} \mathrm{PYR}$ & 10 & $89(73)$ & $85: 15$ \\
\hline $10^{g}$ & $(\mathrm{DHQ})_{2} \mathrm{PYR}$ & 10 & 42 & $81: 19$ \\
\hline $11^{f, h}$ & $(\mathrm{DHQ})_{2} \mathrm{PYR}$ & 10 & $100(82)$ & $84: 16$ \\
\hline
\end{tabular}

${ }^{a}$ Determined by ${ }^{19} \mathrm{~F}$ NMR. ${ }^{b}$ Isolated yield of mixture of diastereoisomers. ${ }^{\circ} D r$ determined by ${ }^{19} \mathrm{~F}$ NMR of the crude mixture. ${ }^{d}$ Determined for major diastereoisomer by HPLC. ${ }^{e}$ Reaction carried out at $80^{\circ} \mathrm{C}, 16 \mathrm{~h}$ of reaction. ${ }^{f}$ Reaction carried out at $0{ }^{\circ} \mathrm{C}$. ${ }^{9}$ Reaction carried out at $22^{\circ} \mathrm{C}$. ${ }^{h} 5$ days of reaction.

The use of (DHQD) ${ }_{2} \mathrm{PYR}$ gave $72 \%$ ee but incomplete conversion (Table 2, entry 6). Decrease the catalyst loading to $5 \mathrm{~mol} \%$ decreased both the conversion and the enantiomeric excess (Table 2, entry 7 ). The reaction carried out at $80^{\circ} \mathrm{C}$ was complete after $16 \mathrm{~h}$ but gave a low $37 \%$ ee value (Table 2 , entry 8). Decrease the temperature allowed to increase the ee value to $70 \%$ and $73 \%$, at $0^{\circ} \mathrm{C}$ and $-20^{\circ} \mathrm{C}$, respectively. Nevertheless, the conversion was lower in these conditions (Table 2, entries 9-10). Finally, carrying out the reaction at $0^{\circ} \mathrm{C}$ for 5 days furnished full conversion with $71 \%$ of enantiomeric excess. 
Noteworthy that we tested more than fifteen catalysts, included other natural or modified cinchona alkaloids, chiral amines and also urea derivatives, ${ }^{20}$ including the Takemoto's urea ${ }^{20 a}$ which was already used successfully in SMA reaction, but the best results were obtained with $(\mathrm{DHQ})_{2} \mathrm{PYR}$ as a catalyst. ${ }^{17}$ Other dimers were also tested such as (DHQ) $)_{2} \mathrm{PHAL}$ and $(\mathrm{DHQ})_{2} \mathrm{AQN}$ but these catalysts gave lower ees. It is important to note that, in order to increase the enantiomeric excess, we studied the influence of about fifteen solvents on the reaction. None of them was more efficient that the reaction carried out in neat thiophenol. ${ }^{17}$ Finally, the use of bulkier ester than methyl or ethyl group such as iso-propyl or tert-butyl group led to lower yields and ees. ${ }^{17}$ $(D H Q)_{2} P Y R$, giving the best result in terms of conversion, diastereoisomeric and enantiomeric excesses, was selected to study the scope of the reaction with thiophenol acting both as reagent and solvent. With the optimized conditions in hand, the scope of SMA reaction of a variety of thiols on some $\alpha$ fluoroacrylates was investigated in both racemic and enantioselective ways (Scheme 2). We will first comment briefly about the racemic process before discussing more in details the asymmetric SMA reaction.

Mostly, the racemic way furnished good to excellent yield (61 to $98 \%$ yield) whatever the substituent contained in thiol or fluoroacrylate, except for compounds 17, 21 and 27, for which the yield is below $50 \%$ (40 to $46 \%)$. Diastereomeric ratios were obtained in a range from $71: 29$ to $92: 8$ with a major diastereoisomer in favor of the synconfiguration. This stereoselectivity was ascertained by X-ray analysis of cristals obtained from racemic product 13 (Figure 1, a). ${ }^{21 a}$

For asymmetric reaction, with the (Z)-ethyl 2fluoro-5-phenylpent-2-enoate $\mathbf{1 a}$, aryl thiols bearing electron-donating groups, such as methyl (in ortho, meta and para position), methoxy or amino, successfully furnished the desired products 5-9 in good to excellent yields $(74-95 \%)$ and good to very good diastereoselectivities $(81: 19$ to $95: 5)$. For unsubstituted 3 and ortho- or meta-substituted thioaryl 6-8, good ees were obtained (71 to $84 \%)$. However, thioaryl bearing a subtituent in para-position 4 ( $p-\mathrm{Br}), 5(p-\mathrm{OMe})$ and 9 ( $p-\mathrm{Me})$ were obtained in low to fair ee values: $23 \%$, $38 \%$ and $54 \%$, respectively. Unfortunately, 2thionaphtol, which proved to be the best arylthiol in the asymmetric 1,4-addition to cyclic enones, ${ }^{19 \mathrm{~b}}$ furnished the product $\mathbf{1 1}$ with only $29 \%$ of ee. The substrate scope could be extended to thiophene thiol to give 1,4-adduct 10 in good $73 \%$ yields, 88:12 dr ratio and $67 \%$ of ee. Additions of 4-bromothiophenol and naphthyl thiol to a fluorinated Michael acceptor bearing a nitro-aryl substituent occurred also in good yields and diastereoselectivities, but without enantioselectivities for products 12 and 13. Usually, diastereomeric ratios were better in enantioselective version of the reaction than in racemic one, except for compounds 4 and 11. Cyclohexyl a-fluoroacrylate 1c was also used in the SMA reaction, giving moderate to good yields (32 to $77 \%$ ), good to complete diastereoselectivities, from 69:31 for 15, bearing a bromine atom in para position, to $100: 0$ leading to the formation of a single diasteroisomer for the products 14 and 17, obtained from thiophenol and thiophene thiol, respectively. As for substrate 1a, substitution in para-position was detrimental for ee giving low value for products 15 and 16. Substitution in meta-position led also, in this case, to low $26 \%$ of ee. Product 18 obtained from 2aminothiophenol gave the best enantiomeric excess $87 \%$. The X-ray analysis of $\mathbf{1 8}$ crystals confirmed the syn configuration and revealed that the two stereogenic centers are both in $S$

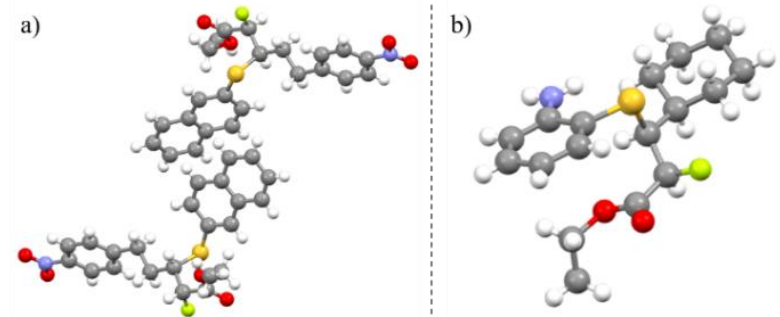
absolute configurations (Figure 1, b). ${ }^{21 b}$

Figure 1. a) X-ray of major diastereoisomers of 13 obtained in racemic way (mixture of syn enantiomers). b) X-ray of major enantiomer of 18 


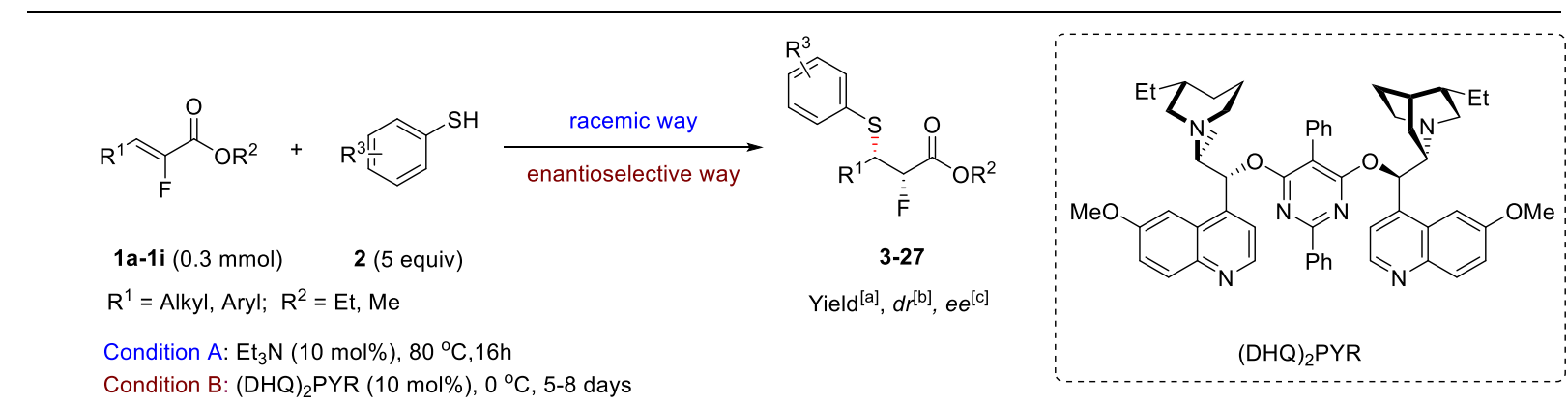

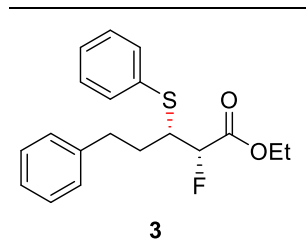

$91 \%, d r=76: 24$ $82 \%, d r=84: 16, e e=71 \%$ $73 \%, d r=87: 13, e e=72 \%$ [d]<smiles>CCOC(=O)C(F)[C@H](CCc1ccccc1)Sc1cccc(C)c1</smiles>

$84 \%, d r=72: 28$ $95 \%, d r=94: 6, e e=80 \%$<smiles>CCOC(=O)[C@H](F)[C@H](CCc1ccc([N+](=O)[O-])cc1)Sc1ccc2ccccc2c1</smiles>

$75 \%, d r=87: 13$ $55 \%, d r=84: 16, e e=8 \%$ [f]<smiles>CCOC(=O)[C@H](F)C(Sc1ccccc1N)C1CCCCC1</smiles>

$18^{[\mathrm{g}]}$

$75 \%, d r=80: 20$

$55 \%, d r=90: 10, e e=87 \%[\mathrm{e}]$ $51 \%, d r=91: 9, e e=85 \%{ }^{[\mathrm{e}][\mathrm{h}]}$<smiles>COC(=O)[C@H](F)[C@H](Sc1ccccc1)c1ccc(C)cc1</smiles>

23

$75 \%, d r=77: 23$ $36 \%, d r=88: 12, e e=69 \%$

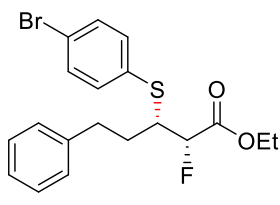

4

$98 \%, d r=73: 27$ $38 \%, d r=67: 33, e e=23 \%$<smiles>CCOC(=O)C(F)[C@H](CCc1ccccc1)Sc1ccc(C)cc1</smiles>

9

$67 \%, d r=74: 26$ $82 \%, d r=85: 15, e e=54 \%$

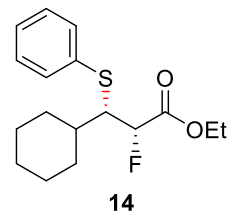

14

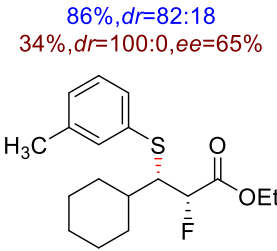

19

$67 \%, d r=80: 20$ $44 \%, d r=81: 19, e e=26 \%$<smiles>COC(=O)[C@H](F)[C@H](Sc1ccccc1)c1ccccc1C#N</smiles>

24

$62 \%, d r=89: 11^{[\mathrm{e}]}$ $78 \%, d r=87: 13, e e=52 \%[\mathrm{e}]$

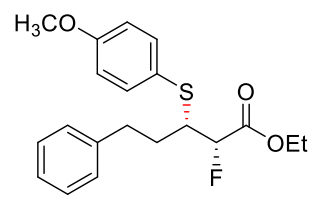

5

$87 \%, d r=84: 16$
$74 \%, d r=81: 19, e e=38 \%$

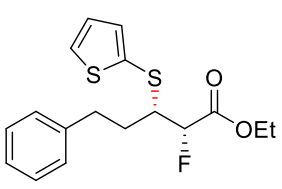

10

$83 \%, d r=83: 17$ $73 \%, d r=88: 12, e e=67 \%$

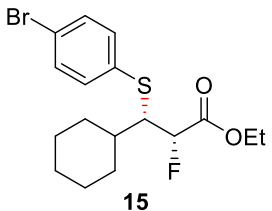

$76 \%, d r=71: 29$ $38 \%, d r=69: 31, e e=11 \%{ }^{[\mathrm{e}]}$

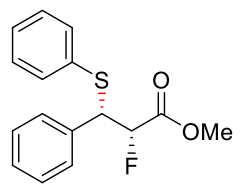

20

$61 \%, d r=83: 17$

$54 \%, d r=88: 12, e e=63 \%$<smiles>COC(=O)C(F)[C@H](Sc1ccccc1)c1cccc(C#N)c1</smiles>

25

$62 \%, d r=92: 8^{[\mathrm{e}]}$ $76 \%, d r=92: 8, e e=52 \%[$

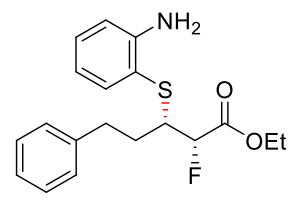

6

$91 \%, d r=80: 20^{[\mathrm{e}]}$ $95 \%, d r=90: 10, e e=84 \%$<smiles>CCOC(=O)[C@H](F)[C@H](CCc1ccccc1)Sc1ccc2ccccc2c1</smiles>

11

$84 \%, d r=80: 20$ $31 \%, d r=73: 27, e e=29 \%$<smiles>CCOC(=O)[C@H](F)[C@H](Sc1ccc(OC)cc1)C1CCCCC1</smiles>

16

$65 \%, d r=78: 22$

$77 \%, d r=80: 20, e e=32 \%$<smiles>Cc1ccccc1N</smiles><smiles>C=CCSC(c1ccccc1)C(F)C(=O)OC</smiles>

21

$46 \%, d r=87: 13^{[\mathrm{e}]}$ $22 \%, d r=91: 9, e e=51 \%{ }^{[\mathrm{e}]}$<smiles>COC(=O)[C@H](F)[C@H](Sc1ccccc1)c1ccc(C#N)cc1</smiles>

26

$64 \%, d r=81: 19^{[\mathrm{e}]}$ $70 \%, d r=100: 0, e e=68 \%[e]$

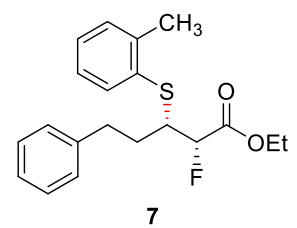

$91 \%, d r=73: 27$

$91 \%, d r=95: 5, e e=78 \%$<smiles>CCOC(=O)C(F)C(CCc1ccc([N+](=O)[O-])cc1)Sc1ccc(Br)cc1</smiles>

12

$73 \%, d r=82: 18$ $77 \%, d r=80: 20, e e=2 \%{ }^{[f]}$

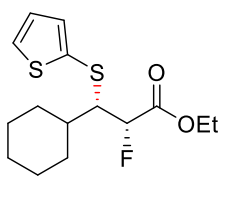

17

$43 \%, d r=82: 18$

$32 \%, d r=100: 0, e e=59 \%$ [e]

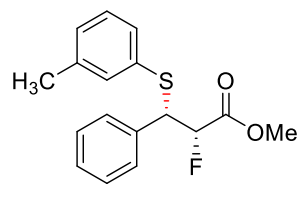

22

$89 \%, d r=85: 15$ $72 \%, d r=87: 13, e e=62 \%[\mathrm{e}]$<smiles>COC(=O)C(F)[C@H](Sc1ccccc1N)c1ccc(OC)cc1</smiles>

27

$40 \%, d r=87: 13$ $37 \%, d r=94: 6, e e=73 \%{ }^{[\mathrm{e}]}$

${ }^{a}$ Isolated yield of diastereoisomers. ${ }^{b}$ Determined by ${ }^{19} \mathrm{~F}$ NMR of crude mixture. ${ }^{c}$ Determined for major diastereoisomer by HPLC. ${ }^{d}$ Reaction performed on $1.5 \mathrm{mmol}$ scale of $\alpha$-fluoroacrylate 1a. ${ }^{e}$ Reaction carried out at $22^{\circ} \mathrm{C}$ (room temperature). 'Toluene as solvent. ${ }^{g}$ Absolute configuration of 18 was determined by single crystal X-Ray diffractometer; the two stereogenic centres are both in $S$ absolute configuration. ${ }^{h}$ Reaction performed on $1 \mathrm{mmol}$ scale of $\alpha$-fluoroacrylate $\mathbf{1 c}$. 
Then, we studied SMA addition on different aryl $\alpha$-fluoroacrylates (1d-i). Generally, with aryl $\alpha$ fluoroacrylates lower yields, compared to alkylfluoroacrylates 1a-c, were obtained in 1,4adducts $20-27$ (22 to $78 \%$ ), albeit with very good to excellent diastereoselectivities (87:13 to 100:0). Fair to good ee were obtained for these aryl substrates in a range from 51 to $73 \%$. a-Fluorocinnamates bearing a cyano group at the ortho-, meta-, and para-position of phenyl moiety gave the desired products 24-26 in similar results showing no significant substituent effects. Whereas the electronic feature of fluoroacrylates or aryl thiol does not seem to have an impact on the reaction, having a substituent in position para of the aryl thiol implied a lack of stereoselectivity meaning that the approach of the thiol is important in the enantioselective process. A kinetic study showed that the $d r$ and ee did not evolve from the beginning to the completion of the reaction. ${ }^{17}$ To get insight in the influence of the double bond geometry, we synthesized substrates 1a and 1d in $E$ configuration and submitted them to the SMA reaction. Interestingly, while similar yields and enantiomeric excesses for the major diastereoisomer were achieved, reversed diastereoisomer ratios were obtained starting with $(E)$-fluoroacrylates in place of its $(Z)$ congener, meaning that the face attack does not vary with the geometry of the double bond (Table 3). It has to be noted that for the reaction of $(E)-\mathbf{1 d}$, there was a little amount of $(Z)$ isomer in (E)-substrate (E/Z ratio: 90:10, (Z)-1d coming from the synthesis of starting material) explaining the lower $d r$ obtained (Table 3, entries 4 and 8). Ninomiya's research group reported the same kind of reversal $d r$ depending of double bond configuration with the SMA reaction between thiophenol and $\alpha, \beta$ trisubstituted acrylates in the presence of a catalytic amount of base and proposed a concerted process to explain their results. ${ }^{22}$ In our case, we probably have also a concerted mechanism with the thiol adding preferentially on the Si face of the $\beta$-position of the alkene, and the concomitant protonation on the opposite $R e$ face of the a-position. A substituent in para-position of the aryl-thiol should prevent the stereodifferenciation by steric hindrance. Two reactions on a larger scale were done with success starting from 1a (1.5 mmol) and 1c $(1 \mathrm{mmol})$, furnishing the desired products $\mathbf{3}$ and $\mathbf{1 8}$ in $73 \%$ of isolated yield, $87: 13$ of $d r, 72 \%$ of ee and $51 \%$ of isolated yield, $91: 9$ of $d r, 85 \%$ of ee, respectively.

\section{Table 3. influence of the double bond} geometry.

\begin{tabular}{|c|c|c|c|c|c|}
\hline $\begin{array}{l}\text { 1a: } R^{1}=P \\
\text { 1d: } R^{1}=\end{array}$ & $\begin{array}{l}\mathbf{2}( \\
\left.\mathrm{CH}_{2}\right)_{2}, \mathrm{R}^{2}=\mathrm{Et} \\
\mathrm{R}^{2}=\mathrm{Me}\end{array}$ & & & & \\
\hline Entry & Substrate & Cond. & $\begin{array}{l}\text { Yield } \\
(\%)^{[b]}\end{array}$ & $D r^{[c]}$ & $\begin{array}{c}E e^{[\mathrm{d}]} \\
(\%)\end{array}$ \\
\hline 1 & $(Z)-1 a$ & A & 91 & $76: 24$ & - \\
\hline 2 & (E) $-1 \mathbf{a}$ & A & 93 & $26: 74$ & - \\
\hline 3 & $(Z)-1 d$ & A & 61 & $87: 13$ & - \\
\hline 4 & $(E)-\mathbf{1} \mathbf{d}^{[\mathrm{e}]}$ & A & 74 & $33: 67$ & - \\
\hline 5 & $(Z)-1 \mathbf{a}$ & B & 82 & $84: 16$ & 71 \\
\hline 6 & $(E)-1 \mathbf{a}$ & B & 86 & $14: 86$ & 74 \\
\hline 7 & $(Z)-1 d$ & B & 54 & $88: 12$ & 63 \\
\hline 8 & $(E)-\mathbf{1} \mathbf{d}^{[\mathrm{e}]}$ & B & 56 & $24: 76$ & 51 \\
\hline
\end{tabular}

${ }^{a}$ Conditions $A=\mathrm{NEt}_{3}(10 \mathrm{~mol} \%), 80^{\circ} \mathrm{C}$, overnight: Conditions $\mathrm{B}=(\mathrm{DHQ})_{2} \mathrm{PYR}(10$ mol\%), $0^{\circ} \mathrm{C}, 8$ days. ${ }^{b}$ Isolated yield of mixture of diastereoisomers. ${ }^{\mathrm{c}} \mathrm{Dr}$ determined by ${ }^{19} \mathrm{~F}$ NMR of the crude mixture. ${ }^{d}$ Determined for major diastereoisomer by HPLC. ${ }^{e}$ Contaminated with $10 \%$ of $(Z)-\mathbf{1 d}$.

Finally, we applied our methodology to the synthesis of fluorinated analogues of biomolecules from both 21 and 27 compounds as starting materials. A two-step synthesis, ${ }^{23}$ a cyclisation under acid catalysis followed by an alkylation reaction, allowed to obtain, without variation of ees, fluoroanalogues of both Diltiazem, a calcium channel blocker, clinically used since 1974 as an antianginal and antihypertensive agent, and Tiazesim, an antidepressant agent (Scheme 3). ${ }^{24}$

\section{Conclusion}

In summary, we developed a simple and efficient sulfa-Michael addition reaction on $\beta$ subsituted- $\alpha$-fluoroacrylates in both racemic, and for the first time, in enantioselective version. This strategy shows great tolerance of various substrates and substituents, providing modest to excellent yields and moderate to good diastereoselectivities in racemic way (40$98 \%, d r=3: 1$ to $12: 1)$. 
Scheme 3. Synthesis of fluorinated analogues of biomolecules.

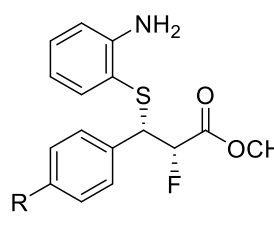

21: $\mathrm{R}=\mathrm{H}(e e=51 \%)$ $27: \mathrm{R}=$ OMe $(e e=73 \%)$

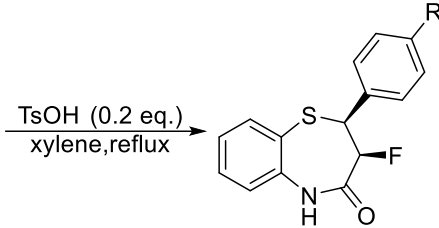

28: $R=H, 85 \%(e e=51 \%)$ 29: $R=$ OMe, $76 \%(e e=73 \%$

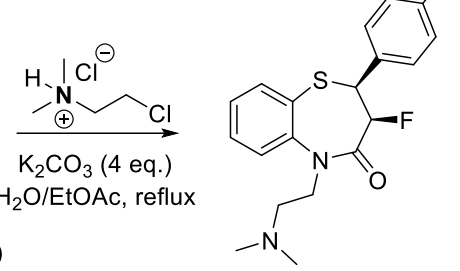

30: $\mathrm{R}=\mathrm{H}, 41 \%(e e=51 \%)$ 31: $\mathrm{R}=\mathrm{OMe}, 32 \%(e e=73 \%)$

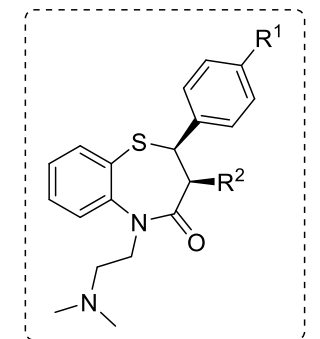

Diltiazem: $\mathrm{R}^{1}=\mathrm{OMe}, \mathrm{R}^{2}=\mathrm{OAC}$ Tiazesim : $\mathrm{R}^{1}=\mathrm{R}^{2}=\mathrm{H}$
In enantioselective way, good levels of reactivity, fair to excellent diastereoselectivities and moderate to good enantioselectivities were achieved by using commercially available $(\mathrm{DHQ})_{2} \mathrm{PYR}$ as catalyst $(22-95 \%, d r=2: 1$ to $>99: 1$, ee up to $87 \%$ ). Two fluorinated analogues of therapeutic agents were successfully synthesized using this SMA strategy. Other Michael addition with fluorinated Michael acceptors are presently under study in our laboratory.

\section{Experimental Section}

General Information: Unless otherwise mentioned, all the reagents were purchased from commercial source and used without further purification. All reactions were carried out in oven dried sealed tube under argon. Reaction temperatures were reported as the temperature of the bath surrounding the vessel. The dry solvents used were purified by distillation over the dry agents indicated in brackets and were transferred under argon: THF (Na, benzophenone), $\mathrm{CH}_{2} \mathrm{Cl}_{2}\left(\mathrm{CaH}_{2}\right)$, toluene ( $\mathrm{Na}$, benzophenone). Anhydrous DMF, $\mathrm{CH}_{3} \mathrm{CN}$, 1,4-dioxane, $\mathrm{Et}_{2} \mathrm{O}$ and $\mathrm{DME}$ were purchased from Acros Organics (Solvents Extra Dry over Molecular Sieve, Acroseal). Flash chromatography was carried out using Silicaflash P60 silica gel (40-60 mm); solvents used: $\mathrm{PE}=$ petroleum ether, $\mathrm{EA}=$ ethyl acetate. Melting points (Mp) were determined on a Fisher Scientific hot stage melting point apparatus and are uncorrected. ${ }^{1} \mathrm{H},{ }^{13} \mathrm{C}$ and ${ }^{19} \mathrm{~F}$ NMR spectra were recorded using a Bruker Avance-300 spectrometer operating at 300 $\mathrm{MHz}\left({ }^{1} \mathrm{H}\right), 75 \mathrm{MHz}\left({ }^{13} \mathrm{C}\right)$ and $282 \mathrm{MHz}\left({ }^{19} \mathrm{~F}\right)$, using $\mathrm{CDCl}_{3}$ as NMR solvent. The chemical shifts $(\delta)$ were calibrated on residual proton and carbon resonances of $\mathrm{CDCl}_{3}\left({ }^{1} \mathrm{H}, \delta=7.26\right.$ ppm and ${ }^{13} \mathrm{C}, \delta=77.2 \mathrm{ppm}$ ) or relative to external $\mathrm{CFCl}_{3}\left({ }^{19} \mathrm{~F}, \delta=0.0 \mathrm{ppm}\right)$. The multiplicity signals were indicated with the common abbreviations: s (singlet), d (doublet), $\mathrm{t}$ (triplet), $\mathrm{q}$ (quadruplet), $\mathrm{m}$ (multiplet) and br (broad) and the combinations thereof. IR spectra were recorded on Perkin Elmer Spectrum 100 FT IR spectrometer. High Resolution Mass Spectra (HRMS) were recorded on a JEOL AccuTof 4G spectrometer coupled to a GC HP Agilent 7890 . Enantiomeric excesses (ee) were determined by using a SpectraSYSTEM ${ }^{\text {TM }}$ HPLC equipped with a P1000XR pump, a UV1000 detector and a Rheodyne injector. See specific conditions detailed for each compound.

\section{General Procedure for Synthesis of Fluoroacrylates.}

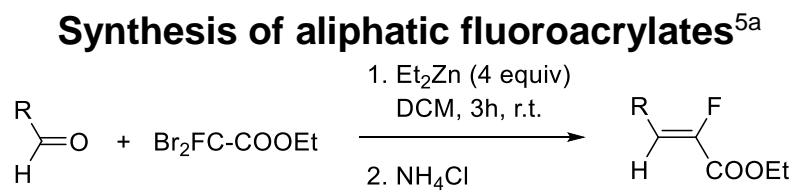

To an anhydrous $\mathrm{CH}_{2} \mathrm{Cl}_{2}$ solution $(10 \mathrm{~mL} / \mathrm{mmol}$ of aldehyde) of the appropriate aldehyde (1.0 equiv) and ethyl dibromofluoroacetate (2.0 equiv) was added diethylzinc ( $1 \mathrm{M}$ in hexane, 4.0 equiv) dropwise under argon. The reaction mixture was stirred during $3 \mathrm{~h}$ at $22^{\circ} \mathrm{C}$ (room temperature) (until anti alcohol was not detected by ${ }^{19} \mathrm{~F}$ NMR). The resulting solution was then poured into saturated $\mathrm{NH}_{4} \mathrm{Cl}, \mathrm{CH}_{2} \mathrm{Cl}_{2}$ was evaporated under vacuum, EtOAc was added, and the mixture was stirred for $15 \mathrm{~min}$. The remaining zinc salts were filtered off through a Büchner funnel. The heterogeneous 
resulting solution was extracted twice with $\mathrm{Et}_{2} \mathrm{O}$ $(2 \times 20 \mathrm{~mL} / \mathrm{mmol}$ of aldehyde), dried over $\mathrm{MgSO}_{4}$, and concentrated under reduced pressure and then purified by flash silica gel column chromatography.

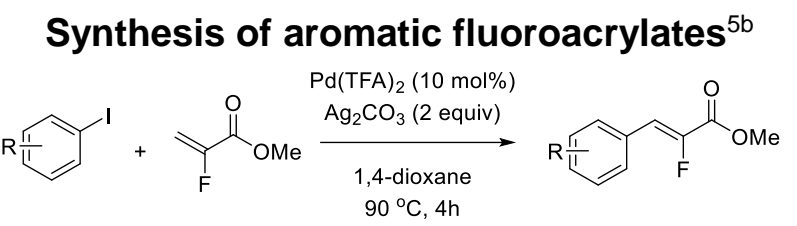

In a vial was added iodoarene (1.0 equiv), fluoroacrylate (1.5 equiv), $\mathrm{Ag}_{2} \mathrm{CO}_{3}$ (2.0 equiv) and $\operatorname{Pd}(\text { TFA })_{2}$ (10 mol\%). The vial was then filled with 1,4-dioxane $(5 \mathrm{~mL} / \mathrm{mmol}$ of iodoarene), then was heated to $90^{\circ} \mathrm{C}$ (oil bath) for $4 \mathrm{~h}$. The crude was filtrated over celite, washed with $\mathrm{EA}(3 \times 30 \mathrm{~mL} / \mathrm{mmol}$ of iodoarene), then solvent were evaporated. The crude was then purified by flash silica gel column chromatography.

\section{General Procedure for Sulfa-Michael Addition of Fluoroacrylates.}

\section{a) Racemic pathway:}

In an over-dried sealed tube, $\alpha$-fluoroacrylate 1 ( $0.3 \mathrm{mmol}$, 1equiv), thiol 2 ( $1.5 \mathrm{mmol}, 5$ equiv) followed by $\mathrm{NEt}_{3}(0.03 \mathrm{mmol}, 10 \mathrm{~mol} \%)$ were introduced, the tube was sealed and then freezed by liquid nitrogen, removed the air under high vacuum and introduced argon. The reaction mixture was stirred at $80^{\circ} \mathrm{C}$ (oil bath) for 16 hours and then was cooled to room temperature. The reaction mixture was purified by silica gel column chromatography (eluent: petroleum ether/EtOAc or petroleum ether $/ \mathrm{CH}_{2} \mathrm{Cl}_{2}$ ).

\section{b) Enantioselective pathway:}

In an over-dried sealed tube, a-fluoroacrylate ( $0.3 \mathrm{mmol}$, 1equiv), thiol (1.5 mmol, 5 equiv) followed by (DHQ) ${ }_{2}$ PYR $(0.03 \mathrm{mmol}, 10 \mathrm{~mol} \%)$ were introduced, the tube was sealed and then freezed by liquid nitrogen, removed the air under high vacuum and introduced argon. The reaction mixture was stirred at $0^{\circ} \mathrm{C}$ or at $22^{\circ} \mathrm{C}$ (room temperature) for five to eight days. Then the reaction mixture was purified by silica gel column chromatography (eluent: petroleum ether/EtOAc or petroleum ether $/ \mathrm{CH}_{2} \mathrm{Cl}_{2}$ ).

\section{Characterization Data for Products:}

(2S,3S)-ethyl

2-fluoro-5-phenyl-3-

(phenylthio)pentanoate (3). (Z)-ethyl 2-fluoro5 -phenylpent-2-enoate $(0.3 \mathrm{mmol}, 66.6 \mathrm{mg})$, with $(\mathrm{DHQ})_{2} \mathrm{PYR}(0.03 \mathrm{mmol}, 26.4 \mathrm{mg})$ were stirred in neat thiophenol $(1.5 \mathrm{mmol}, 165 \mathrm{mg})$ at $0{ }^{\circ} \mathrm{C}$ for 5 days according to the general procedure b. ${ }^{19} \mathrm{~F}$ NMR of crude product showed $d r=84: 16$. The crude was purified by silica gel column chromatography (petroleum ether/EtOAc, from 20/1 to 15/1, v/v) affording two diastereoisomers in $82 \%$ yield (major: 67 $\mathrm{mg}$ as a colorless oil; minor: $14 \mathrm{mg}$ as a colorless oil). $71 \%$ ee was obtained for the major diastereoisomer [determined by HPLC, IC column, Hept $/ i-\mathrm{PrOH}:$ 90/10, flow $=1$ $\mathrm{mL} / \mathrm{min}, \lambda=254 \mathrm{~nm}, \mathrm{t}$ (minor) $=6.810 \mathrm{~min}, \mathrm{t}$ (major) $=7.507 \mathrm{~min}$.]. The reaction performed on $1.5 \mathrm{mmol}$ of (Z)-ethyl 2-fluoro-5-phenylpent2-enoate led to $73 \%$ of isolated yield (major: $304 \mathrm{mg}$, major and minor mixture: $59 \mathrm{mg}$ ), $d r: 87: 13$ and ee: $72 \%$ (major diastereoisomer). The racemic product has been obtained following the procedure a (the same scale) in 91\% yield (major: $80 \mathrm{mg}$ as a colorless oil; minor: $11 \mathrm{mg}$ as a colorless oil) with $d r=76: 24$. $[\alpha]_{D}{ }^{20}=-18.0$ (c 1.23, $\left.\mathrm{CDCl}_{3}\right) ;{ }^{1} \mathrm{H}$ NMR $(300$ $\left.\mathrm{MHz}, \mathrm{CDCl}_{3}\right): \delta 7.37-7.34(\mathrm{~m}, 2 \mathrm{H}), 7.22-7.05$ $(\mathrm{m}, 8 \mathrm{H}), 4.97\left(\mathrm{dd}, 1 \mathrm{H},{ }^{2} J_{\mathrm{H}-\mathrm{F}}=48.0 \mathrm{~Hz}, J_{\mathrm{H}-\mathrm{H}}=\right.$ $3.0 \mathrm{~Hz}), 4.13-3.89(\mathrm{~m}, 2 \mathrm{H}), 3.49-3.33(\mathrm{~m}$, $1 \mathrm{H}), 2.92-2.68(\mathrm{~m}, 2 \mathrm{H}), 2.09-1.91(\mathrm{~m}, 2 \mathrm{H})$, $1.12\left(\mathrm{t}, 3 \mathrm{H}, J_{\mathrm{H}-\mathrm{H}}=9.0 \mathrm{~Hz}\right) .{ }^{19} \mathrm{~F}$ NMR $(282 \mathrm{MHz}$, $\left.\mathrm{CDCl}_{3}\right): \delta-197.0\left(\mathrm{dd},{ }^{2} J_{\mathrm{F}-\mathrm{H}}=48.0 \mathrm{~Hz},{ }^{3} \mathrm{~J}_{\mathrm{F}-\mathrm{H}}=\right.$ $28.2 \mathrm{~Hz}) .{ }^{13} \mathrm{C}\left\{{ }^{1} \mathrm{H}\right\} \mathrm{NMR}\left(75 \mathrm{MHz}, \mathrm{CDCl}_{3}\right): \delta$ $167.9\left(\mathrm{~d},{ }^{2} \mathrm{~J}_{\mathrm{C}-\mathrm{F}}=22.5 \mathrm{~Hz}\right), 140.7,133.8,132.9$, 129.0, 128.5, 128.5, 127.6, 126.2, 90.5 (d, ${ }^{1} J_{C}-$ $\mathrm{F}=195.0 \mathrm{~Hz}), 61.7,50.5\left(\mathrm{~d},{ }^{2} \mathrm{~J}_{\mathrm{C}-\mathrm{F}}=22.5 \mathrm{~Hz}\right)$, 33.4, 33.2, 14.0. IR: 2980, 1761, 1737, 1475, 1266, 1212, 1103, 1024, 745, 692, 557, 491 $\mathrm{cm}^{-1}$. HRMS $\left(\mathrm{API}^{+}\right) \mathrm{m} / \mathrm{z}:[\mathrm{M}+\mathrm{H}]^{+}$calcd for $\mathrm{C}_{19} \mathrm{H}_{22} \mathrm{FO}_{2} \mathrm{~S}: 333.1325$, found: 333.1319.

\section{(2S,3R)-ethyl}

2-fluoro-5-phenyl-3 (phenylthio)pentanoate (anti-3). (E)-ethyl 2fluoro-5-phenylpent-2-enoate $(0.3 \mathrm{mmol}, 66.6$ $\mathrm{mg})$, with $(\mathrm{DHQ})_{2} \mathrm{PYR}(0.03 \mathrm{mmol}, 26.4 \mathrm{mg})$ were stirred in neat thiophenol $(1.5 \mathrm{mmol}, 165$ $\mathrm{mg}$ ) at $0{ }^{\circ} \mathrm{C}$ for 5 days according to the general procedure $\mathbf{b} .{ }^{19} \mathrm{~F}$ NMR of crude product showed $d r=14: 86$. The crude was purified by silica gel 
column chromatography (petroleum ether/EtOAc, from $20 / 1$ to $15 / 1$, v/v) affording two diastereoisomers in $86 \%$ yield (major: 39 mg as a colorless oil; major and minor mixture: $47 \mathrm{mg}$ ). $74 \%$ ee was obtained for the major diastereoisomer [determined by HPLC, IC column, Hept $/ i-\mathrm{PrOH}: 90 / 10$, flow $=1 \mathrm{~mL} / \mathrm{min}$, $\lambda=230 \mathrm{~nm}$, $\mathrm{t}$ (minor) $=5.340 \mathrm{~min}, \mathrm{t}$ (major) $=$ $5.803 \mathrm{~min}$.]. The racemic product has been obtained following the procedure a (the same scale) in $93 \%$ yield (major: $72 \mathrm{mg}$ as a colorless oil; minor: $21 \mathrm{mg}$ as a colorless oil) with $d r=$ 26:74.

${ }^{1} \mathrm{H}$ NMR $\left(300 \mathrm{MHz}, \mathrm{CDCl}_{3}\right): \delta 7.40-7.37(\mathrm{~m}$, $2 \mathrm{H}), 7.25-7.09(\mathrm{~m}, 8 \mathrm{H}), 4.80\left(\mathrm{dd}, 1 \mathrm{H},{ }^{2} J_{\mathrm{H}-\mathrm{F}}=\right.$ $\left.48.0 \mathrm{~Hz}, J_{\mathrm{H}-\mathrm{H}}=6.0 \mathrm{~Hz}\right), 4.07\left(\mathrm{q}, 2 \mathrm{H}, J_{\mathrm{H}-\mathrm{H}}=6.0\right.$ $\mathrm{Hz}), 3.42-3.31(\mathrm{~m}, 1 \mathrm{H}), 2.94-2.70(\mathrm{~m}, 2 \mathrm{H})$, $1.94-1.87(\mathrm{~m}, 2 \mathrm{H}), 1.13\left(\mathrm{t}, 3 \mathrm{H}, J_{\mathrm{H}-\mathrm{H}}=9.0 \mathrm{~Hz}\right)$. ${ }^{19} \mathrm{~F}$ NMR (282 MHz, CDCl 3 ): $\delta-195.7$ (dd, ${ }^{2} J_{F-H}$ $\left.=48.0 \mathrm{~Hz},{ }^{3} \mathrm{~J}_{\mathrm{F}-\mathrm{H}}=22.6 \mathrm{~Hz}\right) \cdot{ }^{13} \mathrm{C}\left\{{ }^{1} \mathrm{H}\right\}$ NMR $(75$ $\left.\mathrm{MHz}, \mathrm{CDCl}_{3}\right): \delta 168.1\left(\mathrm{~d},{ }^{2} \mathrm{~J}_{\mathrm{C}-\mathrm{F}}=22.5 \mathrm{~Hz}\right.$ ), $140.8,133.2,132.9,129.2,128.5,127.9$, 126.2, $90.3\left(\mathrm{~d},{ }^{1} \mathrm{~J}_{\mathrm{C}-\mathrm{F}}=195.0 \mathrm{~Hz}\right), 61.8,49.5(\mathrm{~d}$, ${ }^{2} J_{C-F}=15.0 \mathrm{~Hz}$ ), 32.8, 30.6, 14.1 .

(2S,3S)-ethyl 3-((4-bromophenyl)thio)-2-fluoro5-phenylpentanoate (4). (Z)-ethyl 2-fluoro-5phenylpent-2-enoate $(0.3 \mathrm{mmol}, 66.6 \mathrm{mg})$, with (DHQ) ${ }_{2}$ PYR $(0.03 \mathrm{mmol}, 26.4 \mathrm{mg})$ were stirred in neat 4-bromobenzenethiol $(1.5 \mathrm{mmol}, 281$ $\mathrm{mg}$ ) at $0{ }^{\circ} \mathrm{C}$ for 5 days according to the general procedure b. ${ }^{19} \mathrm{~F}$ NMR of crude product showed $d r=67: 33$. The crude was purified by silica gel column chromatography (petroleum ether/EtOAc, from $15 / 1$ to $10 / 1, v / v$ ) affording two diastereoisomers in $38 \%$ yield (major: 38 mg as a white solid; minor: $9 \mathrm{mg}$ as a white solid). $23 \%$ ee was obtained for the major diastereoisomer [determined by HPLC, IC column, Hept/i-PrOH: 98/2, flow $=1 \mathrm{~mL} / \mathrm{min}, \lambda$ $=254 \mathrm{~nm}, \mathrm{t}($ minor $)=9.810 \mathrm{~min}, \mathrm{t}$ (major) $=$ $11.183 \mathrm{~min}$.$] . The racemic product has been$ obtained following the procedure a (the same scale) in $98 \%$ yield (major: $107 \mathrm{mg}$ as a white solid; minor: $13 \mathrm{mg}$ as a white solid) with $d r=$ 73:27.

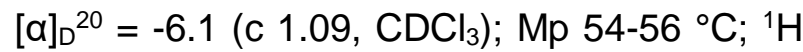
$\operatorname{NMR}\left(300 \mathrm{MHz}, \mathrm{CDCl}_{3}\right)$ : $\delta 7.34-7.31(\mathrm{~m}, 2 \mathrm{H})$, $7.22-7.06(\mathrm{~m}, 7 \mathrm{H}), 4.97\left(\mathrm{dd}, 1 \mathrm{H},{ }^{2} \mathrm{~J}_{\mathrm{H}-\mathrm{F}}=51.0\right.$ $\left.\mathrm{Hz}, J_{\mathrm{H}-\mathrm{H}}=3.0 \mathrm{~Hz}\right), 4.13-4.02(\mathrm{~m}, 2 \mathrm{H}), 3.41-$ $3.29(\mathrm{~m}, 1 \mathrm{H}), 2.85-2.73(\mathrm{~m}, 2 \mathrm{H}), 2.07-1.93$ $(\mathrm{m}, 2 \mathrm{H}), 1.17\left(\mathrm{t}, 3 \mathrm{H}, J_{\mathrm{H}-\mathrm{H}}=6.0 \mathrm{~Hz}\right) .{ }^{19} \mathrm{~F} \mathrm{NMR}$ $\left(282 \mathrm{MHz}, \mathrm{CDCl}_{3}\right): \delta-197.2\left(\mathrm{dd},{ }^{2} \mathrm{~J}_{\mathrm{F}-\mathrm{H}}=51.0\right.$ $\left.\mathrm{Hz},{ }^{3} \mathrm{~J}_{\mathrm{F}-\mathrm{H}}=31.0 \mathrm{~Hz}\right) \cdot{ }^{13} \mathrm{C}\left\{{ }^{1} \mathrm{H}\right\}$ NMR $(75 \mathrm{MHz}$, $\left.\mathrm{CDCl}_{3}\right): \delta 167.8\left(\mathrm{~d},{ }^{2} J_{\mathrm{C}-\mathrm{F}}=22.5 \mathrm{~Hz}\right), 140.4$, $134.5,132.8,132.0,128.6,128.5,126.3$, $122.0,90.6\left(\mathrm{~d},{ }^{1} \mathrm{~J}_{\mathrm{C}-\mathrm{F}}=187.5 \mathrm{~Hz}\right), 61.8,50.4(\mathrm{~d}$, $\left.{ }^{2} J_{\mathrm{C}-\mathrm{F}}=15.0 \mathrm{~Hz}\right), 33.3,33.1,14.1$. IR: 2920 , 1757, 1474, 1209, 1091, 815, 695, 585, 478 $\mathrm{cm}^{-1}$. HRMS $\left(\mathrm{API}^{+}\right) \mathrm{m} / \mathrm{z}:[\mathrm{M}+\mathrm{H}]^{+}$calcd for $\mathrm{C}_{19} \mathrm{H}_{21} \mathrm{BrFO}_{2} \mathrm{~S}: 411.0430$, found: 411.0429 .

(2S,3S)-ethyl 2-fluoro-3-((4methoxyphenyl)thio)-5-phenylpentanoate (5). (Z)-ethyl 2-fluoro-5-phenylpent-2-enoate $(0.3$ $\mathrm{mmol}, 66.6 \mathrm{mg})$, with $(\mathrm{DHQ})_{2} \mathrm{PYR}(0.03 \mathrm{mmol}$, $26.4 \mathrm{mg})$ were stirred in neat 4methoxybenzenethiol $(1.5 \mathrm{mmol}, 210 \mathrm{mg})$ at 0 ${ }^{\circ} \mathrm{C}$ for 5 days according to the general procedure b. ${ }^{19} \mathrm{~F}$ NMR of crude product showed $d r=81: 19$. The crude was purified by silica gel column chromatography (petroleum ether/EtOAc, from 49/1 to $19 / 1, \mathrm{v} / \mathrm{v}$ ) affording two diastereoisomers in $74 \%$ yield (major: 71 $\mathrm{mg}$ as a colorless oil; minor: $9 \mathrm{mg}$ as a colorless oil; major and minor mixture: $12 \mathrm{mg}$ ). $38 \%$ ee was obtained for the major diastereoisomer [determined by HPLC, IC column, Hept/i-PrOH: $90 / 10$, flow $=1 \mathrm{~mL} / \mathrm{min}, \lambda=254 \mathrm{~nm}, \mathrm{t}$ (minor) $=$ $8.783 \mathrm{~min}, \mathrm{t}$ (major) $=9.857 \mathrm{~min}$.]. The racemic product has been obtained following the procedure a (the same scale) in $87 \%$ yield (major: $78 \mathrm{mg}$ as a colorless oil; minor: $19 \mathrm{mg}$ as a colorless oil) with $d r=84: 16$.

$[\alpha]_{D}{ }^{20}=-10.4$ (c $\left.0.57, \mathrm{CDCl}_{3}\right) ;{ }^{1} \mathrm{H}$ NMR $(300$ $\left.\mathrm{MHz}, \mathrm{CDCl}_{3}\right): \delta 7.36-7.33(\mathrm{~m}, 2 \mathrm{H}), 7.19-7.06$ $(\mathrm{m}, 5 \mathrm{H}), 6.75-6.73(\mathrm{~m}, 2 \mathrm{H}), 4.92\left(\mathrm{~d}, 1 \mathrm{H},{ }^{2} \mathrm{~J}_{\mathrm{H}-\mathrm{F}}\right.$ $=48.0 \mathrm{~Hz}), 4.16-4.01(\mathrm{~m}, 2 \mathrm{H}), 3.71(\mathrm{~S}, 3 \mathrm{H})$, $3.27-3.17(\mathrm{~m}, 1 \mathrm{H}), 2.89-2.70(\mathrm{~m}, 2 \mathrm{H}), 1.99$ $-1.88(\mathrm{~m}, 2 \mathrm{H}), 1.17\left(\mathrm{t}, 3 \mathrm{H}, \mathrm{J}_{\mathrm{H}-\mathrm{H}}=6.0 \mathrm{~Hz}\right) .{ }^{19} \mathrm{~F}$ NMR (282 MHz, $\left.\mathrm{CDCl}_{3}\right): \delta-197.1$ (dd, ${ }^{2} J_{\mathrm{F}-\mathrm{H}}=$ $\left.48.0 \mathrm{~Hz},{ }^{3} \mathrm{~J}_{\mathrm{F}-\mathrm{H}}=28.2 \mathrm{~Hz}\right) \cdot{ }^{13} \mathrm{C}\left\{{ }^{1} \mathrm{H}\right\}$ NMR $(75$ $\left.\mathrm{MHz}, \mathrm{CDCl}_{3}\right): \delta 168.1\left(\mathrm{~d},{ }^{2} \mathrm{~J}_{\mathrm{C}-\mathrm{F}}=30.0 \mathrm{~Hz}\right)$, $159.9,140.9,136.2,128.6,128.5,126.2$, 123.4, 114.5, $90.8\left(\mathrm{~d},{ }^{1} \mathrm{~J}_{\mathrm{C}-\mathrm{F}}=187.5 \mathrm{~Hz}\right), 61.7$, $55.4,51.1\left(\mathrm{~d},{ }^{1} \mathrm{~J}_{\mathrm{C}-\mathrm{F}}=22.5 \mathrm{~Hz}\right), 33.2,33.0,14.1$. IR: 2940, 1760, 1455, 1494, 1245, 1026, 829, 699, 525, $493 \mathrm{~cm}^{-1}$. HRMS (API $\left.{ }^{+}\right) \mathrm{m} / \mathrm{z}:[\mathrm{M}+\mathrm{H}]^{+}$ calcd for $\mathrm{C}_{20} \mathrm{H}_{24} \mathrm{FO}_{3} \mathrm{~S}$ : 363.1430, found: 363.1426. 
(2S,3S)-ethyl 3-((2-aminophenyl)thio)-2-fluoro5-phenylpentanoate (6). (Z)-ethyl 2-fluoro-5phenylpent-2-enoate $(0.3 \mathrm{mmol}, 66.6 \mathrm{mg})$, with (DHQ) ${ }_{2}$ PYR (0.03 mmol, $\left.26.4 \mathrm{mg}\right)$ were stirred in neat 2-aminobenzenethiol $(1.5 \mathrm{mmol}, 188$ $\mathrm{mg}$ ) at $0^{\circ} \mathrm{C}$ for 5 days according to the general procedure $\mathbf{b} .{ }^{19} \mathrm{~F}$ NMR of crude product showed $d r=90: 10$. The crude was purified by silica gel column chromatography (petroleum ether/EtOAc, from 10/1 to 8/1, v/v) affording two diastereoisomers in 95\% yield (major: 89 mg as a yellow oil; major and minor mixture: 11 $\mathrm{mg}$. $84 \%$ ee was obtained for the major diastereoisomer [determined by HPLC, IC column, Hept $/ i-\mathrm{PrOH}$ : 90/10, flow $=1 \mathrm{~mL} / \mathrm{min}$, $\lambda=230 \mathrm{~nm}, \mathrm{t}$ (minor) $=11.380 \mathrm{~min}, \mathrm{t}$ (major) $=$ $15.850 \mathrm{~min}$.$] . The racemic product has been$ obtained following the procedure a the same scale) in $91 \%$ yield (major: $61 \mathrm{mg}$ as a yellow oil; major and minor mixture: $34 \mathrm{mg}$ ) with $d r=$ $80: 20$.

$[\alpha]_{D}{ }^{20}=+13.4\left(\mathrm{c} 1.08, \mathrm{CDCl}_{3}\right) ;{ }^{1} \mathrm{H}$ NMR $(300$ $\left.\mathrm{MHz}, \mathrm{CDCl}_{3}\right): \delta 7.30-7.02(\mathrm{~m}, 7 \mathrm{H}), 6.63-6.56$ $(\mathrm{m}, 2 \mathrm{H}), 4.92\left(\mathrm{~d}, 1 \mathrm{H},{ }^{2} \mathrm{~J}_{\mathrm{H}-\mathrm{F}}=48.0 \mathrm{~Hz}\right), 4.32(\mathrm{~S}$, $2 \mathrm{H}), 4.10-3.95(\mathrm{~m}, 2 \mathrm{H}), 3.36(\mathrm{~d}, 1 \mathrm{H}, J=27$ $\mathrm{Hz}), 2.85-2.75(\mathrm{~m}, 2 \mathrm{H}), 2.07-1.91(\mathrm{~m}, 2 \mathrm{H})$, $1.15\left(\mathrm{t}, 3 \mathrm{H}, \mathrm{J}_{\mathrm{H}-\mathrm{H}}=6.0 \mathrm{~Hz}\right) .{ }^{19} \mathrm{~F}$ NMR $(282 \mathrm{MHz}$, $\left.\mathrm{CDCl}_{3}\right): \delta-196.0\left(\mathrm{dd},{ }^{2} \mathrm{~J}_{\mathrm{F}-\mathrm{H}}=48.0 \mathrm{~Hz},{ }^{3} \mathrm{~J}_{\mathrm{F}-\mathrm{H}}=\right.$ $25.4 \mathrm{~Hz}) .{ }^{13} \mathrm{C}\left\{{ }^{1} \mathrm{H}\right\}$ NMR $\left(75 \mathrm{MHz}, \mathrm{CDCl}_{3}\right): \delta$ $168.1\left(\mathrm{~d},{ }^{2} J_{\mathrm{C}-\mathrm{F}}=22.5 \mathrm{~Hz}\right), 149.2,140.7,137.3$, $130.5,128.5,128.4,126.2,118.4,115.1$, $114.9,89.8\left(\mathrm{~d},{ }^{1} J_{\mathrm{C}-\mathrm{F}}=187.5 \mathrm{~Hz}\right), 61.8,49.6(\mathrm{~d}$, $\left.{ }^{2} J_{C-F}=15.0 \mathrm{~Hz}\right), 33.2,32.9,14.0$. IR: 3468 , $3371,2925,1754,1607,1479,1216,1103$, 1022, 857, 747, 699, $454 \mathrm{~cm}^{-1}$. HRMS $\left(\mathrm{API}^{+}\right)$ $\mathrm{m} / \mathrm{z}:[\mathrm{M}+\mathrm{H}]^{+}$calcd for $\mathrm{C}_{19} \mathrm{H}_{23} \mathrm{FNO}_{2} \mathrm{~S}: 348.1434$, found: 348.1426 .

\section{(2S,3S)-ethyl}

2-fluoro-5-phenyl-3-(otolylthio)pentanoate (7). (Z)-ethyl 2-fluoro-5phenylpent-2-enoate $(0.3 \mathrm{mmol}, 66.6 \mathrm{mg})$, with $(\mathrm{DHQ})_{2} \mathrm{PYR}(0.03 \mathrm{mmol}, 26.4 \mathrm{mg})$ were stirred in neat 2-methylbenzenethiol $(1.5 \mathrm{mmol}, 186$ $\mathrm{mg}$ ) at $0{ }^{\circ} \mathrm{C}$ for 5 days according to the general procedure $\mathbf{b} .{ }^{19} \mathrm{~F}$ NMR of crude product showed $d r=95: 5$. The crude was purified by silica gel column chromatography (petroleum ether/EtOAc, from $15 / 1$ to $10 / 1$, v/v) affording two diastereoisomers in $91 \%$ yield (major: 78 $\mathrm{mg}$ as a colorless oil; major and minor mixture: $16 \mathrm{mg}$ ). $78 \%$ ee was obtained for the major diastereoisomer [determined by HPLC, IC column, Hept $/ i-\mathrm{PrOH}: 90 / 10$, flow $=1 \mathrm{~mL} / \mathrm{min}$, $\lambda=254 \mathrm{~nm}, \mathrm{t}$ (minor) $=5.793 \mathrm{~min}, \mathrm{t}$ (major) $=$ $6.470 \mathrm{~min}$.]. The racemic product has been obtained following the procedure a the same scale) in $91 \%$ yield (major: $68 \mathrm{mg}$ as a colorless oil; major and minor mixture: $26 \mathrm{mg}$ ) with $d r=$ 73:27.

$[\alpha]_{D}{ }^{20}=+29.4\left(\mathrm{c} 0.68, \mathrm{CDCl}_{3}\right) ;{ }^{1} \mathrm{H}$ NMR $(300$ $\left.\mathrm{MHz}, \mathrm{CDCl}_{3}\right): \delta 7.24-7.06(\mathrm{~m}, 9 \mathrm{H}), 4.98(\mathrm{~d}$, $\left.1 \mathrm{H},{ }^{2} J_{\mathrm{H}-\mathrm{F}}=48.0 \mathrm{~Hz}\right), 4.08-3.83(\mathrm{~m}, 2 \mathrm{H}), 3.57$ $-3.45(\mathrm{~m}, 1 \mathrm{H}), 2.84-2.75(\mathrm{~m}, 2 \mathrm{H}), 2.34(\mathrm{~S}$, $3 \mathrm{H}), 2.15-1.97(\mathrm{~m}, 2 \mathrm{H}), 1.10\left(\mathrm{t}, 3 \mathrm{H}, \mathrm{J}_{\mathrm{H}-\mathrm{H}}=9.0\right.$ $\mathrm{Hz}) .{ }^{19} \mathrm{~F} \mathrm{NMR}\left(282 \mathrm{MHz}, \mathrm{CDCl}_{3}\right): \delta-196.5$ (dd, $\left.{ }^{2} J_{F-H}=48.0 \mathrm{~Hz},{ }^{3} J_{F-H}=28.2 \mathrm{~Hz}\right) \cdot{ }^{13} \mathrm{C}\left\{{ }^{1} \mathrm{H}\right\}$ NMR (75 MHz, $\mathrm{CDCl}_{3}$ ): $\delta 167.8$ (d, ${ }^{2} \mathrm{~J}_{\mathrm{C}-\mathrm{F}}=22.5 \mathrm{~Hz}$ ), $140.6,140.1,133.3,132.3,130.4,128.5$, $128.5,127.4,126.5,126.2,90.0\left(\mathrm{~d},{ }^{1} J_{\mathrm{C}-\mathrm{F}}=\right.$ $187.5 \mathrm{~Hz}$ ), 61.7, $49.7\left(\mathrm{~d},{ }^{2} \mathrm{~J}_{\mathrm{C}-\mathrm{F}}=22.5 \mathrm{~Hz}\right), 33.5$, 33.2, 20.9, 13.9. IR: 2935, 1761, 1736, 1454, 1299, 1212, 1025, 747, 699, 558, $438 \mathrm{~cm}^{-1}$. HRMS $\left(\mathrm{API}^{+}\right) \quad \mathrm{m} / \mathrm{z}: \quad[\mathrm{M}+\mathrm{H}]^{+}$calcd for $\mathrm{C}_{20} \mathrm{H}_{24} \mathrm{FO}_{2} \mathrm{~S}: 347.1481$, found: 347.1480 .

(2S,3S)-ethyl 2-fluoro-5-phenyl-3-(mtolylthio)pentanoate (8). (Z)-ethyl 2-fluoro-5phenylpent-2-enoate $(0.3 \mathrm{mmol}, 66.6 \mathrm{mg})$, with $(\mathrm{DHQ})_{2} \mathrm{PYR}(0.03 \mathrm{mmol}, 26.4 \mathrm{mg})$ were stirred in neat 3-methylbenzenethiol $(1.5 \mathrm{mmol}, 186$ $\mathrm{mg}$ ) at $0{ }^{\circ} \mathrm{C}$ for 5 days according to the general procedure b. ${ }^{19} \mathrm{~F}$ NMR of crude product showed $d r=94: 6$. The crude was purified by silica gel column chromatography (petroleum ether/EtOAc, from 15/1 to $10 / 1, \mathrm{v} / \mathrm{v}$ ) affording two diastereoisomers in 95\% yield (major: 86 $\mathrm{mg}$ as a colorless oil; major and minor mixture: $13 \mathrm{mg}$ ). $80 \%$ ee was obtained for the major diastereoisomer [determined by HPLC, IC column, Hept $/ i$-PrOH: $90 / 10$, flow $=1 \mathrm{~mL} / \mathrm{min}$, $\lambda=254 \mathrm{~nm}, \mathrm{t}$ (minor) $=6.873 \mathrm{~min}, \mathrm{t}$ (major) $=$ $7.530 \mathrm{~min}$.]. The racemic product has been obtained following the procedure a (the same scale) in $84 \%$ yield (major: $66 \mathrm{mg}$ as a colorless oil; major and minor mixture: $21 \mathrm{mg}$ ) with $d r=$ 72:28.

$[\alpha]_{D}{ }^{20}=-18.8\left(\mathrm{c} 0.89, \mathrm{CDCl}_{3}\right) ;{ }^{1} \mathrm{H}$ NMR $(300$ $\left.\mathrm{MHz}, \mathrm{CDCl}_{3}\right): \delta 7.23-6.97(\mathrm{~m}, 9 \mathrm{H}), 4.97(\mathrm{~d}$, $\left.1 \mathrm{H},{ }^{2} J_{\mathrm{H}-\mathrm{F}}=48.0 \mathrm{~Hz}, J_{\mathrm{H}-\mathrm{H}} 3.0 \mathrm{~Hz}\right), 4.12-3.93$ $(\mathrm{m}, 2 \mathrm{H}), 3.47-3.34(\mathrm{~m}, 1 \mathrm{H}), 2.91-2.69(\mathrm{~m}$, $2 \mathrm{H}), 2.23(\mathrm{~S}, 3 \mathrm{H}), 2.06-1.89(\mathrm{~m}, 2 \mathrm{H}), 1.13(\mathrm{t}$, $\left.3 \mathrm{H}, \mathrm{J}_{\mathrm{H}-\mathrm{H}}=9.0 \mathrm{~Hz}\right) .{ }^{19} \mathrm{~F} \mathrm{NMR}\left(282 \mathrm{MHz}, \mathrm{CDCl}_{3}\right)$ : 
$\delta-196.8\left(\mathrm{dd},{ }^{2} J_{\mathrm{F}-\mathrm{H}}=48.0 \mathrm{~Hz},{ }^{3} J_{\mathrm{F}-\mathrm{H}}=28.2 \mathrm{~Hz}\right)$. ${ }^{13} \mathrm{C}\left\{{ }^{1} \mathrm{H}\right\}$ NMR $\left(75 \mathrm{MHz}, \mathrm{CDCl}_{3}\right): \delta 168.0\left(\mathrm{~d},{ }^{2} \mathrm{~J}_{\mathrm{C}}\right.$ $\mathrm{F}=22.5 \mathrm{~Hz}), 140.7,138.7,133.5,133.3,129.7$, $128.8,128.5,128.4,126.2,90.5\left(\mathrm{~d},{ }^{1} J_{\mathrm{C}-\mathrm{F}}=\right.$ $187.5 \mathrm{~Hz}), 61.7,50.4\left(\mathrm{~d},{ }^{2} \mathrm{~J}_{\mathrm{C}-\mathrm{F}}=22.5 \mathrm{~Hz}\right), 33.4$, 33.1, 21.3, 14.0. IR: 2933, 1762, 1737, 1454, 1299, 1212, 1025, 749, 698, 557, $432 \mathrm{~cm}^{-1}$. HRMS $\left(\mathrm{API}^{+}\right) \quad \mathrm{m} / \mathrm{z}: \quad[\mathrm{M}+\mathrm{H}]^{+}$calcd for $\mathrm{C}_{20} \mathrm{H}_{24} \mathrm{FO}_{2} \mathrm{~S}$ : 347.1481, found: 347.1479.

\section{(2S,3S)-ethyl}

2-fluoro-5-phenyl-3-(ptolylthio)pentanoate (9). (Z)-ethyl 2-fluoro-5phenylpent-2-enoate $(0.3 \mathrm{mmol}, 66.6 \mathrm{mg})$, with $(\mathrm{DHQ})_{2} \mathrm{PYR}(0.03 \mathrm{mmol}, 26.4 \mathrm{mg})$ were stirred in neat 4-methylbenzenethiol $(1.5 \mathrm{mmol}, 186$ $\mathrm{mg}$ ) at $0{ }^{\circ} \mathrm{C}$ for 5 days according to the general procedure $\mathbf{b} .{ }^{19} \mathrm{~F}$ NMR of crude product showed $d r=85: 15$. The crude was purified by silica gel column chromatography (petroleum ether/EtOAc, from $15 / 1$ to $10 / 1, v / v$ ) affording two diastereoisomers in $82 \%$ yield (major: 77 $\mathrm{mg}$ as a colorless oil; minor: $8 \mathrm{mg}$ as a colorless oil). $54 \%$ ee was obtained for the major diastereoisomer [determined by HPLC, IC column, Hept $/ i-\mathrm{PrOH}: 90 / 10$, flow $=1 \mathrm{~mL} / \mathrm{min}, \lambda$ $=254 \mathrm{~nm}, \mathrm{t}$ (minor) $=7.347 \mathrm{~min}, \mathrm{t}$ (major) $=$ $8.143 \mathrm{~min}$.]. The racemic product has been obtained following the procedure a the same scale) in $67 \%$ yield (major: $31 \mathrm{mg}$ as a colorless oil; minor: $9 \mathrm{mg}$ as a colorless oil; major and minor mixture: $30 \mathrm{mg}$ ) with $d r=74: 26$.

$[\alpha]_{D}{ }^{20}=-14.0$ (c $\left.0.84, \mathrm{CDCl}_{3}\right) ;{ }^{1} \mathrm{H}$ NMR $(300$ $\left.\mathrm{MHz}, \mathrm{CDCl}_{3}\right): \delta 7.28-7.00(\mathrm{~m}, 9 \mathrm{H}), 4.94(\mathrm{~d}$, $\left.1 \mathrm{H},{ }^{2} J_{\mathrm{H}-\mathrm{F}}=48.0 \mathrm{~Hz}\right), 4.13-3.97(\mathrm{~m}, 2 \mathrm{H}), 3.40$ $-3.25(\mathrm{~m}, 1 \mathrm{H}), 2.92-2.68(\mathrm{~m}, 2 \mathrm{H}), 2.24(\mathrm{~S}$, $3 \mathrm{H}), 2.03-1.91(\mathrm{~m}, 2 \mathrm{H}), 1.15\left(\mathrm{t}, 3 \mathrm{H}, J_{\mathrm{H}-\mathrm{H}}=6.0\right.$ $\mathrm{Hz}) .{ }^{19} \mathrm{~F} \mathrm{NMR}\left(282 \mathrm{MHz}, \mathrm{CDCl}_{3}\right): \delta-196.8$ (dd, $\left.{ }^{2} J_{\mathrm{F}-\mathrm{H}}=48.0 \mathrm{~Hz},{ }^{3} J_{\mathrm{F}-\mathrm{H}}=28.2 \mathrm{~Hz}\right) \cdot{ }^{13} \mathrm{C}\left\{{ }^{1} \mathrm{H}\right\} \mathrm{NMR}$ $\left(75 \mathrm{MHz}, \mathrm{CDCl}_{3}\right): \delta 168.0\left(\mathrm{~d},{ }^{2} J_{\mathrm{C}-\mathrm{F}}=22.5 \mathrm{~Hz}\right.$ ), $140.8,137.9,133.5,129.7,129.7,128.5$, 128.5, 126.2, $90.6\left(\mathrm{~d},{ }^{1} \mathrm{~J}_{\mathrm{C}-\mathrm{F}}=187.5 \mathrm{~Hz}\right), 61.7$, $50.7\left(\mathrm{~d},{ }^{2} \mathrm{~J}_{\mathrm{C}-\mathrm{F}}=15.0 \mathrm{~Hz}\right), 33.2,33.2,21.1,14.0$. IR: 2920, 1762, 1737, 1493, 1300, 1211, 1104, 1020, 811, 749, 699, 556, $496 \mathrm{~cm}^{-1}$. HRMS $\left(\mathrm{API}^{+}\right) \mathrm{m} / \mathrm{z}:[\mathrm{M}+\mathrm{H}]^{+}$calcd for $\mathrm{C}_{20} \mathrm{H}_{24} \mathrm{FO}_{2} \mathrm{~S}$ : 347.1481, found: 347.1481.

(2S,3S)-ethyl 2-fluoro-5-phenyl-3-(thiophen-2ylthio)pentanoate (10). (Z)-ethyl 2-fluoro-5phenylpent-2-enoate $(0.3 \mathrm{mmol}, 66.6 \mathrm{mg})$, with (DHQ) ${ }_{2}$ PYR (0.03 mmol, $26.4 \mathrm{mg}$ ) were stirred in neat thiophene-2-thiol ( $1.5 \mathrm{mmol}, 174 \mathrm{mg})$ at $0{ }^{\circ} \mathrm{C}$ for 5 days according to the general procedure $\mathbf{b} .{ }^{19} \mathrm{~F}$ NMR of crude product showed $d r=88: 12$. The crude was purified by silica gel column chromatography (petroleum ether/EtOAc, from 15/1 to 10/1, v/v) affording two diastereoisomers in $73 \%$ yield (major: 41 mg as a yellow oil; major and minor mixture: 33 $\mathrm{mg} \mathrm{).} 67 \%$ ee was obtained for the major diastereoisomer [determined by HPLC, IC column, Hept $/ i-\mathrm{PrOH}: 90 / 10$, flow $=1 \mathrm{~mL} / \mathrm{min}$, $\lambda=254 \mathrm{~nm}, \mathrm{t}($ minor $)=6.183 \mathrm{~min}, \mathrm{t}$ (major) $=$ $7.050 \mathrm{~min}$.]. The racemic product has been obtained following the procedure a (the same scale) in $83 \%$ yield (major: $60 \mathrm{mg}$ as a yellow oil; major and minor mixture: $24 \mathrm{mg}$ ) with $d r=$ 83:17.

$[\alpha]_{D}^{20}=-15.7$ (c $\left.0.69, \mathrm{CDCl}_{3}\right) ;{ }^{1} \mathrm{H}$ NMR $(300$ $\left.\mathrm{MHz}, \mathrm{CDCl}_{3}\right): \delta 7.33-7.31(\mathrm{~m}, 1 \mathrm{H}), 7.24-7.10$ $(\mathrm{m}, 6 \mathrm{H}), 6.92-6.89(\mathrm{~m}, 1 \mathrm{H}), 4.91\left(\mathrm{dd}, 1 \mathrm{H},{ }^{2} J_{\mathrm{H}}\right.$ $\left.\mathrm{F}=48.0 \mathrm{~Hz}, \mathrm{~J}_{\mathrm{H}-\mathrm{H}}=6.0 \mathrm{~Hz}\right), 4.20-4.08(\mathrm{~m}, 2 \mathrm{H})$, $3.21-3.14(\mathrm{~m}, 1 \mathrm{H}), 2.95-2.73(\mathrm{~m}, 2 \mathrm{H}), 1.99$ $-1.91(\mathrm{~m}, 2 \mathrm{H}), 1.21\left(\mathrm{t}, 3 \mathrm{H}, \mathrm{J}_{\mathrm{H}-\mathrm{H}}=6.0 \mathrm{~Hz}\right) .{ }^{19} \mathrm{~F}$ NMR (282 MHz, $\left.\mathrm{CDCl}_{3}\right): \delta-196.5\left(\mathrm{dd},{ }^{2} \mathrm{~J}_{\mathrm{F}-\mathrm{H}}=\right.$ $\left.48.0 \mathrm{~Hz},{ }^{3} \mathrm{~J}_{\mathrm{F}-\mathrm{H}}=25.4 \mathrm{~Hz}\right) \cdot{ }^{13} \mathrm{C}\left\{{ }^{1} \mathrm{H}\right\}$ NMR $(75$ $\left.\mathrm{MHz}, \mathrm{CDCl}_{3}\right): \delta 167.9\left(\mathrm{~d},{ }^{2} \mathrm{~J}_{\mathrm{C}-\mathrm{F}}=22.5 \mathrm{~Hz}\right.$ ), $140.7,136.5,130.8,130.6,128.6,128.5$, 127.6, 126.2, $90.4\left(\mathrm{~d},{ }^{1} \mathrm{~J}_{\mathrm{C}-\mathrm{F}}=195.0 \mathrm{~Hz}\right), 61.8$, $52.4\left(\mathrm{~d},{ }^{2} \mathrm{~J}_{\mathrm{C}-\mathrm{F}}=15.0 \mathrm{~Hz}\right), 33.1,32.5,32.4,14.1$. IR: 2940, 1760, 1737, 1216, 1023, 847, 749, 698, $493 \mathrm{~cm}^{-1}$. HRMS (API+) m/z: $[\mathrm{M}+\mathrm{H}]^{+}$calcd for $\mathrm{C}_{17} \mathrm{H}_{20} \mathrm{FO}_{2} \mathrm{~S}_{2}$ : 339.0889, found: 339.0899 .

(2S,3S)-ethyl 2-fluoro-3-(naphthalen-2-ylthio)5-phenylpentanoate (11). (Z)-ethyl 2-fluoro-5phenylpent-2-enoate $(0.3 \mathrm{mmol}, 66.6 \mathrm{mg})$, with (DHQ) ${ }_{2}$ PYR (0.03 mmol, $26.4 \mathrm{mg}$ ) were stirred in neat naphthalene-2-thiol $(1.5 \mathrm{mmol}, 240 \mathrm{mg})$ at $0{ }^{\circ} \mathrm{C}$ for 8 days according to the general procedure b. ${ }^{19} \mathrm{~F} \mathrm{NMR}$ of crude product showed $d r=73: 27$. The crude was purified by silica gel column chromatography (petroleum ether/EtOAc, from $15 / 1$ to $10 / 1, v / v$ ) affording two diastereoisomers in $31 \%$ yield (major: 27 $\mathrm{mg}$ as a colorless oil; major and minor mixture: $8 \mathrm{mg} \mathrm{).} \mathrm{29 \%} \mathrm{ee} \mathrm{was} \mathrm{obtained} \mathrm{for} \mathrm{the} \mathrm{major}$ diastereoisomer [determined by HPLC, IC column, Hept $/ i-\mathrm{PrOH}: 90 / 10$, flow $=1 \mathrm{~mL} / \mathrm{min}$, $\lambda=254 \mathrm{~nm}, \mathrm{t}$ (minor) $=7.777 \mathrm{~min}, \mathrm{t}$ (major) $=$ $8.900 \mathrm{~min}$.]. The racemic product has been obtained following the procedure a (the same 
scale) in $84 \%$ yield (major: $80 \mathrm{mg}$ as a colorless oil; major and minor mixture: $16 \mathrm{mg}$ ) with $d r=$ $80: 20$.

$[\alpha]_{D}^{20}=-7.7$ (c 0.57, $\left.\mathrm{CDCl}_{3}\right) ;{ }^{1} \mathrm{H}$ NMR $(300 \mathrm{MHz}$, $\left.\mathrm{CDCl}_{3}\right): \delta 7.80-7.65(\mathrm{~m}, 4 \mathrm{H}), 7.41-7.38(\mathrm{~m}$, $3 \mathrm{H}), 7.21-7.06(\mathrm{~m}, 5 \mathrm{H}), 5.01\left(\mathrm{~d}, 1 \mathrm{H},{ }^{2} \mathrm{~J}_{\mathrm{H}-\mathrm{F}}=\right.$ $48.0 \mathrm{~Hz}), 4.06-3.86(\mathrm{~m}, 2 \mathrm{H}), 3.60-3.46(\mathrm{~m}$, $1 \mathrm{H}), 2.95-2.72(\mathrm{~m}, 2 \mathrm{H}), 2.12-2.00(\mathrm{~m}, 2 \mathrm{H})$, $1.07\left(\mathrm{t}, 3 \mathrm{H}, J_{\mathrm{H}-\mathrm{H}}=6.0 \mathrm{~Hz}\right) .{ }^{19} \mathrm{~F} \mathrm{NMR}(282 \mathrm{MHz}$, $\left.\mathrm{CDCl}_{3}\right): \delta-196.8\left(\mathrm{dd},{ }^{2} J_{\mathrm{F}-\mathrm{H}}=48.0 \mathrm{~Hz},{ }^{3} J_{\mathrm{F}-\mathrm{H}}=\right.$ $28.2 \mathrm{~Hz}) \cdot{ }^{13} \mathrm{C}\left\{{ }^{1} \mathrm{H}\right\}$ NMR $\left(75 \mathrm{MHz}, \mathrm{CDCl}_{3}\right): \delta$ $167.9\left(\mathrm{~d},{ }^{2} \mathrm{~J}_{\mathrm{C}-\mathrm{F}}=22.5 \mathrm{~Hz}\right), 140.8,133.5,132.4$, $131.6,131.1,129.8,128.6,128.5,127.7$, $127.4,126.6,126.4,126.3,90.5\left(\mathrm{~d},{ }^{1} J_{\mathrm{C}-\mathrm{F}}=\right.$ $187.5 \mathrm{~Hz}$ ), 61.7, $50.3\left(\mathrm{~d},{ }^{2} \mathrm{~J}_{\mathrm{C}-\mathrm{F}}=22.5 \mathrm{~Hz}\right), 33.4$, 33.2, 14.0. IR: 2933, 1760, 1736, 1497, 1454, $1212,1103,1023,858,814,744,699,475 \mathrm{~cm}^{-}$ 1. HRMS $\left(\mathrm{API}^{+}\right) \mathrm{m} / \mathrm{z}:[\mathrm{M}+\mathrm{H}]^{+}$calcd for $\mathrm{C}_{23} \mathrm{H}_{24} \mathrm{FO}_{2} \mathrm{~S}: 383.1481$, found: 383.1477.

(2S,3S)-ethyl 3-((4-bromophenyl)thio)-2-fluoro5-(4-nitrophenyl)pentanoate (12). (Z)-ethyl 2fluoro-5-(4-nitrophenyl)pent-2-enoate $\quad(0.3$ mmol, $80.1 \mathrm{mg})$, 4-bromobenzenethiol (1.5 $\mathrm{mmol}, 283.5 \mathrm{mg})$ with (DHQ) ${ }_{2} \mathrm{PYR}(0.03 \mathrm{mmol}$, $26.4 \mathrm{mg}$ ) were stirred in toluene at $0{ }^{\circ} \mathrm{C}$ for 8 days according to the general procedure $\mathbf{b} .{ }^{19} \mathrm{~F}$ NMR of crude product showed $d r=80: 20$. The crude was purified by silica gel column chromatography (petroleum ether/EtOAc, from $15 / 1$ to $10 / 1, \mathrm{v} / \mathrm{v})$ affording two diastereoisomers in 77\% yield (major: $72 \mathrm{mg}$ as a yellow oil; major and minor mixture: $33 \mathrm{mg}$ ). $2 \%$ ee was obtained for the major diastereoisomer [determined by HPLC, IC column, Hept $/ i$-PrOH: 99/1, flow $=1 \mathrm{~mL} / \mathrm{min}, \lambda$ $=254 \mathrm{~nm}, \mathrm{t}($ minor $)=125.503 \mathrm{~min}, \mathrm{t}$ (major $)=$ $115.393 \mathrm{~min}$.]. The racemic product has been obtained following the procedure a the same scale) in $73 \%$ yield (major: $75 \mathrm{mg}$ as a yellow oil; major and minor mixture: $24 \mathrm{mg}$ ) with $d r=$ 82:18.

$[\alpha]_{D}{ }^{20}=-0.34$ (c $\left.0.87, \mathrm{CDCl}_{3}\right) ;{ }^{1} \mathrm{H}$ NMR $(300$ $\left.\mathrm{MHz}, \mathrm{CDCl}_{3}\right): \delta 8.07\left(\mathrm{~d}, 2 \mathrm{H}, \mathrm{J}_{\mathrm{H}-\mathrm{H}}=9.0 \mathrm{~Hz}\right), 7.37$ - $7.34(\mathrm{~m}, 2 \mathrm{H}), 7.27$ - $7.19(\mathrm{~m}, 4 \mathrm{H}), 4.98$ (dd, $\left.1 \mathrm{H},{ }^{2} J_{\mathrm{H}-\mathrm{F}}=48.0 \mathrm{~Hz}, J_{\mathrm{H}-\mathrm{H}}=3.0 \mathrm{~Hz}\right), 4.21-4.01$ $(\mathrm{m}, 2 \mathrm{H}), 3.43-3.28(\mathrm{~m}, 1 \mathrm{H}), 3.03-2.77(\mathrm{~m}$, $2 \mathrm{H}), 2.14-1.91(\mathrm{~m}, 2 \mathrm{H}), 1.19\left(\mathrm{t}, 3 \mathrm{H}, \mathrm{J}_{\mathrm{H}-\mathrm{H}}=6.0\right.$ $\mathrm{Hz}) \cdot{ }^{19} \mathrm{~F} \mathrm{NMR}\left(282 \mathrm{MHz}, \mathrm{CDCl}_{3}\right): \delta-196.4$ (dd, $\left.{ }^{2} J_{\mathrm{F}-\mathrm{H}}=48.0 \mathrm{~Hz},{ }^{3} J_{\mathrm{F}-\mathrm{H}}=28.2 \mathrm{~Hz}\right) \cdot{ }^{13} \mathrm{C}\left\{{ }^{1} \mathrm{H}\right\} \mathrm{NMR}$ $\left(75 \mathrm{MHz}, \mathrm{CDCl}_{3}\right): \delta 167.6\left(\mathrm{~d},{ }^{2} J_{\mathrm{C}-\mathrm{F}}=30.0 \mathrm{~Hz}\right)$,
$148.3,146.7,134.7,132.3,132.2,129.2$, 123.9, $122.4,90.5(\mathrm{~d}, \mathrm{C}-\mathrm{F},=187.5 \mathrm{~Hz}), 62.0$, $50.6\left(\mathrm{~d},{ }^{2} \mathrm{~J}_{\mathrm{C}-\mathrm{F}}=22.5 \mathrm{~Hz}\right), 33.0,32.9,14.1$. IR: 2940, 1759, 1737, 1599, 1516, 1343, 1068, 852, 817, 698, $479 \mathrm{~cm}^{-1}$. HRMS (API) m/z: [M] calcd for $\mathrm{C}_{19} \mathrm{H}_{19} \mathrm{BrFNO}_{4} \mathrm{~S}$ : 455.0202 , found: 455.0193 .

(2S,3S)-ethyl 2-fluoro-3-(naphthalen-2-ylthio)5-(4-nitrophenyl)pentanoate (13). (Z)-ethyl 2fluoro-5-(4-nitrophenyl)pent-2-enoate $\quad(0.3$ $\mathrm{mmol}, 80.1 \mathrm{mg})$, naphthalene-2-thiol (1.5 mmol, $240 \mathrm{mg}$ ) with (DHQ) $)_{2} \mathrm{PYR}(0.03 \mathrm{mmol}$, $26.4 \mathrm{mg}$ ) were stirred in toluene at $0{ }^{\circ} \mathrm{C}$ for 8 days according to the general procedure $\mathbf{b} .{ }^{19} \mathrm{~F}$ NMR of crude product showed $d r=84: 16$. The crude was purified by silica gel column chromatography (petroleum ether/EtOAc, from $10 / 1$ to $8 / 1, v / v$ ) affording two diastereoisomers in $55 \%$ yield (major: $47 \mathrm{mg}$ as a yellow solid; major and minor mixture: $23 \mathrm{mg}$ ). $8 \%$ ee was obtained for the major diastereoisomer [determined by HPLC, IC column, Hept $/ i-\mathrm{PrOH}$ : $90 / 10$, flow $=1 \mathrm{~mL} / \mathrm{min}, \lambda=254 \mathrm{~nm}, \mathrm{t}$ (minor) $=$ $35.240 \mathrm{~min}, \mathrm{t}$ (major) $=45.267 \mathrm{~min}$.]. The racemic product has been obtained following the procedure a (the same scale) in $75 \%$ yield (major: $71 \mathrm{mg}$ as a yellow solid; major and minor mixture: $25 \mathrm{mg}$ ) with $d r=87: 13$.

$[\alpha]_{D}{ }^{20}=-2.2\left(\mathrm{c} 0.68, \mathrm{CDCl}_{3}\right) ; \mathrm{Mp} 86-88^{\circ} \mathrm{C} ;{ }^{1} \mathrm{H}$ $\operatorname{NMR}\left(300 \mathrm{MHz}, \mathrm{CDCl}_{3}\right): \delta 8.00\left(\mathrm{~d}, 2 \mathrm{H}, \mathrm{J}_{\mathrm{H}-\mathrm{H}}=\right.$ $9.0 \mathrm{~Hz}), 7.84(\mathrm{~s}, 1 \mathrm{H}), 7.76-7.65(\mathrm{~m}, 3 \mathrm{H}), 7.45$ - $7.40(\mathrm{~m}, 3 \mathrm{H}), 7.20-7.18(\mathrm{~m}, 2 \mathrm{H}), 5.02(\mathrm{dd}$, $\left.1 \mathrm{H},{ }^{2} J_{\mathrm{H}-\mathrm{F}}=48.0 \mathrm{~Hz}, \mathrm{~J}_{\mathrm{H}-\mathrm{H}}=3.0 \mathrm{~Hz}\right), 4.16-3.93$ $(\mathrm{m}, 2 \mathrm{H}), 3.59-3.44(\mathrm{~m}, 1 \mathrm{H}), 3.07-2.82(\mathrm{~m}$, $2 \mathrm{H}), 2.19-1.97(\mathrm{~m}, 2 \mathrm{H}), 1.13\left(\mathrm{t}, 3 \mathrm{H}, J_{\mathrm{H}-\mathrm{H}}=6.0\right.$ $\mathrm{Hz}) .{ }^{19} \mathrm{~F}$ NMR $\left(282 \mathrm{MHz}, \mathrm{CDCl}_{3}\right): \delta-196.0$ (dd, $\left.{ }^{2} J_{\mathrm{F}-\mathrm{H}}=48.0 \mathrm{~Hz},{ }^{3} \mathrm{~J}_{\mathrm{F}-\mathrm{H}}=28.2 \mathrm{~Hz}\right) \cdot{ }^{13} \mathrm{C}\left\{{ }^{1} \mathrm{H}\right\} \mathrm{NMR}$ $\left(75 \mathrm{MHz}, \mathrm{CDCl}_{3}\right): \delta 167.7$ (d, ${ }^{2} \mathrm{~J}_{\mathrm{C}-\mathrm{F}}=30.0 \mathrm{~Hz}$ ), $148.5,146.6,133.5,132.6,131.9,130.6$, $129.8,129.8,129.3,128.8,127.7,127.4$, 126.9, 126.7, 123.8, 90.5 (d, $\left.{ }^{1} J_{\mathrm{C}-\mathrm{F}}=187.5 \mathrm{~Hz}\right)$, $61.9,50.4\left(\mathrm{~d},{ }^{2} \mathrm{~J}_{\mathrm{C}-\mathrm{F}}=22.5 \mathrm{~Hz}\right), 33.1,33.0,14.0$. IR: 2925, 1764, 1490, 1222, 1109, 1027, 830, $523,468 \mathrm{~cm}^{-1}$. HRMS (API) m/z: [M] ${ }^{+}$calcd for $\mathrm{C}_{23} \mathrm{H}_{22} \mathrm{FNO}_{4} \mathrm{~S}: 427.1254$, found: 427.1254 .

(2S,3S)-ethyl 3-cyclohexyl-2-fluoro-3(phenylthio)propanoate (14). (Z)-ethyl 3cyclohexyl-2-fluoroacrylate $(0.3 \mathrm{mmol}, 60.0$ $\mathrm{mg})$, with $(\mathrm{DHQ})_{2} \mathrm{PYR}(0.03 \mathrm{mmol}, 26.4 \mathrm{mg})$ 
were stirred in neat benzenethiol $(1.5 \mathrm{mmol}$, $165 \mathrm{mg}$ ) at $0{ }^{\circ} \mathrm{C}$ for 5 days according to the general procedure $\mathbf{b} .{ }^{19} \mathrm{~F}$ NMR of crude product showed $d r=100: 0$. The crude was purified by silica gel column chromatography (petroleum ether/EtOAc, from $15 / 1$ to $10 / 1, \mathrm{v} / \mathrm{v}$ ) affording an only diastereoisomer in $34 \%$ yield $(32 \mathrm{mg}$ as a colorless oil). $65 \%$ ee was obtained for it [determined by HPLC, IC column, Hept $/ i-\mathrm{PrOH}$ : $90 / 10$, flow $=1 \mathrm{~mL} / \mathrm{min}, \lambda=254 \mathrm{~nm}, \mathrm{t}$ (minor) $=$ $6.607 \mathrm{~min}, \mathrm{t}$ (major) $=7.450 \mathrm{~min}$.]. The racemic product has been obtained following the procedure a (the same scale) in $86 \%$ yield (major: $61 \mathrm{mg}$ as a colorless oil; major and minor mixture: $19 \mathrm{mg}$ ) with $d r=82: 18$.

$[\alpha]_{D}{ }^{20}=-5.6$ (c 0.32, $\left.\mathrm{CDCl}_{3}\right) ;{ }^{1} \mathrm{H}$ NMR $(300 \mathrm{MHz}$, $\left.\mathrm{CDCl}_{3}\right)$ : $\delta 7.39-7.36(\mathrm{~m}, 2 \mathrm{H}), 7.22-7.13(\mathrm{~m}$, $3 \mathrm{H}), 5.20\left(\mathrm{dd}, 1 \mathrm{H},{ }^{2} J_{\mathrm{H}-\mathrm{F}}=48.0 \mathrm{~Hz}, J_{\mathrm{H}-\mathrm{H}}=3.0\right.$ $\mathrm{Hz}), 4.10-3.81(\mathrm{~m}, 2 \mathrm{H}), 3.34-3.19(\mathrm{~m}, 1 \mathrm{H})$, $2.14\left(\mathrm{~d}, 1 \mathrm{H}, J_{\mathrm{H}-\mathrm{H}}=15.0 \mathrm{~Hz}\right), 1.84-1.53(\mathrm{~m}$, $5 \mathrm{H}), 1.21-1.13(\mathrm{~m}, 5 \mathrm{H}), 1.07\left(\mathrm{t}, 3 \mathrm{H}, J_{\mathrm{H}-\mathrm{H}}=6.0\right.$ $\mathrm{Hz}) .{ }^{19} \mathrm{~F} \mathrm{NMR}\left(282 \mathrm{MHz}, \mathrm{CDCl}_{3}\right): \delta-197.9$ (dd, $\left.{ }^{2} J_{F-H}=48.0 \mathrm{~Hz},{ }^{3} J_{F-H}=36.7 \mathrm{~Hz}\right) \cdot{ }^{13} \mathrm{C}\left\{{ }^{1} \mathrm{H}\right\}$ NMR $\left(75 \mathrm{MHz}, \mathrm{CDCl}_{3}\right): \delta 168.6\left(\mathrm{~d},{ }^{2} J_{\mathrm{C}-\mathrm{F}}=30.0 \mathrm{~Hz}\right)$, $135.7,132.0,128.9,127.1,89.3\left(\mathrm{~d},{ }^{1} J_{\mathrm{C}-\mathrm{F}}=\right.$ $187.5 \mathrm{~Hz}), 61.6,57.9\left(\mathrm{~d},{ }^{2} J_{\mathrm{C}-\mathrm{F}}=15.0 \mathrm{~Hz}\right), 40.7$, 31.1, 30.8, 26.2, 26.1, 13.9. IR: 2925, 1763, 1735, 1440, 1214, 1102, 1024, 746, 691, 537, $481 \mathrm{~cm}^{-1}$. HRMS $\left(\mathrm{API}^{+}\right) \mathrm{m} / \mathrm{z}:[\mathrm{M}+\mathrm{H}]^{+}$calcd for $\mathrm{C}_{17} \mathrm{H}_{24} \mathrm{FO}_{2} \mathrm{~S}: 311.1481$, found: 311.1491.

(2S,3S)-ethyl 3-((4-bromophenyl)thio)-3cyclohexyl-2-fluoropropanoate (15). (Z)-ethyl 3-cyclohexyl-2-fluoroacrylate $(0.3 \mathrm{mmol}, 60.0$ $\mathrm{mg}$ ), with (DHQ) ${ }_{2} \mathrm{PYR}(0.03 \mathrm{mmol}, 26.4 \mathrm{mg})$ were stirred in neat 4-bromobenzenethiol (1.5 $\mathrm{mmol}, 283.5 \mathrm{mg}$ ) at $0{ }^{\circ} \mathrm{C}$ for 8 days according to the general procedure $\mathbf{b} .{ }^{19} \mathrm{~F}$ NMR of crude product showed $d r=69: 31$. The crude was purified by silica gel column chromatography (petroleum ether/EtOAc, from $10 / 1$ to $8 / 1$, v/v) affording two diastereoisomers in $38 \%$ yield (major: $24 \mathrm{mg}$ as a white solid; major and minor mixture: $20 \mathrm{mg}$ ). $11 \%$ ee was obtained for the major diastereoisomer [determined by HPLC, IC column, Hept $/ i-\mathrm{PrOH}$ : 90/10, flow $=1$ $\mathrm{mL} / \mathrm{min}, \lambda=254 \mathrm{~nm}, \mathrm{t}$ (minor) $=5.987 \mathrm{~min}, \mathrm{t}$ (major) $=6.587 \mathrm{~min}$.]. The racemic product has been obtained following the procedure a (the same scale) in $76 \%$ yield (major: $62 \mathrm{mg}$ as a white solid; major and minor mixture: $24 \mathrm{mg}$ ) with $d r=71: 29$.

$[\alpha]_{D}^{20}=+2.5\left(\right.$ c 1.02, $\left.\mathrm{CDCl}_{3}\right) ; \mathrm{Mp} 31-33^{\circ} \mathrm{C} ;{ }^{1} \mathrm{H}$ $\operatorname{NMR}\left(300 \mathrm{MHz}, \mathrm{CDCl}_{3}\right): \delta 7.33-7.23(\mathrm{~m}, 4 \mathrm{H})$, $5.20\left(\mathrm{dd}, 1 \mathrm{H},{ }^{2} J_{\mathrm{H}-\mathrm{F}}=48.0 \mathrm{~Hz}, J_{\mathrm{H}-\mathrm{H}}=3.0 \mathrm{~Hz}\right)$, $4.15-3.90(\mathrm{~m}, 2 \mathrm{H}), 3.28-3.13(\mathrm{~m}, 1 \mathrm{H}), 2.09$ $\left(\mathrm{d}, 1 \mathrm{H}, J_{\mathrm{H}-\mathrm{H}}=12.0 \mathrm{~Hz}\right), 1.82-1.59(\mathrm{~m}, 5 \mathrm{H})$, $1.18-0.98(\mathrm{~m}, 8 \mathrm{H}) .{ }^{19} \mathrm{~F}$ NMR $(282 \mathrm{MHz}$, $\left.\mathrm{CDCl}_{3}\right): \delta-197.7\left(\mathrm{dd},{ }^{2} J_{\mathrm{F}-\mathrm{H}}=48.0 \mathrm{~Hz},{ }^{3} J_{\mathrm{F}-\mathrm{H}}=\right.$ $33.8 \mathrm{~Hz}) .{ }^{13} \mathrm{C}\left\{{ }^{1} \mathrm{H}\right\} \mathrm{NMR}\left(75 \mathrm{MHz}, \mathrm{CDCl}_{3}\right): \delta$ $168.6\left(\mathrm{~d},{ }^{2} J_{\mathrm{C}-\mathrm{F}}=30.0 \mathrm{~Hz}\right), 134.7,133.7,121.3$, $89.3\left(\mathrm{~d},{ }^{1} J_{\mathrm{C}-\mathrm{F}}=195.0 \mathrm{~Hz}\right), 61.8,58.0\left(\mathrm{~d},{ }^{2} J_{\mathrm{C}-\mathrm{F}}=\right.$ $22.5 \mathrm{~Hz}$ ), 40.5, 31.0, 30.7, 26.1, 26.1, 14.0. IR: 2920, 1753, 1472, 1299, 1216, 817, 595, 466, $483 \mathrm{~cm}^{-1}$. HRMS $\left(\mathrm{API}^{+}\right) \mathrm{m} / \mathrm{z}$ : $[\mathrm{M}+\mathrm{H}]^{+}$calcd for $\mathrm{C}_{17} \mathrm{H}_{23} \mathrm{BrFO}_{2} \mathrm{~S}: 389.0586$, found: 389.0596 .

(2S,3S)-ethyl 3-cyclohexyl-2-fluoro-3-((4methoxyphenyl)thio)propanoate (16). (Z)-ethyl 3-cyclohexyl-2-fluoroacrylate $(0.3 \mathrm{mmol}, 60.0$ $\mathrm{mg})$, with (DHQ) ${ }_{2}$ PYR $(0.03 \mathrm{mmol}, 26.4 \mathrm{mg}$ ) were stirred in neat 4-methoxybenzenethiol $(1.5 \mathrm{mmol}, 210 \mathrm{mg})$ at $0^{\circ} \mathrm{C}$ for 5 days according to the general procedure $\mathbf{b} .{ }^{19} \mathrm{~F}$ NMR of crude product showed $d r=80: 20$. The crude was purified by silica gel column chromatography (petroleum ether/EtOAc, from 20/1 to 15/1, v/v) affording two diastereoisomers in $77 \%$ yield (major: $60 \mathrm{mg}$ as a white solid; major and minor mixture: $19 \mathrm{mg}$ ). $32 \%$ ee was obtained for the major diastereoisomer [determined by HPLC, IC column, Hept $/ i-\mathrm{PrOH}: 90 / 10$, flow $=1$ $\mathrm{mL} / \mathrm{min}, \lambda=254 \mathrm{~nm}, \mathrm{t}$ (minor) $=8.557 \mathrm{~min}, \mathrm{t}$ (major) $=10.183 \mathrm{~min}$.]. The racemic product has been obtained following the procedure a (the same scale) in 65\% yield (major: $47 \mathrm{mg}$ as a white solid; major and minor mixture: $19 \mathrm{mg}$ ) with $d r=78: 22$.

$[\alpha]_{D}{ }^{20}=-5.4\left(\right.$ c $\left.0.61, \mathrm{CDCl}_{3}\right) ; \mathrm{Mp} 57-58{ }^{\circ} \mathrm{C} ;{ }^{1} \mathrm{H}$ $\operatorname{NMR}\left(300 \mathrm{MHz}, \mathrm{CDCl}_{3}\right): \delta 7.35-7.33(\mathrm{~m}, 2 \mathrm{H})$, $6.76-6.73(\mathrm{~m}, 2 \mathrm{H}), 5.17\left(\mathrm{dd}, 1 \mathrm{H},{ }^{2} \mathrm{~J}_{\mathrm{H}-\mathrm{F}}=48.0\right.$ $\left.\mathrm{Hz}, J_{\mathrm{H}-\mathrm{H}}=2.4 \mathrm{~Hz}\right), 4.17-3.91(\mathrm{~m}, 2 \mathrm{H}), 3.72(\mathrm{~s}$, $3 \mathrm{H}), 3.14-3.00(\mathrm{~m}, 1 \mathrm{H}), 2.15\left(\mathrm{~d}, 1 \mathrm{H}, \mathrm{J}_{\mathrm{H}-\mathrm{H}}=6.0\right.$ $\mathrm{Hz}), 1.81-1.58(\mathrm{~m}, 5 \mathrm{H}), 1.18-1.01(\mathrm{~m}, 8 \mathrm{H})$. ${ }^{19} \mathrm{~F} \mathrm{NMR}\left(282 \mathrm{MHz}, \mathrm{CDCl}_{3}\right): \delta-197.9\left(\mathrm{dd},{ }^{2} J_{\mathrm{F}-\mathrm{H}}\right.$ $\left.=48.0 \mathrm{~Hz},{ }^{3} J_{\mathrm{F}-\mathrm{H}}=36.7 \mathrm{~Hz}\right) \cdot{ }^{13} \mathrm{C}\left\{{ }^{1} \mathrm{H}\right\}$ NMR $(75$ $\left.\mathrm{MHz}, \mathrm{CDCl}_{3}\right): \delta 168.8\left(\mathrm{~d},{ }^{2} J_{\mathrm{C}-\mathrm{F}}=22.5 \mathrm{~Hz}\right)$, $159.4,135.2,125.5,114.4,89.4\left(\mathrm{~d},{ }^{1} \mathrm{~J}_{\mathrm{C}-\mathrm{F}}=\right.$ $187.5 \mathrm{~Hz}), 61.6,58.7\left(\mathrm{~d},{ }^{2} \mathrm{~J}_{\mathrm{C}-\mathrm{F}}=15.0 \mathrm{~Hz}\right), 55.3$, 40.2, 31.1, 30.7, 26.2, 26.2, 14.0. IR: 2926, $1763,1590,1490,1222,1026,830,594,522$, 
$473 \mathrm{~cm}^{-1}$. HRMS $\left(\mathrm{API}^{+}\right) \mathrm{m} / \mathrm{z}:[\mathrm{M}+\mathrm{H}]^{+}$calcd for $\mathrm{C}_{18} \mathrm{H}_{26} \mathrm{FO}_{3} \mathrm{~S}$ : 341.1587, found: 341.1581.

(2S,3S)-ethyl 3-cyclohexyl-2-fluoro-3(thiophen-2-ylthio)propanoate (17). (Z)-ethyl 3cyclohexyl-2-fluoroacrylate $(0.3 \mathrm{mmol}, 60.0$ $\mathrm{mg})$, with (DHQ) ${ }_{2} \mathrm{PYR}(0.03 \mathrm{mmol}, 26.4 \mathrm{mg})$ were stirred in neat thiophene-2-thiol (1.5 $\mathrm{mmol}, 174 \mathrm{mg}$ ) at $0{ }^{\circ} \mathrm{C}$ for 5 days according to the general procedure b. ${ }^{19} \mathrm{~F}$ NMR of crude product showed $d r=100: 0$. The crude was purified by silica gel column chromatography (petroleum ether/EtOAc, from 15/1 to 10/1, v/v) affording an only diastereoisomer in $32 \%$ yield (30 mg as a yellow oil). 59\% ee was obtained for it [determined by HPLC, IC column, Hept $/ i$ PrOH: 90/10, flow $=1 \mathrm{~mL} / \mathrm{min}, \lambda=254 \mathrm{~nm}, \mathrm{t}$ (minor) $=6.583 \mathrm{~min}, \mathrm{t}$ (major) $=8.433 \mathrm{~min}$.] The racemic product has been obtained following the procedure a (the same scale) in $43 \%$ yield (major: $30 \mathrm{mg}$ as a yellow oil; major and minor mixture: $11 \mathrm{mg}$ ) with $d r=82: 18$.

$[\alpha]_{\mathrm{D}}{ }^{20}=-8.3\left(\mathrm{c} 0.06, \mathrm{CDCl}_{3}\right) ;{ }^{1} \mathrm{H} \mathrm{NMR}(300 \mathrm{MHz}$, $\left.\mathrm{CDCl}_{3}\right)$ : $\delta 7.29-7.26(\mathrm{~m}, 1 \mathrm{H}), 7.09-7.08(\mathrm{~m}$, $1 \mathrm{H}), 6.89-6.86(\mathrm{~m}, 1 \mathrm{H}), 5.17\left(\mathrm{dd}, 1 \mathrm{H},{ }^{2} J_{\mathrm{H}-\mathrm{F}}=\right.$ $\left.48.0 \mathrm{~Hz}, J_{\mathrm{H}-\mathrm{H}}=3.0 \mathrm{~Hz}\right), 4.26-4.02(\mathrm{~m}, 2 \mathrm{H})$, $3.11-2.96(\mathrm{~m}, 1 \mathrm{H}), 2.19\left(\mathrm{~d}, 1 \mathrm{H}, J_{\mathrm{H}-\mathrm{H}}=12.0\right.$ $\mathrm{Hz}), 1.83-1.63(\mathrm{~m}, 5 \mathrm{H}), 1.22-1.05(\mathrm{~m}, 8 \mathrm{H})$. ${ }^{19} \mathrm{~F}$ NMR $\left(282 \mathrm{MHz}, \mathrm{CDCl}_{3}\right): \delta-198.3\left(\mathrm{dd},{ }^{2} J_{\mathrm{F}-\mathrm{H}}\right.$ $\left.=48.0 \mathrm{~Hz},{ }^{3} J_{\mathrm{F}-\mathrm{H}}=33.8 \mathrm{~Hz}\right) \cdot{ }^{13} \mathrm{C}\left\{{ }^{1} \mathrm{H}\right\} \operatorname{NMR}(75$ $\left.\mathrm{MHz}, \mathrm{CDCl}_{3}\right): \delta 168.5\left(\mathrm{~d},{ }^{2} \mathrm{~J}_{\mathrm{C}-\mathrm{F}}=22.5 \mathrm{~Hz}\right)$, 135.4, 133.2, 130.0, 127.3, $89.1\left(\mathrm{~d},{ }^{1} \mathrm{~J}_{\mathrm{C}-\mathrm{F}}=\right.$ $195.0 \mathrm{~Hz}), 61.7,60.4\left(\mathrm{~d},{ }^{2} \mathrm{~J}_{\mathrm{C}-\mathrm{F}}=15.0 \mathrm{~Hz}\right), 39.8$, $31.1,30.5,26.2,26.1$, 14.1. IR: 2925, 1762, 1736, 1448, 1216, 1024, 847, 700, 533, 495 $\mathrm{cm}^{-1}$. HRMS $\left(\mathrm{API}^{+}\right) \mathrm{m} / \mathrm{z}$ : $[\mathrm{M}+\mathrm{H}]^{+}$calcd for $\mathrm{C}_{15} \mathrm{H}_{22} \mathrm{FO}_{2} \mathrm{~S}_{2}: 317.1045$, found: 317.1051.

\section{(2S,3S)-ethyl 3-((2-aminophenyl)thio)-3-} cyclohexyl-2-fluoropropanoate (18). (Z)-ethyl 3-cyclohexyl-2-fluoroacrylate $(0.3 \mathrm{mmol}, 60.0$ $\mathrm{mg})$, with (DHQ) ${ }_{2} \mathrm{PYR}(0.03 \mathrm{mmol}, 26.4 \mathrm{mg})$ were stirred in neat 2-aminobenzenethiol (1.5 $\mathrm{mmol}, 188.0 \mathrm{mg}$ ) at room temperature for 8 days according to the general procedure $\mathbf{b} .{ }^{19} \mathrm{~F}$ NMR of crude product showed $d r=90: 10$. The crude was purified by silica gel column chromatography (petroleum ether/EtOAc, from $10 / 1$ to $8 / 1, v / v$ ) affording two diastereoisomers in $55 \%$ yield (major: $45 \mathrm{mg}$ as a yellow solid, major and minor mixture: $9 \mathrm{mg}$ ). $87 \%$ ee was obtained for the major diastereoisomer [determined by HPLC, IC column, Hept/ $i-\mathrm{PrOH}$ : $90 / 10$, flow $=1 \mathrm{~mL} / \mathrm{min}, \lambda=254 \mathrm{~nm}, \mathrm{t}$ (minor) $=$ $16.160 \mathrm{~min}, \mathrm{t}$ (major) $=22.437 \mathrm{~min}$.]. The reaction performed on $1 \mathrm{mmol}$ of $(Z)$-ethyl 3cyclohexyl-2-fluoroacrylate led to $51 \%$ of isolated yield (major: $132 \mathrm{mg}$, major and minor mixture: $34 \mathrm{mg}$ ), dr:91:9 and ee: $85 \%$ (major diastereoisomer). The racemic product has been obtained following the procedure a (the same scale) in $75 \%$ yield (major: $38 \mathrm{mg}$ as a yellow solid; minor: $11 \mathrm{mg}$ as a yellow solid; major and minor mixture: $19 \mathrm{mg}$ ) with $d r=$ 80:20.

$[\alpha]_{D}^{20}=+24.1\left(\right.$ c $\left.0.22, \mathrm{CDCl}_{3}\right) ; \mathrm{Mp} 112-113$ ${ }^{\circ} \mathrm{C} ;{ }^{1} \mathrm{H}$ NMR $\left(300 \mathrm{MHz}, \mathrm{CDCl}_{3}\right): \delta 7.28(\mathrm{~d}, 1 \mathrm{H}$, $\left.J_{\mathrm{H}-\mathrm{H}}=6.0 \mathrm{~Hz}\right), 7.03-6.98(\mathrm{~m}, 1 \mathrm{H}), 6.61-6.56$ $(\mathrm{m}, 2 \mathrm{H}), 5.09\left(\mathrm{dd}, 1 \mathrm{H},{ }^{2} J_{\mathrm{H}-\mathrm{F}}=48.0 \mathrm{~Hz}, J_{\mathrm{H}-\mathrm{H}}=3.0\right.$ $\mathrm{Hz}), 4.29$ (s, 2H), $4.05-3.94(\mathrm{~m}, 1 \mathrm{H}), 3.80-$ $3.69(\mathrm{~m}, 1 \mathrm{H}), 3.35-3.21(\mathrm{~m}, 1 \mathrm{H}), 2.02-1.88$ $(\mathrm{m}, 2 \mathrm{H}), 1.74-1.61(\mathrm{~m}, 4 \mathrm{H}), 1.26-1.04(\mathrm{~m}$, 8H). ${ }^{19} \mathrm{~F} \mathrm{NMR}\left(282 \mathrm{MHz}, \mathrm{CDCl}_{3}\right): \delta-196.1$ (dd, $\left.{ }^{2} J_{F-H}=48.0 \mathrm{~Hz},{ }^{3} J_{F-H}=33.8 \mathrm{~Hz}\right) \cdot{ }^{13} \mathrm{C}\left\{{ }^{1} \mathrm{H}\right\} \mathrm{NMR}$ $\left(75 \mathrm{MHz}, \mathrm{CDCl}_{3}\right): \delta 168.7\left(\mathrm{~d},{ }^{2} \mathrm{~J}_{\mathrm{C}-\mathrm{F}}=30.0 \mathrm{~Hz}\right.$ ), 148.7, 136.5, 129.8, 118.4, 116.7, 115.0, 88.7 $\left(\mathrm{d},{ }^{1} J_{\mathrm{C}-\mathrm{F}}=187.5 \mathrm{~Hz}\right), 61.6,56.7\left(\mathrm{~d},{ }^{2} \mathrm{~J}_{\mathrm{C}-\mathrm{F}}=15.0\right.$ $\mathrm{Hz}), 40.9,31.0,30.5,30.5,26.4,26.3,26.2$, 13.9. IR: $3445,3351,2925,1748,1613,1480$, 1233, 737, 602, $454 \mathrm{~cm}^{-1}$. HRMS (API+) m/z: $[\mathrm{M}+\mathrm{H}]^{+}$calcd for $\mathrm{C}_{17} \mathrm{H}_{25} \mathrm{FNO}_{2} \mathrm{~S}$ : 326.1590, found: 326.1594.

(2S,3S)-ethyl 3-cyclohexyl-2-fluoro-3-(mtolylthio)propanoate (19). (Z)-ethyl 3cyclohexyl-2-fluoroacrylate $(0.3 \mathrm{mmol}, 60.0$ $\mathrm{mg}$ ), with (DHQ) ${ }_{2} \mathrm{PYR}(0.03 \mathrm{mmol}, 26.4 \mathrm{mg})$ were stirred in neat 3-methylbenzenethiol (1.5 $\mathrm{mmol}, 186.3 \mathrm{mg}$ ) at $0{ }^{\circ} \mathrm{C}$ for 5 days according to the general procedure b. ${ }^{19} \mathrm{~F}$ NMR of crude product showed $d r=81: 19$. The crude was purified by silica gel column chromatography (petroleum ether/EtOAc, from 15/1 to 10/1, v/v) affording two diastereoisomers in $44 \%$ yield (major: $35 \mathrm{mg}$ as a colorless oil, major and minor mixture: $8 \mathrm{mg}$ ). $26 \%$ ee was obtained for the major diastereoisomer [determined by HPLC, IC column, Hept/i-PrOH: 90/10, flow $=1$ $\mathrm{mL} / \mathrm{min}, \lambda=254 \mathrm{~nm}, \mathrm{t}$ (minor) $=7.013 \mathrm{~min}, \mathrm{t}$ (major) $=7.820 \mathrm{~min}$.]. The racemic product has been obtained following the procedure a (the same scale) in $67 \%$ yield (major: $54 \mathrm{mg}$ as a 
colorless oil; major and minor mixture: $11 \mathrm{mg}$ ) with $d r=80: 20$.

$[\alpha]_{D}{ }^{20}=-20.3$ (c $\left.0.10, \mathrm{CDCl}_{3}\right) ;{ }^{1} \mathrm{H}$ NMR $(300$ $\left.\mathrm{MHz}, \mathrm{CDCl}_{3}\right)$ : $\delta 7.19-7.15(\mathrm{~m}, 2 \mathrm{H}), 7.11-7.05$ $(\mathrm{m}, 1 \mathrm{H}), 6.96-6.93(\mathrm{~m}, 1 \mathrm{H}), 5.19\left(\mathrm{dd}, 1 \mathrm{H},{ }^{2} \mathrm{~J}_{\mathrm{H}}\right.$ $\left.\mathrm{F}=51.0 \mathrm{~Hz}, J_{\mathrm{H}-\mathrm{H}}=3.0 \mathrm{~Hz}\right), 4.11-3.81(\mathrm{~m}, 2 \mathrm{H})$, $3.34-3.19(\mathrm{~m}, 1 \mathrm{H}), 2.24(\mathrm{~s}, 3 \mathrm{H}), 2.13(\mathrm{~d}, 1 \mathrm{H}$, $\left.J_{H-H}=12.0 \mathrm{~Hz}\right), 1.71-1.60(\mathrm{~m}, 5 \mathrm{H}), 1.20-1.01$ $(\mathrm{m}, 8 \mathrm{H}) .{ }^{19} \mathrm{~F}$ NMR $\left(282 \mathrm{MHz}, \mathrm{CDCl}_{3}\right): \delta-197.7$ (dd, $\left.{ }^{2} J_{F-H}=51.0 \mathrm{~Hz},{ }^{3} J_{F-H}=33.8 \mathrm{~Hz}\right) .{ }^{13} \mathrm{C}\left\{{ }^{1} \mathrm{H}\right\}$ NMR $\left(75 \mathrm{MHz}, \mathrm{CDCl}_{3}\right): \delta 168.6\left(\mathrm{~d},{ }^{2} \mathrm{~J}_{\mathrm{C}-\mathrm{F}}=22.5\right.$ $\mathrm{Hz}), 138.6,135.5,132.4,128.9,128.7,127.9$, $89.3\left(\mathrm{~d},{ }^{1} \mathrm{~J}_{\mathrm{C}-\mathrm{F}}=187.5 \mathrm{~Hz}\right), 61.6,57.8\left(\mathrm{~d},{ }^{2} \mathrm{~J}_{\mathrm{C}-\mathrm{F}}=\right.$ $22.5 \mathrm{~Hz}), 40.8,31.1,30.8,26.2,26.1,21.3$, 13.9. IR: 2925, 1764, 1735, 1600, 1449, 1213, 1102, 1025, 856, 776, 690, 541, $436 \mathrm{~cm}^{-1}$. HRMS $\left(\mathrm{API}^{+}\right) \quad \mathrm{m} / \mathrm{z}: \quad[\mathrm{M}+\mathrm{H}]^{+}$calcd for $\mathrm{C}_{18} \mathrm{H}_{26} \mathrm{FO}_{2} \mathrm{~S}$ : 325.1638, found: 325.1647.

\section{(2S,3S)-methyl}

\section{(phenylthio)propanoate}

2-fluoro-3-phenyl-3fluoro-3-phenylacrylate $(0.3 \mathrm{mmol}, 54.4 \mathrm{mg})$ with (DHQ) ${ }_{2} P Y R(0.03 \mathrm{mmol}, 26.4 \mathrm{mg}$ ) were stirred in neat benzenethiol $(1.5 \mathrm{mmol}, 165.0$ $\mathrm{mg}$ ) at $0{ }^{\circ} \mathrm{C}$ for 8 days according to the general procedure b. ${ }^{19} \mathrm{~F}$ NMR of crude product showed $d r=88: 12$. The crude was purified by silica gel column chromatography (petroleum ether/EtOAc, from $15 / 1$ to $10 / 1, \mathrm{v} / \mathrm{v}$ ) affording two diastereoisomers in 54\% yield (major: 40 $\mathrm{mg}$ as a white solid, major and minor mixture: $7 \mathrm{mg}$ ). $63 \%$ ee was obtained for the major diastereoisomer [determined by HPLC, IC column, Hept $/ i$-PrOH: $90 / 10$, flow $=1 \mathrm{~mL} / \mathrm{min}, \lambda$ $=254 \mathrm{~nm}, \mathrm{t}$ (minor) $=6.530 \mathrm{~min}, \mathrm{t}$ (major) $=$ $7.053 \mathrm{~min}$.]. The racemic product has been obtained following the procedure a (the same scale) in $61 \%$ yield (major: $42 \mathrm{mg}$ as a white solid, major and minor mixture: $11 \mathrm{mg}$ ) with $d r$ $=83: 17$.

$[\alpha]_{\mathrm{D}}^{20}=+101.4\left(\mathrm{c} 0.67, \mathrm{CDCl}_{3}\right) ; \mathrm{Mp} 52-53^{\circ} \mathrm{C}$; ${ }^{1} \mathrm{H}$ NMR $\left(300 \mathrm{MHz}, \mathrm{CDCl}_{3}\right): \delta 7.31-7.22(\mathrm{~m}$, $7 \mathrm{H}), 7.18-7.14(\mathrm{~m}, 3 \mathrm{H}), 5.16\left(\mathrm{dd}, 1 \mathrm{H},{ }^{2} J_{\mathrm{H}-\mathrm{F}}=\right.$ $\left.48.0 \mathrm{~Hz}, J_{\mathrm{H}-\mathrm{H}}=3.0 \mathrm{~Hz}\right), 4.60\left(\mathrm{dd}, 1 \mathrm{H},{ }^{3} \mathrm{~J}_{\mathrm{H}-\mathrm{F}}=\right.$ $\left.28.0 \mathrm{~Hz}, J_{\mathrm{H}-\mathrm{H}}=3.0 \mathrm{~Hz}\right), 3.61(\mathrm{~s}, 3 \mathrm{H}) .{ }^{19} \mathrm{~F}$ NMR $\left(282 \mathrm{MHz}, \mathrm{CDCl}_{3}\right): \delta-194.2\left(\mathrm{dd},{ }^{2} J_{\mathrm{F}-\mathrm{H}}=48.0\right.$ $\left.\mathrm{Hz},{ }^{3} J_{\mathrm{F}-\mathrm{H}}=28.0 \mathrm{~Hz}\right) \cdot{ }^{13} \mathrm{C}\left\{{ }^{1} \mathrm{H}\right\}$ NMR $(75 \mathrm{MHz}$, $\left.\mathrm{CDCl}_{3}\right): \delta 167.9\left(\mathrm{~d},{ }^{2} \mathrm{~J}_{\mathrm{C}-\mathrm{F}}=30.0 \mathrm{~Hz}\right), 137.8$, 133.6, 132.9, 128.9, 128.7, 128.4, 128.4, 126.2, 127.7, $91.3\left(\mathrm{~d},{ }^{1} \mathrm{~J}_{\mathrm{C}-\mathrm{F}}=187.5 \mathrm{~Hz}\right), 55.8(\mathrm{~d}$, $\left.{ }^{2} J_{C-F}=22.5 \mathrm{~Hz}\right)$, 52.6. IR: 2947, 1755, 1438,
1225, 1087, 1001, 736, 701, 688, 540, $467 \mathrm{~cm}^{-}$ 1. HRMS $\left(\mathrm{API}^{+}\right) \mathrm{m} / \mathrm{z}:[\mathrm{M}+\mathrm{H}]^{+}$calcd for $\mathrm{C}_{16} \mathrm{H}_{16} \mathrm{FO}_{2} \mathrm{~S}: 291.0855$, found: 291.0859 .

(2S,3R)-methyl 2-fluoro-3-phenyl-3(phenylthio)propanoate (anti-20). (E)-methyl 2fluoro-3-phenylacrylate $(0.3 \mathrm{mmol}, 54.4 \mathrm{mg})$, with $(\mathrm{DHQ})_{2} \mathrm{PYR}(0.03 \mathrm{mmol}, 26.4 \mathrm{mg})$ were stirred in neat benzenethiol $(1.5 \mathrm{mmol}, 165.0$ $\mathrm{mg}$ ) at $0{ }^{\circ} \mathrm{C}$ for 8 days according to the general procedure $\mathbf{b} .{ }^{19} \mathrm{~F}$ NMR of crude product showed $d r=24: 76$. The crude was purified by silica gel column chromatography (petroleum ether/EtOAc, from $15 / 1$ to $10 / 1, v / v$ ) affording two diastereoisomers in $56 \%$ yield (major and minor mixture: $49 \mathrm{mg}$ as a white solid). $51 \%$ ee was obtained for the major diastereoisomer [determined by HPLC, IC column, Hept/i-PrOH: $95 / 5$, flow $=1 \mathrm{~mL} / \mathrm{min}, \lambda=230 \mathrm{~nm}$, $\mathrm{t}$ (minor) $=$ $6.773 \mathrm{~min}, \mathrm{t}$ (major) $=7.453 \mathrm{~min}$.]. The racemic product has been obtained following the procedure a (the same scale) in $74 \%$ yield (major: $30 \mathrm{mg}$ as a white solid; major and minor mixture: $34 \mathrm{mg}$ ) with $d r=33: 67$.

${ }^{1} \mathrm{H}$ NMR $\left(300 \mathrm{MHz}, \mathrm{CDCl}_{3}\right): \delta 7.38-7.34(\mathrm{~m}$, $2 \mathrm{H}), 7.25-7.19(\mathrm{~m}, 8 \mathrm{H}), 5.09\left(\mathrm{dd}, 1 \mathrm{H},{ }^{2} J_{\mathrm{H}-\mathrm{F}}=\right.$ $\left.48.0 \mathrm{~Hz}, J_{\mathrm{H}-\mathrm{H}}=3.0 \mathrm{~Hz}\right), 4.56\left(\mathrm{dd}, 1 \mathrm{H},{ }^{3} J_{\mathrm{H}-\mathrm{F}}=\right.$ $\left.28.0 \mathrm{~Hz}, J_{\mathrm{H}-\mathrm{H}}=6.0 \mathrm{~Hz}\right), 3.54(\mathrm{~s}, 3 \mathrm{H}) .{ }^{19} \mathrm{~F}$ NMR $\left(282 \mathrm{MHz}, \mathrm{CDCl}_{3}\right): \delta-195.0\left(\mathrm{dd},{ }^{2} J_{\mathrm{F}-\mathrm{H}}=48.0\right.$ $\left.\mathrm{Hz},{ }^{3} \mathrm{~J}_{\mathrm{F}-\mathrm{H}}=26.0 \mathrm{~Hz}\right) \cdot{ }^{13} \mathrm{C}\left\{{ }^{1} \mathrm{H}\right\} \mathrm{NMR}(75 \mathrm{MHz}$, $\left.\mathrm{CDCl}_{3}\right): \delta 168.0\left(\mathrm{~d},{ }^{2} J_{\mathrm{C}-\mathrm{F}}=22.5 \mathrm{~Hz}\right), 135.5$, $133.5,132.9,129.2,128.9,128.8,128.6$, $128.4,128.4,89.2\left(\mathrm{~d},{ }^{1} \mathrm{~J}_{\mathrm{C}-\mathrm{F}}=195.0 \mathrm{~Hz}\right), 54.6(\mathrm{~d}$, $\left.{ }^{2} J_{\mathrm{C}-\mathrm{F}}=15.0 \mathrm{~Hz}\right), 52.4$.

(2S,3S)-methyl 3-((2-aminophenyl)thio)-2fluoro-3-phenylpropanoate (21). (Z)-methyl 2fluoro-3-phenylacrylate $(0.3 \mathrm{mmol}, 54.4 \mathrm{mg})$, with $(\mathrm{DHQ})_{2} \mathrm{PYR}(0.03 \mathrm{mmol}, 26.4 \mathrm{mg})$ were stirred in neat 2-aminobenzenethiol $(1.5 \mathrm{mmol}$, $188.0 \mathrm{mg}$ ) at room temperature for 4 days according to the general procedure $\mathbf{b}$. ${ }^{19} \mathrm{~F}$ NMR of crude product showed $d r=91: 9$. The crude was purified by silica gel column chromatography (DCM/PE, from $5 / 1$ to $2 / 1$, v/v) affording two diastereoisomers in $22 \%$ yield (major: $16 \mathrm{mg}$ as a yellow solid, major and minor mixture: $4 \mathrm{mg}$ ). $51 \%$ ee was obtained for the major diastereoisomer [determined by HPLC, IC column, Hept/i-PrOH: 90/10, flow $=1$ $\mathrm{mL} / \mathrm{min}, \lambda=254 \mathrm{~nm}, \mathrm{t}$ (minor) $=11.947 \mathrm{~min}, \mathrm{t}$ 
(major) $=14.737$ min.]. The racemic product has been obtained following the procedure a (the same scale) in $46 \%$ yield (major: $34 \mathrm{mg}$ as a yellow solid, major and minor mixture: $8 \mathrm{mg}$ ) with $d r=87: 13$.

$[\alpha]_{\mathrm{D}}^{20}=+111.2\left(\mathrm{c} 1.02, \mathrm{CDCl}_{3}\right) ; \mathrm{Mp} 77-79^{\circ} \mathrm{C}$; ${ }^{1} \mathrm{H}$ NMR $\left(300 \mathrm{MHz}, \mathrm{CDCl}_{3}\right): \delta 7.24-7.21(\mathrm{~m}$, $5 \mathrm{H}), 7.10-7.00(\mathrm{~m}, 2 \mathrm{H}), 6.62-6.47(\mathrm{~m}, 2 \mathrm{H})$, $5.13\left(\mathrm{dd}, 1 \mathrm{H},{ }^{2} J_{\mathrm{H}-\mathrm{F}}=48.0, J_{\mathrm{H}-\mathrm{H}}=3.0 \mathrm{~Hz}\right), 4.48$ (dd, $\left.1 \mathrm{H},{ }^{3} J_{\mathrm{H}-\mathrm{F}}=28.0 \mathrm{~Hz}, J_{\mathrm{H}-\mathrm{H}}=3.0 \mathrm{~Hz}\right), 4.23(\mathrm{~s}$, $2 \mathrm{H}), 3.56(\mathrm{~s}, 3 \mathrm{H}) .{ }^{19} \mathrm{~F} \mathrm{NMR}\left(282 \mathrm{MHz}, \mathrm{CDCl}_{3}\right)$ : $\delta-194.2\left(\mathrm{dd},{ }^{2} J_{\mathrm{F}-\mathrm{H}}=48.0 \mathrm{~Hz},{ }^{3} J_{\mathrm{F}-\mathrm{H}}=28.0 \mathrm{~Hz}\right)$. ${ }^{13} \mathrm{C}\left\{{ }^{1} \mathrm{H}\right\}$ NMR $\left(75 \mathrm{MHz}, \mathrm{CDCl}_{3}\right): \delta 168.1\left(\mathrm{~d},{ }^{2} \mathrm{~J}_{\mathrm{C}}\right.$ $\mathrm{F}=30.0 \mathrm{~Hz}), 149.2,138.1,137.5,130.8,128.6$, 128.3, 128.3, 128.1, 118.3, 115.0, $89.5\left(\mathrm{~d},{ }^{1} J_{\mathrm{C}}\right.$ $\mathrm{F}=195.0 \mathrm{~Hz}), 54.0\left(\mathrm{~d},{ }^{2} \mathrm{~J}_{\mathrm{C}-\mathrm{F}}=22.5 \mathrm{~Hz}\right), 52.5$. IR: 3432, 3327, 2951, 1755, 1609, 1217, 1101, 751, 698, 530, $457 \mathrm{~cm}^{-1}$. HRMS $\left(\mathrm{API}^{+}\right) \mathrm{m} / \mathrm{z}$ : $[\mathrm{M}+\mathrm{H}]^{+}$calcd for $\mathrm{C}_{16} \mathrm{H}_{17} \mathrm{FNO}_{2} \mathrm{~S}$ : 306.0964, found: 306.0964 .

(2S,3S)-methyl 2-fluoro-3-phenyl-3-(mtolylthio)propanoate (22). (Z)-methyl 2-fluoro3-phenylacrylate $(0.3 \mathrm{mmol}, 54.4 \mathrm{mg})$, with $(\mathrm{DHQ})_{2}$ PYR $(0.03 \mathrm{mmol}, 26.4 \mathrm{mg})$ were stirred in neat 3-methybenzenethiol $(1.5 \mathrm{mmol}, 186.3$ $\mathrm{mg}$ ) at room temperature for 5 days according to the general procedure $\mathbf{b} .{ }^{19} \mathrm{~F}$ NMR of crude product showed $d r=87: 13$. The crude was purified by silica gel column chromatography (petroleum ether/EtOAc, from 15/1 to $10 / 1$, v/v) affording two diastereoisomers in $72 \%$ yield (major: $50 \mathrm{mg}$ as a colorless oil, major and minor mixture: $16 \mathrm{mg}$ ). $62 \%$ ee was obtained for the major diastereoisomer [determined by HPLC, IC column, Hept $/ i-\mathrm{PrOH}: 90 / 10$, flow $=1$ $\mathrm{mL} / \mathrm{min}, \lambda=254 \mathrm{~nm}, \mathrm{t}$ (minor) $=6.750 \mathrm{~min}, \mathrm{t}$ (major) $=7.183 \mathrm{~min}$.]. The racemic product has been obtained following the procedure a the same scale) in $89 \%$ yield (major: $45 \mathrm{mg}$ as a colorless oil, major and minor mixture: $37 \mathrm{mg}$ ) with $d r=85: 15$.

$[\alpha]_{D}{ }^{20}=+113.0\left(\right.$ c $\left.0.70, \mathrm{CDCl}_{3}\right) ;{ }^{1} \mathrm{H}$ NMR $(300$ $\left.\mathrm{MHz}, \mathrm{CDCl}_{3}\right): \delta 7.31-7.20(\mathrm{~m}, 5 \mathrm{H}), 7.07-6.94$ $(\mathrm{m}, 4 \mathrm{H}), 5.15\left(\mathrm{dd}, 1 \mathrm{H},{ }^{2} J_{\mathrm{H}-\mathrm{F}}=48.0 \mathrm{~Hz}, J_{\mathrm{H}-\mathrm{H}}=3.0\right.$ $\mathrm{Hz}), 4.59\left(\mathrm{dd}, 1 \mathrm{H},{ }^{3} J_{\mathrm{H}-\mathrm{F}}=28.0 \mathrm{~Hz}, J_{\mathrm{H}-\mathrm{H}}=3.0\right.$ $\mathrm{Hz}), 3.60$ (s, 3H), 2.18 (s, 3H). ${ }^{19} \mathrm{~F}$ NMR (282 $\mathrm{MHz}, \mathrm{CDCl}_{3}$ ): $\delta-194.1$ (dd, ${ }^{2} \mathrm{~J}_{\mathrm{F}-\mathrm{H}}=48.0 \mathrm{~Hz},{ }^{3} \mathrm{~J}_{\mathrm{F}-}$ $\mathrm{H}=28.0 \mathrm{~Hz}) \cdot{ }^{13} \mathrm{C}\left\{{ }^{1} \mathrm{H}\right\}$ NMR $\left(75 \mathrm{MHz}, \mathrm{CDCl}_{3}\right): \delta$ $168.0\left(\mathrm{~d},{ }^{2} \mathrm{~J}_{\mathrm{C}-\mathrm{F}}=22.5 \mathrm{~Hz}\right), 138.7,138.0,133.5$, $133.3,129.8,128.8,128.7,128.6,128.4$, $128.4,128.1,91.3\left(\mathrm{~d},{ }^{1} \mathrm{~J}_{\mathrm{C}-\mathrm{F}}=187.5 \mathrm{~Hz}\right), 55.7(\mathrm{~d}$, $\left.{ }^{2} J_{C-F}=22.5 \mathrm{~Hz}\right), 52.5,21.2$. IR: 2960, 1765, 1741, 1437, 1219, 1101, 774, 692, 544, 436 $\mathrm{cm}^{-1}$. HRMS $\left(\mathrm{API}^{+}\right) \mathrm{m} / \mathrm{z}:[\mathrm{M}+\mathrm{H}]^{+}$calcd for $\mathrm{C}_{17} \mathrm{H}_{18} \mathrm{FO}_{2} \mathrm{~S}: 305.1012$, found: 305.1024.

(2S,3S)-methyl 2-fluoro-3-(phenylthio)-3-(ptolyl)propanoate (23). (Z)-methyl 2-fluoro-3-( $p$ tolyl)acrylate $(0.3 \mathrm{mmol}, 58.3 \mathrm{mg})$, with $(\mathrm{DHQ})_{2} \mathrm{PYR}(0.03 \mathrm{mmol}, 26.4 \mathrm{mg})$ were stirred in neat benzenethiol $(1.5 \mathrm{mmol}, 151.5 \mathrm{mg})$ at 0 ${ }^{\circ} \mathrm{C}$ for 8 days according to the general procedure $\mathbf{b} .{ }^{19} \mathrm{~F}$ NMR of crude product showed $d r=88: 12$. The crude was purified by silica gel column chromatography (petroleum ether/EtOAc, from $15 / 1$ to $10 / 1$, v/v) affording two diastereoisomers in 36\% yield (major: 29 $\mathrm{mg}$ as a white solid, major and minor mixture: $4 \mathrm{mg}$ ). $69 \%$ ee was obtained for the major diastereoisomer [determined by HPLC, IC column, Hept $/ i-\mathrm{PrOH}: 90 / 10$, flow $=1 \mathrm{~mL} / \mathrm{min}$, $\lambda=254 \mathrm{~nm}, \mathrm{t}($ minor $)=6.490 \mathrm{~min}, \mathrm{t}$ (major) $=$ $7.410 \mathrm{~min}$.]. The racemic product has been obtained following the procedure a (the same scale) in $75 \%$ yield (major: $51 \mathrm{mg}$ as a white solid; major and minor mixture: $17 \mathrm{mg}$ ) with $d r$ $=77: 23$.

$[\alpha]_{D}^{20}=+129.6\left(\mathrm{c} 0.57, \mathrm{CDCl}_{3}\right) ; \mathrm{Mp} 82-84^{\circ} \mathrm{C}$; ${ }^{1} \mathrm{H}$ NMR $\left(300 \mathrm{MHz}, \mathrm{CDCl}_{3}\right): \delta 7.27-7.24(\mathrm{~m}$, $2 \mathrm{H}), 7.17-7.13(\mathrm{~m}, 4 \mathrm{H}), 7.05-7.03(\mathrm{~m}, 2 \mathrm{H})$, $5.12\left(\mathrm{dd}, 1 \mathrm{H},{ }^{2} J_{\mathrm{H}-\mathrm{F}}=48.0 \mathrm{~Hz}, J_{\mathrm{H}-\mathrm{H}}=6.0 \mathrm{~Hz}\right)$, $4.57\left(\mathrm{dd}, 1 \mathrm{H},{ }^{3} J_{\mathrm{H}-\mathrm{F}}=28.0 \mathrm{~Hz}, J_{\mathrm{H}-\mathrm{H}}=6.0 \mathrm{~Hz}\right)$, 3.60 (s, 3H), 2.24 (s, 3H). ${ }^{19} \mathrm{~F}$ NMR $(282 \mathrm{MHz}$, $\left.\mathrm{CDCl}_{3}\right): \delta-194.0\left(\mathrm{dd},{ }^{2} J_{\mathrm{F}-\mathrm{H}}=48.0 \mathrm{~Hz},{ }^{3} J_{\mathrm{F}-\mathrm{H}}=\right.$ $28.0 \mathrm{~Hz}) \cdot{ }^{13} \mathrm{C}\left\{{ }^{1} \mathrm{H}\right\} \mathrm{NMR}\left(75 \mathrm{MHz}, \mathrm{CDCl}_{3}\right): \delta$ $168.0\left(\mathrm{~d},{ }^{2} J_{\mathrm{C}-\mathrm{F}}=22.5 \mathrm{~Hz}\right.$ ), 138.0, 134.8, 133.8, 132.8, 129.4, 128.9, 128.3, 128.2, 127.8, 91.5 $\left(\mathrm{d},{ }^{1} \mathrm{~J}_{\mathrm{C}-\mathrm{F}}=195.0 \mathrm{~Hz}\right), 55.5\left(\mathrm{~d},{ }^{2} \mathrm{~J}_{\mathrm{C}-\mathrm{F}}=22.5 \mathrm{~Hz}\right)$, 52.6, 21.2. IR: 2927, 1739, 1434, 1269, 1085, 1008, 741, 570, $413 \mathrm{~cm}^{-1}$. HRMS $\left(\mathrm{API}^{+}\right) \mathrm{m} / \mathrm{z}$ : $[\mathrm{M}+\mathrm{H}]^{+}$calcd for $\mathrm{C}_{17} \mathrm{H}_{18} \mathrm{FO}_{2} \mathrm{~S}: 305.1012$, found: 305.1009 .

(2S,3S)-methyl 3-(2-cyanophenyl)-2-fluoro-3(phenylthio)propanoate (24). (Z)-methyl 3-(2cyanophenyl)-2-fluoroacrylate $(0.3 \mathrm{mmol}, 61.6$ $\mathrm{mg}$ ), with (DHQ) ${ }_{2} \mathrm{PYR}(0.03 \mathrm{mmol}, 26.4 \mathrm{mg})$ were stirred in neat benzenethiol $(1.5 \mathrm{mmol}$, $151.5 \mathrm{mg}$ ) at room temperature for 5 days according to the general procedure $\mathbf{b}$. ${ }^{19} \mathrm{~F}$ NMR of crude product showed $d r=87: 13$. The crude 
was purified by silica gel column chromatography (petroleum ether/EtOAc, from $15 / 1$ to $10 / 1, \mathrm{v} / \mathrm{v})$ affording two diastereoisomers in $78 \%$ yield (major: $56 \mathrm{mg}$ as a white solid, major and minor mixture: $14 \mathrm{mg}$ ). $52 \%$ ee was obtained for the major diastereoisomer [determined by HPLC, IC column, Hept $/ i-\mathrm{PrOH}:$ 90/10, flow $=1 \mathrm{~mL} / \mathrm{min}$, $\lambda=254 \mathrm{~nm}, \mathrm{t}$ (minor) $=12.513 \mathrm{~min}, \mathrm{t}$ (major) $=$ $13.350 \mathrm{~min}$.]. The racemic product has been obtained following the procedure a the same scale) in $62 \%$ yield (major: $43 \mathrm{mg}$ as a white solid; major and minor mixture: $16 \mathrm{mg}$ ) with $d r$ $=89: 11$.

$[\alpha]_{D}^{20}=+3.0\left(\right.$ c 1.03, $\left.\mathrm{CDCl}_{3}\right) ; \mathrm{Mp} 71-72{ }^{\circ} \mathrm{C} ;{ }^{1} \mathrm{H}$ NMR $\left(300 \mathrm{MHz}, \mathrm{CDCl}_{3}\right): \delta 7.74-7.71(\mathrm{~m}, 1 \mathrm{H})$, $7.56-7.51(\mathrm{~m}, 2 \mathrm{H}), 7.35-7.29(\mathrm{~m}, 3 \mathrm{H}), 7.20$ $-7.18(\mathrm{~m}, 3 \mathrm{H}), 5.20\left(\mathrm{dd}, 1 \mathrm{H},{ }^{2} J_{\mathrm{H}-\mathrm{F}}=48.0 \mathrm{~Hz}\right.$, $\left.J_{\mathrm{H}-\mathrm{H}}=3.0 \mathrm{~Hz}\right), 5.12\left(\mathrm{dd}, 1 \mathrm{H},{ }^{3} J_{\mathrm{H}-\mathrm{F}}=28.0 \mathrm{~Hz}, \mathrm{~J}_{\mathrm{H}}\right.$ $\mathrm{H}=3.0 \mathrm{~Hz}), 3.64(\mathrm{~s}, 3 \mathrm{H}) .{ }^{19} \mathrm{~F}$ NMR $(282 \mathrm{MHz}$, $\left.\mathrm{CDCl}_{3}\right): \delta-195.8\left(\mathrm{dd},{ }^{2} J_{\mathrm{F}-\mathrm{H}}=48.0 \mathrm{~Hz},{ }^{3} J_{\mathrm{F}-\mathrm{H}}=\right.$ $28.0 \mathrm{~Hz}) \cdot{ }^{13} \mathrm{C}\left\{{ }^{1} \mathrm{H}\right\}$ NMR $\left(75 \mathrm{MHz}, \mathrm{CDCl}_{3}\right): \delta$ $167.0\left(\mathrm{~d},{ }^{2} \mathrm{~J}_{\mathrm{C}-\mathrm{F}}=22.5 \mathrm{~Hz}\right), 141.4,133.4,133.2$, $132.8,132.1,130.0,129.9,129.2,128.6$, 128.5, 117.0, 112.1, 89.9 (d, $\left.{ }^{1} J_{\mathrm{C}-\mathrm{F}}=195.0 \mathrm{~Hz}\right)$, $53.0\left(\mathrm{~d},{ }^{2} \mathrm{~J}_{\mathrm{C}-\mathrm{F}}=15.0 \mathrm{~Hz}\right.$ ), 52.8. IR: 2947, 2224, 1732, 1438, 1283, 1106, 1016, 743, 692, 555, $491 \mathrm{~cm}^{-1}$. HRMS $\left(\mathrm{API}^{+}\right) \mathrm{m} / \mathrm{z}:[\mathrm{M}+\mathrm{H}]^{+}$calcd for $\mathrm{C}_{17} \mathrm{H}_{15} \mathrm{FNO}_{2} \mathrm{~S}$ : 316.0808 , found: 316.0814 .

(2S,3S)-methyl 3-(3-cyanophenyl)-2-fluoro-3(phenylthio)propanoate (25). (Z)-methyl 3-(3cyanophenyl)-2-fluoroacrylate $(0.3 \mathrm{mmol}, 61.6$ $\mathrm{mg})$, with $(\mathrm{DHQ})_{2} \mathrm{PYR}(0.03 \mathrm{mmol}, 26.4 \mathrm{mg})$ were stirred in neat benzenethiol $(1.5 \mathrm{mmol}$, $151.5 \mathrm{mg}$ ) at room temperature for 5 days according to the general procedure $\mathbf{b} .{ }^{19} \mathrm{~F}$ NMR of crude product showed $d r=92: 8$. The crude was purified by silica gel column chromatography (petroleum ether/EtOAc, from $15 / 1$ to $10 / 1, \mathrm{v} / \mathrm{v})$ affording two diastereoisomers in $76 \%$ yield (major: $60 \mathrm{mg}$ as a colorless oil, major and minor mixture: $8 \mathrm{mg}$ ). $52 \%$ ee was obtained for the major diastereoisomer [determined by HPLC, IC column, Hept $/ i-\mathrm{PrOH}:$ 90/10, flow $=1 \mathrm{~mL} / \mathrm{min}$, $\lambda=254 \mathrm{~nm}, \mathrm{t}$ (minor) $=50.573 \mathrm{~min}, \mathrm{t}$ (major) $=$ $54.183 \mathrm{~min}$.]. The racemic product has been obtained following the procedure a the same scale) in $62 \%$ yield (major: $54 \mathrm{mg}$ as a colorless oil; major and minor mixture: $5 \mathrm{mg}$ ) with $d r=$ 92:8.

$[\alpha]_{D}^{20}=+85.8$ (c 1.15, $\left.\mathrm{CDCl}_{3}\right) ;{ }^{1} \mathrm{H}$ NMR $(300$ $\left.\mathrm{MHz}, \mathrm{CDCl}_{3}\right): \delta 7.56-7.48(\mathrm{~m}, 3 \mathrm{H}), 7.37-7.32$ $(\mathrm{m}, 1 \mathrm{H}), 7.23-7.16(\mathrm{~m}, 5 \mathrm{H}), 5.16\left(\mathrm{dd}, 1 \mathrm{H},{ }^{2} J_{\mathrm{H}-}\right.$ $\left.\mathrm{F}=48.0 \mathrm{~Hz}, J_{\mathrm{H}-\mathrm{H}}=3.0 \mathrm{~Hz}\right), 4.60\left(\mathrm{dd}, 1 \mathrm{H},{ }^{3} \mathrm{~J}_{\mathrm{H}-\mathrm{F}}=\right.$ $\left.28.0 \mathrm{~Hz}, J_{\mathrm{H}-\mathrm{H}}=3.0 \mathrm{~Hz}\right), 3.69(\mathrm{~s}, 3 \mathrm{H}) .{ }^{19} \mathrm{~F} \mathrm{NMR}$ (282 MHz, $\left.\mathrm{CDCl}_{3}\right): \delta-195.4\left(\mathrm{dd},{ }^{2} J_{\mathrm{F}-\mathrm{H}}=48.0\right.$ $\left.\mathrm{Hz},{ }^{3} J_{\mathrm{F}-\mathrm{H}}=28.0 \mathrm{~Hz}\right) \cdot{ }^{13} \mathrm{C}\left\{{ }^{1} \mathrm{H}\right\} \mathrm{NMR}(75 \mathrm{MHz}$, $\left.\mathrm{CDCl}_{3}\right): \delta 167.5\left(\mathrm{~d},{ }^{2} \mathrm{~J}_{\mathrm{C}-\mathrm{F}}=22.5\right.$

$\mathrm{Hz}), 139.7,133.4$, 133.0, 132.9, 132.4, 132.0, $131.8,129.5,129.2,128.5,118.4,112.8,90.7$ $\left(\mathrm{d},{ }^{1} \mathrm{~J}_{\mathrm{C}-\mathrm{F}}=195.0 \mathrm{~Hz}\right), 55.2\left(\mathrm{~d},{ }^{2} \mathrm{~J}_{\mathrm{C}-\mathrm{F}}=15.0 \mathrm{~Hz}\right)$, 52.8. IR: 2953, 2231, 1763, 1438, 1221, 1102, 1008, 801, 739, 690, 528, $479 \mathrm{~cm}^{-1}$. HRMS $\left(\mathrm{API}^{+}\right) \mathrm{m} / \mathrm{z}:[\mathrm{M}+\mathrm{H}]^{+}$calcd for $\mathrm{C}_{17} \mathrm{H}_{15} \mathrm{FNO}_{2} \mathrm{~S}$ : 316.0808, found: 316.0808 .

(2S,3S)-methyl 3-(4-cyanophenyl)-2-fluoro-3(phenylthio)propanoate (26). (Z)-methyl 3-(4cyanophenyl)-2-fluoroacrylate $(0.3 \mathrm{mmol}, 61.6$ $\mathrm{mg}$ ), with (DHQ) ${ }_{2} \mathrm{PYR}(0.03 \mathrm{mmol}, 26.4 \mathrm{mg})$ were stirred in neat benzenethiol $(1.5 \mathrm{mmol}$, $151.5 \mathrm{mg}$ ) at room temperature for 5 days according to the general procedure $\mathbf{b} .{ }^{19} \mathrm{~F}$ NMR of crude product showed $d r=100: 0$. The crude was purified by silica gel column chromatography (petroleum ether/EtOAc, from $15 / 1$ to $10 / 1, v / v)$ affording an only diastereoisomer in $70 \%$ yield $(66 \mathrm{mg}$ as a white solid). $68 \%$ ee was obtained for the major diastereoisomer [determined by HPLC, IC column, Hept $/ i-\mathrm{PrOH}: 90 / 10$, flow $=1 \mathrm{~mL} / \mathrm{min}$, $\lambda=254 \mathrm{~nm}, \mathrm{t}$ (minor) $=21.380 \mathrm{~min}, \mathrm{t}$ (major) $=$ $23.313 \mathrm{~min}$.]. The racemic product has been obtained following the procedure a (the same scale) in $64 \%$ yield (major: $51 \mathrm{mg}$ as a white solid; major and minor mixture: $9 \mathrm{mg}$ ) with $d r=$ 81:19.

$[\alpha]_{D}^{20}=+168.8\left(\mathrm{c} 0.32, \mathrm{CDCl}_{3}\right) ; \mathrm{Mp} 79-80^{\circ} \mathrm{C}$; ${ }^{1} \mathrm{H}$ NMR $\left(300 \mathrm{MHz}, \mathrm{CDCl}_{3}\right): \delta 7.54-7.38(\mathrm{~m}$, $4 \mathrm{H}), 7.23-7.16(\mathrm{~m}, 5 \mathrm{H}), 5.17\left(\mathrm{dd}, 1 \mathrm{H},{ }^{2} \mathrm{~J}_{\mathrm{H}-\mathrm{F}}=\right.$ $\left.48.0 \mathrm{~Hz}, J_{\mathrm{H}-\mathrm{H}}=3.0 \mathrm{~Hz}\right), 4.61\left(\mathrm{dd}, 1 \mathrm{H},{ }^{3} \mathrm{~J}_{\mathrm{H}-\mathrm{F}}=\right.$ $\left.28.0 \mathrm{~Hz}, J_{\mathrm{H}-\mathrm{H}}=3.0 \mathrm{~Hz},\right), 3.70(\mathrm{~s}, 3 \mathrm{H}) .{ }^{19} \mathrm{~F} \mathrm{NMR}$ $\left(282 \mathrm{MHz}, \mathrm{CDCl}_{3}\right): \delta-195.3\left(\mathrm{dd},{ }^{2} J_{\mathrm{F}-\mathrm{H}}=48.0\right.$ $\left.\mathrm{Hz},{ }^{3} \mathrm{~J}_{\mathrm{F}-\mathrm{H}}=28.0 \mathrm{~Hz}\right) .{ }^{13} \mathrm{C}\left\{{ }^{1} \mathrm{H}\right\} \mathrm{NMR}(75 \mathrm{MHz}$, $\left.\mathrm{CDCl}_{3}\right): \delta 167.5\left(\mathrm{~d},{ }^{2} J_{\mathrm{C}-\mathrm{F}}=30.0 \mathrm{~Hz}\right), 143.3$, $133.4,132.4,132.4,129.3,129.3,129.2$, $126.5,118.4,112.1,90.6\left(\mathrm{~d},{ }^{1} J_{\mathrm{C}-\mathrm{F}}=195.0 \mathrm{~Hz}\right)$, $55.6\left(\mathrm{~d},{ }^{2} \mathrm{~J}_{\mathrm{C}-\mathrm{F}}=15.0 \mathrm{~Hz}\right.$ ), 52.9. IR: 2967, 2237, 1767, 1441, 1214, 1096, 1003, 836, 745, 570, 
$437 \mathrm{~cm}^{-1}$. HRMS $\left(\mathrm{API}^{+}\right) \mathrm{m} / \mathrm{z}:[\mathrm{M}+\mathrm{H}]^{+}$calcd for $\mathrm{C}_{17} \mathrm{H}_{15} \mathrm{FNO}_{2} \mathrm{~S}: 316.0808$, found: 316.0790 .

(2S,3S)-methyl 3-(2-aminophenyl)thio)-2fluoro-3-(4-methoxyphenyl)propanoate (27).

(Z)-methyl

2-fluoro-3-(4-

methoxyphenyl)acrylate $(0.3 \mathrm{mmol}, 63.1 \mathrm{mg})$, with $(\mathrm{DHQ})_{2} \mathrm{PYR}(0.03 \mathrm{mmol}, 26.4 \mathrm{mg})$ were stirred in neat 2-aminobenzenethiol $(1.5 \mathrm{mmol}$, $188.0 \mathrm{mg}$ ) at room temperature for 3 days according to the general procedure $\mathbf{b} .{ }^{19} \mathrm{~F}$ NMR of crude product showed $d r=94: 6$. The crude was purified by silica gel column chromatography (DCM/PE, from $5 / 1$ to $2 / 1, \mathrm{v} / \mathrm{v}$ ) affording two diastereoisomers in $37 \%$ yield (major: $30 \mathrm{mg}$ as a yellow solid, major and minor mixture: $7 \mathrm{mg}$ ). $73 \%$ ee was obtained for the major diastereoisomer [determined by HPLC, IC column, Hept/i-PrOH: 90/10, flow = 1 $\mathrm{mL} / \mathrm{min}, \lambda=254 \mathrm{~nm}, \mathrm{t}$ (minor) $=16.977 \mathrm{~min}, \mathrm{t}$ (major) $=23.033 \mathrm{~min}$.]. The racemic product has been obtained following the procedure a (the same scale) in $40 \%$ yield (major: $35 \mathrm{mg}$ as a yellow solid, major and minor mixture: $5 \mathrm{mg}$ ) with $d r=87: 13$.

$[\alpha]_{D}^{20}=+183.2\left(\mathrm{c} 2.20, \mathrm{CDCl}_{3}\right) ; \mathrm{Mp} 80-82{ }^{\circ} \mathrm{C}$; ${ }^{1} \mathrm{H}$ NMR $\left(300 \mathrm{MHz}, \mathrm{CDCl}_{3}\right): \delta 7.18-6.99(\mathrm{~m}$, $4 \mathrm{H}), 6.76-6.73(\mathrm{~m}, 2 \mathrm{H}), 6.62-6.59(\mathrm{~m}, 1 \mathrm{H})$, $6.52-6.47(\mathrm{~m}, 1 \mathrm{H}), 5.09\left(\mathrm{dd}, 1 \mathrm{H},{ }^{2} J_{\mathrm{H}-\mathrm{F}}=48.0\right.$ $\left.\mathrm{Hz}, J_{\mathrm{H}-\mathrm{H}}=3.0 \mathrm{~Hz}\right), 4.44\left(\mathrm{dd}, 1 \mathrm{H},{ }^{3} J_{\mathrm{H}-\mathrm{F}}=28.0 \mathrm{~Hz}\right.$, $\left.J_{\mathrm{H}-\mathrm{H}}=3.0 \mathrm{~Hz}\right), 4.25(\mathrm{~s}, 2 \mathrm{H}), 3.71(\mathrm{~s}, 3 \mathrm{H}), 3.54$ (s, 3H). ${ }^{19} \mathrm{~F}$ NMR (282 MHz, $\left.\mathrm{CDCl}_{3}\right): \delta-193.9$ (dd, ${ }^{2} J_{F-H}=48.0 \mathrm{~Hz},{ }^{3} J_{F-H}=28.0 \mathrm{~Hz}$ ). ${ }^{13} \mathrm{C}\left\{{ }^{1} \mathrm{H}\right\}$ NMR $\left(75 \mathrm{MHz}, \mathrm{CDCl}_{3}\right): \delta 168.1\left(\mathrm{~d},{ }^{2} \mathrm{~J}_{\mathrm{C}-\mathrm{F}}=22.5\right.$ $\mathrm{Hz}), 159.3,149.2,137.5,130.8,130.1,129.5$, $129.5,118.3,115.2,115.0,113.9,91.0\left(\mathrm{~d},{ }^{1} \mathrm{~J}_{\mathrm{c}}\right.$ $\mathrm{F}=195.0 \mathrm{~Hz}), 55.3,53.4\left(\mathrm{~d},{ }^{2} \mathrm{~J}_{\mathrm{C}-\mathrm{F}}=22.5 \mathrm{~Hz}\right)$, 52.5. IR: $3354,2953,1754,1605,1511,1480$, 1243, 1102, 1023, 827, 743, 534, $435 \mathrm{~cm}^{-1}$. HRMS $\left(\mathrm{ESI}^{+}\right) \quad \mathrm{m} / \mathrm{z}: \quad[\mathrm{M}+\mathrm{H}]^{+}$calcd for $\mathrm{C}_{17} \mathrm{H}_{19} \mathrm{FNO}_{3} \mathrm{~S}: 336.1070$, found: 336.1062 .

\section{(2S,3S)-3-fluoro-2-phenyl-2,3-}

dihydrobenzo[b][1,4]thiazepin-4(5H)-one (28).

Compound 21 (0.35 mmol, $106.8 \mathrm{mg}$,) with $p$ $\mathrm{TsOH}(0.07 \mathrm{mmol}, 6.9 \mathrm{mg})$ were added into 2 $\mathrm{mL}$ xylene and the resulting mixture was heated to reflux overnight. After completion of the reaction, the solvent was removed in vacuo and the crude mixture was purified by column chromatography (petroleum ether/EtOAc, from5/1 to $4 / 1, v / v$ ) to give the desired product (28) in $85 \%$ yield (81 mg, white solid). $51 \%$ ee [determined by HPLC, AD-H column, Hept $/ i$ $\mathrm{PrOH}: 80 / 20$, flow $=1 \mathrm{~mL} / \mathrm{min}, \lambda=254 \mathrm{~nm}, \mathrm{t}$ $($ minor $)=10.467 \mathrm{~min}, \mathrm{t}$ (major) $=14.543 \mathrm{~min}$. $[\alpha]_{D}^{23}=+126.1$ (c 0.80, $\left.\mathrm{CDCl}_{3}\right) ; \mathrm{Mp} 161-163$ ${ }^{\circ} \mathrm{C} ;{ }^{1} \mathrm{H}$ NMR $\left(300 \mathrm{MHz}, \mathrm{CDCl}_{3}\right): \delta 9.39(\mathrm{~s}, 1 \mathrm{H})$, $7.74\left(\mathrm{~d}, 1 \mathrm{H}, J_{\mathrm{H}-\mathrm{H}}=6.0 \mathrm{~Hz}\right), 7.59-7.58(\mathrm{~m}, 2 \mathrm{H})$, $7.45-7.35(\mathrm{~m}, 4 \mathrm{H}), 7.27-7.21(\mathrm{~m}, 2 \mathrm{H}), 5.36$ $-5.18(\mathrm{~m}, 2 \mathrm{H}) .{ }^{19} \mathrm{~F}$ NMR $\left(282 \mathrm{MHz}, \mathrm{CDCl}_{3}\right): \delta-$ $190.5\left(\mathrm{dd},{ }^{2} \mathrm{~J}_{\mathrm{F}-\mathrm{H}}=47.9 \mathrm{~Hz},{ }^{3} \mathrm{~J}_{\mathrm{F}-\mathrm{H}}=8.5 \mathrm{~Hz}\right.$ ). ${ }^{13} \mathrm{C}\left\{{ }^{1} \mathrm{H}\right\}$ NMR $\left(75 \mathrm{MHz}, \mathrm{CDCl}_{3}\right): \delta 169.6\left(\mathrm{~d},{ }^{2} \mathrm{~J}_{\mathrm{C}}\right.$ $\mathrm{F}=22.5 \mathrm{~Hz}), 140.3,135.3,135.3,134.8,130.6$, 129.3, 128.8, 128.5, 126.7, 126.5, 123.1, 88.8 $\left(\mathrm{d},{ }^{1} J_{\mathrm{C}-\mathrm{F}}=195.0 \mathrm{~Hz}\right), 56.3\left(\mathrm{~d},{ }^{2} J_{\mathrm{C}-\mathrm{F}}=15.0 \mathrm{~Hz}\right)$. IR: 3188, 3072, 2961, 2903, 1687, 1474, 1306, 1075, 768, 736, 717, 696, 524, $463 \mathrm{~cm}^{-1}$. HRMS $\left(\mathrm{API}^{+}\right) \quad \mathrm{m} / \mathrm{z}: \quad[\mathrm{M}+\mathrm{H}]^{+}$calcd for $\mathrm{C}_{15} \mathrm{H}_{13}$ FNOS: 274.0702, found: 274.0707.

(2S,3S)-3-fluoro-2-(4-methoxyphenyl)-2,3dihydrobenzo[b][1,4]thiazepin-4(5H)-one (29). Compound $27(0.2 \mathrm{mmol}, 67.0 \mathrm{mg}$,) with $p$ $\mathrm{TsOH}(0.04 \mathrm{mmol}, 3.9 \mathrm{mg})$ were added into 1.2 $\mathrm{mL}$ xylene and the resulting mixture was heated to reflux overnight. After completion of the reaction, the solvent was removed in vacuo and the crude mixture was purified by column chromatography (petroleum ether/EtOAc, from5/1 to $3 / 1, v / v$ ) to give the desired product 29 in $76 \%$ yield (46 mg, white solid). 73\% ee [determined by HPLC, AD-H column, Hept $/ i$ $\mathrm{PrOH}: 80 / 20$, flow $=1 \mathrm{~mL} / \mathrm{min}, \lambda=254 \mathrm{~nm}, \mathrm{t}$ $($ minor $)=13.940 \mathrm{~min}, \mathrm{t}$ (major $)=21.507 \mathrm{~min}$. $[\alpha]_{D}{ }^{23}=+122.2\left(\right.$ c 0.54, $\left.\mathrm{CDCl}_{3}\right) ; \mathrm{Mp} 165-166$ ${ }^{\circ} \mathrm{C} ;{ }^{1} \mathrm{H}$ NMR $\left(300 \mathrm{MHz}, \mathrm{CDCl}_{3}\right): \delta 9.00(\mathrm{~s}, 1 \mathrm{H})$, $7.63\left(\mathrm{~d}, 1 \mathrm{H}, J_{\mathrm{H}-\mathrm{H}}=9.0 \mathrm{~Hz}\right), 7.41-7.30(\mathrm{~m}, 3 \mathrm{H})$, $7.18-7.11(\mathrm{~m}, 2 \mathrm{H}), 6.81-6.75(\mathrm{~m}, 2 \mathrm{H}), 5.22$ - $5.04(\mathrm{~m}, 2 \mathrm{H}), 3.71$ (s, 3H). ${ }^{19} \mathrm{~F}$ NMR (282 $\mathrm{MHz}, \mathrm{CDCl}_{3}$ ): $\delta-190.4$ (ddd, ${ }^{2} J_{\mathrm{F}-\mathrm{H}}=47.9 \mathrm{~Hz}$, $\left.{ }^{3} \mathrm{~J}_{\mathrm{F}-\mathrm{H}}=8.5 \mathrm{~Hz},{ }^{4} J_{\mathrm{F}-\mathrm{H}}=2.8 \mathrm{~Hz}\right) \cdot{ }^{13} \mathrm{C}\left\{{ }^{1} \mathrm{H}\right\} \operatorname{NMR}(75$ $\left.\mathrm{MHz}, \mathrm{CDCl}_{3}\right): \delta 169.3\left(\mathrm{~d},{ }^{2} \mathrm{~J}_{\mathrm{C}-\mathrm{F}}=22.5 \mathrm{~Hz}\right.$ ), $160.0,140.3,134.8,130.5,130.5,126.8$, $123.1,113.9,88.4\left(\mathrm{~d},{ }^{1} \mathrm{~J}_{\mathrm{C}-\mathrm{F}}=195.0 \mathrm{~Hz}\right), 55.6(\mathrm{~d}$, $\left.{ }^{2} J_{\mathrm{C}-\mathrm{F}}=22.5 \mathrm{~Hz}\right)$, 55.3. IR: 3189, 3076, 2923, 2856, 1684, 1513, 1475, 1306, 1250, 1083, 1027, 785, 764, 652, 490, $410 \mathrm{~cm}^{-1}$. HRMS $\left(\mathrm{API}^{+}\right) \mathrm{m} / \mathrm{z}:[\mathrm{M}+\mathrm{H}]^{+}$calcd for $\mathrm{C}_{16} \mathrm{H}_{15} \mathrm{FNO}_{2} \mathrm{~S}$ : 304.0808, found: 304.0812 . 
(2S,3S)-5-(2-(dimethylamino)ethyl)-3-fluoro-2phenyl-2,3-dihydrobenzo[b][1,4]thiazepin-

4(5H)-one (30).

To a solution of compound $28(0.25 \mathrm{mmol}, 68.5$ $\mathrm{mg}$ ) in ethyl acetate $(1.5 \mathrm{~mL})$ was added 2dimethylaminoethyl chloride hydrochloride (72.0 $\mathrm{mg}, 0.50 \mathrm{mmol}$ ), followed by potassium carbonate $(138.0 \mathrm{mg}, 1.0 \mathrm{mmol})$ and $\mathrm{H}_{2} \mathrm{O}(15$ $\mu \mathrm{L})$. After the mixture was stirred for $24 \mathrm{~h}$ under reflux, it was cooled to room temperature. The mixture was dried over $\mathrm{Na}_{2} \mathrm{SO}_{4}$ then the crude mixture was purified by column chromatography (DCM/MeOH, $10 / 1$ to $8 / 1, \mathrm{v} / \mathrm{v}$ ) to give the desired product 30 in $41 \%$ yield (35 $\mathrm{mg}$, white solid). $51 \%$ ee, [determined by HPLC, luxcell 2 column, Hept/i-PrOH: 80/20, flow $=1 \mathrm{~mL} / \mathrm{min}, \lambda=254 \mathrm{~nm}, \mathrm{t}$ (minor) $=16.503$ $\min , \mathrm{t}$ (major) $=19.920 \mathrm{~min}$.]

$[\alpha]_{D}^{20}=+144.9$ (c 0.31, $\left.\mathrm{CDCl}_{3}\right) ; \mathrm{Mp} 165-166$ ${ }^{\circ} \mathrm{C} ;{ }^{1} \mathrm{H}$ NMR $\left(300 \mathrm{MHz}, \mathrm{CDCl}_{3}\right): \delta 7.84-7.73$ $(\mathrm{m}, 1 \mathrm{H}), 7.59-7.28(\mathrm{~m}, 8 \mathrm{H}), 5.43-4.90(\mathrm{~m}$, $2 \mathrm{H}), 4.53-4.26(\mathrm{~m}, 1 \mathrm{H}), 3.85-3.71(\mathrm{~m}, 1 \mathrm{H})$, $2.79-2.45(\mathrm{~m}, 2 \mathrm{H}), 2.29(\mathrm{~s}, 6 \mathrm{H}) .{ }^{19} \mathrm{~F}$ NMR $(282$ $\left.\mathrm{MHz}, \mathrm{CDCl}_{3}\right): \delta-189.3\left(\mathrm{~d},{ }^{2} \mathrm{~J}_{\mathrm{F}-\mathrm{H}}=47.9 \mathrm{~Hz}\right)$. ${ }^{13} \mathrm{C}\left\{{ }^{1} \mathrm{H}\right\}$ NMR $\left(75 \mathrm{MHz}, \mathrm{CDCl}_{3}\right): \delta 166.5\left(\mathrm{~d},{ }^{2} \mathrm{~J}_{\mathrm{C}}\right.$ $\mathrm{F}=22.5 \mathrm{~Hz}), 145.1,135.5,131.1,129.9,128.9$, 128.5, 128.4, 128.2, 128.1, 127.6, 124.7, 86.2 $\left(\mathrm{d},{ }^{1} \mathrm{~J}_{\mathrm{C}-\mathrm{F}}=187.5 \mathrm{~Hz}\right), 56.7,55.0\left(\mathrm{~d},{ }^{2} \mathrm{~J}_{\mathrm{C}-\mathrm{F}}=22.5\right.$ $\mathrm{Hz})$, 47.8, 45.6. IR: 3064, 2939, 2765, 1681, 1441, 1179, 1088, 856, 766, 602, 591, $428 \mathrm{~cm}^{-}$ ${ }^{1}$.HRMS $\left(\mathrm{ESI}^{+}\right) \mathrm{m} / \mathrm{z}:[\mathrm{M}+\mathrm{H}]^{+}$calcd for $\mathrm{C}_{19} \mathrm{H}_{22} \mathrm{FN}_{2} \mathrm{OS}: 345.1437$, found: 345.1449 .

(2S,3S)-5-(2-(dimethylamino)ethyl)-3-fluoro-2(4-methoxyphenyl)-2,3-

dihydrobenzo[b][1,4]thiazepin-4(5H)-one (31). To a solution of compound $29(0.15 \mathrm{mmol}, 45.0$ $\mathrm{mg})$ in ethyl acetate $(1.0 \mathrm{~mL})$ was added 2dimethylaminoethyl chloride hydrochloride (43.2 $\mathrm{mg}, 0.30 \mathrm{mmol}$ ), followed by potassium carbonate $(82.8 \mathrm{mg}, 0.6 \mathrm{mmol})$ and $\mathrm{H}_{2} \mathrm{O}(10$ $\mu \mathrm{L}$ ). After the mixture was stirred for $24 \mathrm{~h}$ under reflux, it was cooled to room temperature. The mixture was dried over $\mathrm{Na}_{2} \mathrm{SO}_{4}$ then the crude mixture was purified by column chromatography (DCM/MeOH, 9/1 to $8 / 1$, v/v) to give the desired product 31 in $32 \%$ yield (18 $\mathrm{mg}$, colorless oil). $73 \%$ ee, [determined by HPLC, luxcell 2 column, Hept $/ i-\mathrm{PrOH}: 80 / 20$, flow $=1.2 \mathrm{~mL} / \mathrm{min}, \lambda=254 \mathrm{~nm}, \mathrm{t}$ (minor) $=$ $18.370 \mathrm{~min}, \mathrm{t}$ (major) $=23.353 \mathrm{~min}$.]
$[\alpha]_{\mathrm{D}}{ }^{20}=+126.4\left(\mathrm{c} 0.47, \mathrm{CDCl}_{3}\right) ;{ }^{1} \mathrm{H}$ NMR $(300$ $\left.\mathrm{MHz}, \mathrm{CDCl}_{3}\right): \delta 7.72-7.64(\mathrm{~m}, 1 \mathrm{H}), 7.43-7.35$ $(\mathrm{m}, 3 \mathrm{H}), 7.23-7.19(\mathrm{~m}, 2 \mathrm{H}), 6.86-6.75(\mathrm{~m}$, $2 \mathrm{H}), 5.28-4.78(\mathrm{~m}, 2 \mathrm{H}), 4.45-4.17(\mathrm{~m}, 1 \mathrm{H})$, $3.74(\mathrm{~s}, 3 \mathrm{H}), 3.71-3.62(\mathrm{~m}, 1 \mathrm{H}), 2.71-2.32$ $(\mathrm{m}, 2 \mathrm{H}), 2.21(\mathrm{~s}, 6 \mathrm{H}) .{ }^{19} \mathrm{~F}$ NMR $(282 \mathrm{MHz}$, $\left.\mathrm{CDCl}_{3}\right): \delta-189.2\left(\mathrm{~d},{ }^{2} J_{\mathrm{F}-\mathrm{H}}=47.9 \mathrm{~Hz}\right) \cdot{ }^{13} \mathrm{C}\left\{{ }^{1} \mathrm{H}\right\}$ NMR $\left(75 \mathrm{MHz}, \mathrm{CDCl}_{3}\right): \delta 166.6\left(\mathrm{~d},{ }^{2} \mathrm{~J}_{\mathrm{C}-\mathrm{F}}=22.5\right.$ $\mathrm{Hz}), 160.0,145.0,135.4,131.0,129.4,128.2$, 127.6, 124.6, 113.9, 86.1 (d, ${ }^{1} J_{\mathrm{C}-\mathrm{F}}=187.5 \mathrm{~Hz}$ ), $56.6,55.3,54.5\left(\mathrm{~d},{ }^{2} J_{\mathrm{C}-\mathrm{F}}=22.5 \mathrm{~Hz}\right), 47.7,45.5$. IR: 3058, 2938, 2770, 1676, 1609, 1511, 1251, 1178, 1092, 1029, 732, 602, $407 \mathrm{~cm}^{-1}$. HRMS $\left(\mathrm{ESI}^{+}\right) \mathrm{m} / \mathrm{z}:[\mathrm{M}+\mathrm{H}]^{+}$calcd for $\mathrm{C}_{20} \mathrm{H}_{24} \mathrm{FN}_{2} \mathrm{O}_{2} \mathrm{~S}$ : 375.1543, found: 375.1532 .

\section{Acknowledgements}

This work was partially supported by Normandie Université (NU), the Région Normandie, the Centre National de la Recherche Scientifique (CNRS), Université de Rouen Normandie (URN), INSA Rouen Normandie, Labex SynOrg (ANR-11-LABX-0029), and Innovation Chimie Carnot (I2C). X. H. thanks the CSC (China Scholarship Council) for a doctoral fellowship.

\section{References}

[1] (a) Kirsch, P. in Modern Fluoroorganic Chemistry: Synthesis, Reactivity, Applications, Wiley-VCH: Weinheim, 2013. (b) Bégué, J.-P.; Bonnet-Delpon, D. in Bioorganic and Medicinal Chemistry of Fluorine, John Wiley \& Sons, Inc.: Hoboken, NJ, 2008. (c) Handbook of Fluoropolymer Science and Technology, (Eds: D. W. Smith, S. T. lacono, S. S. Iyer), John Wiley \& Sons, Inc.: Hoboken, NJ, 2014. (d) Wang, J.; Sanchez-Rosello, M.; Acena, J. L.; del Pozo, C.; Sorochinsky, A. E.; Fustero, S.; Soloshonok, V. A.; Liu, H. Fluorine in Pharmaceutical Industry: Fluorine-Containing Drugs Introduced to the Market in the Last Decade (2001-2011). Chem. Rev. 2014, 114, 2432-2506. (e) Fluorine in Life Sciences: Pharmaceuticals, Medicinal Diagnostics, and Agrochemicals, 1st ed., Progress in Fluorine Science Series, (Eds.: G. Haufe, F. Leroux), Elsevier, Academic Press, 2018. (f) Meanwell, N. A. Fluorine and Fluorinated Motifs in the Design and Application of Bioisosteres for Drug Design. J. Med. Chem. 2018, 61, 5822-5880. 
[2] (a) Qiu, X.-L.; Yue, X.; Qing, F.-L. in Chiral Drugs: Chemistry and Biological Action (Eds.: G.-Q. Lin, Q.-D. You, J.-F. Cheng), John Wiley \& Sons, Inc.: Hoboken, NJ, 2011, pp. 195-251. (b) Cahard, D.; Xu, X.; Couve-Bonnaire, S.; Pannecoucke, $X$. Fluorine \& chirality: how to create a nonracemic stereogenic carbonfluorine centre? Chem. Soc. Rev. 2010, 39, 558-568. (c) Shibata, N.; Ishimaru, T.; Nakamura, S.; Toru, T. New approaches to enantioselective fluorination: Cinchona alkaloids combinations and chiral ligands/metal complexes. J. Fluorine Chem. 2007, 128, 469483. (d) Ma, J.; Cahard, D. Asymmetric fluorination, trifluoromethylation, and perfluoroalkylation Reactions. Chem. Rev. 2004, 104, 6119-6146.

[3] (a) Asymmetric Organocatalysis 1, Science of Synthesis series (Ed.: B. List), Thieme, Stuttgart, 2012 (b) Asymmetric Organocatalysis 2, Science of Synthesis series (Ed.: K. Maruoka), Thieme, Stuttgart, 2012. (c) Alemán, J.; Cabrera, S. Applications of asymmetric organocatalysis in medicinal chemistry. Chem. Soc. Rev. 2013, 42, 774793.

[4] (a) Heravi, M. M.; Hajiabbasi, P. Recent advances in C-heteroatom bond forming by asymmetric Michael addition. Mol. Diversity 2014, 18, 411-439. (b) Vicario, J. L.; Badia, D.; Carrillo, L. Organocatalytic enantioselective Michael and hetero-Michael reactions. Synthesis 2007, 2065-2092. (c) Enders, D.; Luttgen, K.; Narine, A. A. Asymmetric sulfaMichael additions. Synthesis 2007, 959-980. (d) Enders, D.; Wang, C.; Liebich, J. X. Organocatalytic asymmetric aza-Michael additions. Chem. Eur. J. 2009, 15, 1105811076. (e) Nising, C. F.; Brase, S. Recent developments in the field of oxa-Michael reactions. Chem. Soc. Rev. 2012, 41, 988-999. (f) Tsogoeva, S. B. Recent Advances in Asymmetric organocatalytic 1,4-conjugate additions. Eur. J. Org. Chem. 2007, 1701-1716.

[5] (a) Lemonnier, G.; Zoute, L.; Dupas, G.; Quirion, J.-C.; Jubault, P. Diethylzinc-mediated one-step stereoselective synthesis of $\alpha$ fluoroacrylates from aldehydes and ketones. two different pathways depending on the carbonyl partner. J. Org. Chem. 2009, 74, 4124-4131. (b) Rousée, K.; Bouillon, J.-P.; Couve-Bonnaire, S.; Pannecoucke, X. Stereospecific synthesis of tri- and tetrasubstituted a-fluoroacrylates by MizorokiHeck reaction. Org. Lett. 2016, 18, 540-543.

[6] (a) Hudlicky, M. The synthesis of $\gamma$ fluoroglutamic acid. Tetrahedron Lett. 1960, 14, 21-22. (b) Tsushima, T.; Kawada, K.; Ishihara, S.; Uchida, N.; Shiratori, O.; Higaki, J.; Hirata, M. Fluorine containing amino acids and their derivatives. 7. Synthesis and antitumor activity of $\alpha$ - and $\gamma$-substituted methotrexate analogs. Tetrahedron 1988, 44, 5375-5387. (c) Belokon, Y. N.: Maleev, V. I.: Savel'eva, T. F.; Moskalenko, M. A.; Pripadchev, D. A.; Khrustalev, V. N.; Saghiyan A. S. Asymmetric synthesis of enantiomerically and diastereoisomerically enriched 4-[F or $\mathrm{Br}]-$ substituted glutamic acids. Amino Acids 2010, 39, 1171-1176.

[7] (a) Elkik, E.; Dahan, R.; Parlier, A. Fluoroalkylation and annelation of cyclic $\beta$ diketones. Bull. Soc. Chim. Fr. 1981, 9, 13531360. (b) Ramb, D. C.; Lerchen, A.; Kischkewitz, M.; Beutel, B.; Fustero, S.; Haufe, G. Addition of nucleophiles to fluorinated Michael acceptors. Eur. J. Org. Chem. 2016, 1751-1759.

[8] Calata, C.; Pfund, E.; Lequeux, T. Toward the Synthesis of benzothiazolyl fluoroaminosulfones. J. Org. Chem. 2009, 74, 9399-9405.

[9] Chuit, C.; Sauvêtre, R.; Masure, D.; Normant, J. F. Réaction du diméthylcuprate de lithium avec les dérivés carbonylés $\alpha, \beta$ insaturés $\alpha$-fluorés et $\alpha$-chlorés. Tetrahedron 1979, 35, 2645-2653.

[10] Yoshikai, N.; Nakamura, E. Mechanisms of Nucleophilic organocopper(I) reactions. Chem. Rev. 2012, 112, 2339-2372.

[11] Shi, G.-Q.; Wang, Q.; Schlosser, M. Versatile 2-fluoroacrylic building blocks for the synthesis of fluorinated heterocyclic compounds. Tetrahedron 1996, 52, 44034410. 
[12] (a) Chauhan, P.; Mahajan, S.; Enders, D. Organocatalytic carbon-sulfur bond-forming reactions. Chem. Rev. 2014, 114, 8807-8864. (b) Nair, D. P.; Podgórski, M.; Chatani, S.; Gong, T.; Xi, W.; Fenoli, C. R.; Bowman, C. N. The thiol-Michael addition click reaction: $A$ powerful and widely used tool in materials Chemistry. Chem. Mater. 2014, 26, 724-744. (c) Hoyle, C. E.; Lowe, A. B.; Bowman, C. N. Thiol-click chemistry: a multifaceted toolbox for small molecule and polymer synthesis. Chem. Soc. Rev. 2010, 39, 1355-1387.

[13] (a) Feng, M.; Tang, B.; Liang, S.; Jiang, X. Sulfur containing scaffolds in drugs: synthesis and application in medicinal chemistry. Curr. Top. Med. Chem. 2016, 16, 1200-1216. (b) Dunbar, K. L.; Scharf, D. H.; Litomska, A.; Hertweck, C. Enzymatic carbon-sulfur bond formation in natural product biosynthesis. Chem. Rev. 2017, 117, 5521-5577. (c) Jana, M.; Misra, A. K. Stereoselective synthesis of $\beta$ glycosyl thiols and their synthetic applications. J. Org. Chem. 2013, 78, 2680-2686; d) Fontecave, M.; Ollagnier-de-Choudens, S.; Mulliez, E. Biological radical sulfur insertion reactions. Chem. Rev. 2003, 103, 2149-2166.

[14] (a) Pracejus, H.; Wilke, F.-W.; Hanemann, K. Asymmetrisch katalysierte additionen von thiolen an $\alpha$-aminoacrylsäure- derivate und nitroolefine. J. Prakt. Chem. 1977, 319, 219229. (b) Kumar, A.; Salunkhe, R. V.; Rane, R. A.; Dike, S. Y. Novel catalytic enantioselective protonation (proton transfer) in Michael addition of benzenethiol to $\alpha$-acrylacrylates: synthesis of $(S)$-naproxen and a-arylpropionic acids or esters. J. Chem. Soc., Chem. Commun. 1991, 485-486. (c) Leow, D.; Lin, S.; Chittimalla, S. K.; Fu, X.; Tan, C.-H. Enantioselective protonation catalyzed by a chiral bicyclic guanidine derivative. Angew. Chem. Int. Ed. 2008, 47, 5641-5645. (d) Farley, A. J. M.; Sandford, C.; Dixon, D. J. Bifunctional iminophosphorane catalyzed enantioselective sulfa-Michael addition to unactivated $\alpha$ substituted acrylate esters. J. Am. Chem. Soc. 2015, 137, 15992-15995.

[15] (a) Yamashita, H.; Mukaiyama, T. Asymmetric Michael addition of thiophenol to maleic acid esters. Chem. Lett. 1985, 14, 363-
366. (b) Dong, X.-Q.; Fang, X.; Wang, C.-J. Organocatalytic Asymmetric sulfa-Michael addition of thiols to 4,4,4-trifluorocrotonates. Org. Lett. 2011, 13, 4426-4429. (c) Fang, X.; Li, J.; Wang, C.-J. Organocatalytic asymmetric sulfa-Michael addition of thiols to $\alpha, \beta$ unsaturated hexafluoroisopropyl esters: expeditious access to $(R)$-thiazesim. Org. Lett. 2013, 15, 3448-3451. (d) Yang, J.; Farley, A. J. M.; Dixon, D. J. Enantioselective bifunctional iminophosphorane catalyzed sulfa-Michael addition of alkyl thiols to unactivated $\beta$ substituted- $\alpha, \beta$-unsaturated esters. Chem. Sci. 2017, 8, 606-610.

[16] (a) Keniya, J.; Natu, A. A.; Gogte, V. N. An enantioselective synthesis of $(R)$ and $(S)$ phenylalanine. Chem. Ind. (London) 1986, 243. (b) Dodda, R.; Mandal, T.; Zhao, C.-G. Organocatalytic highly enantioselective tandem Michael-Knoevenagel reaction for the synthesis of substituted thiochromanes. Tetrahedron Lett. 2008, 49, 1899-1902. (c) Choudhury, A. R.: Mukherjee, S. A catalytic Michael/Horner-Wadsworth-Emmons

cascade reaction for enantioselective synthesis of thiochromenes. Adv. Synth. Catal. 2013, 355, 1989-1995.

[17] See the Supporting Information.

[18] (a) Cinchona alkaloids in synthesis \& catalysis (Ed.: C. E. Song), Wiley-VCH: Weinheim, 2009. (b) Tian, S.-K.; Chen, Y.; Hang, J.; Tang, L.; Mcdaid, P.; Deng, L. Asymmetric organic catalysis with modified Cinchona alkaloids. Acc. Chem. Res. 2004, 37, 621-631. (c) Marcelli, T.; Hiemstra, H. Cinchona alkaloids in asymmetric organocatalysis. Synthesis 2010, 1229-1279. (d) Singh, G. S.; Yeboah, E. M. O. Recent applications of Cinchona alkaloid-based catalysts in asymmetric addition reactions. Reports in Organic Chemistry 2016, 6, 47-75.

[19] (a) Boratyński, P. J. Dimeric Cinchona alkaloids. Mol Divers 2015, 19, 385-422. (b) Mcdaid, P.; Chen, Y.; Deng, L. A highly enantioselective and general conjugate addition of thiols to cyclic enones with an organic Catalyst. Angew. Chem. Int. Ed. 2002, 41, 338-340. (c) Kowalczyk, R.; Wierzba, A. J.; 
Boratyński, P. J.; Bąkowicz, J. Enantioselective conjugate addition of aliphatic thiols to divergently activated electron poor alkenes and dienes. Tetrahedron 2014, 70, 5834-5842.

[20] (a) Okino, T.; Hoashi, Y.; Takemoto Y. Enantioselective Michael reaction of malonates to nitroolefins catalyzed by bifunctional organocatalysts. J. Am. Chem. Soc. 2003, 125, 12672-12673. (b) Li, B.-J.; Jiang L.; Liu, M.; Chen, Y.-C.; Ding, L.-S.; Wu, Y. Asymmetric Michael addition of arylthiols to $\alpha, \beta$ unsaturated carbonyl compounds catalyzed by bifunctional organocatalysts. Synlett 2005, 603-606. (c) Vakulya, B.; Varga, S.; Csámpai, A.; Soós, T. Highly enantioselective conjugate addition of nitromethane to chalcones using bifunctional Cinchona organocatalysts. Org. Lett. 2005, 7, 1967-1969. (d) Li, H.; Zu, L.; Wang, J.; Wang, W. Organocatalytic enantioselective Michael addition of thioacetic acid to enones. Tetrahedron Lett. 2006, 47, 3145-3148. (e) Rana, N. K.; Selvakumar, S.; Singh, V. K. Highly enantioselective organocatalytic sulfa-Michael addition to $\alpha, \beta$ unsaturated ketones. J. Org. Chem. 2010, 75, 2089-2091.

[21] (a) CCDC 2014985 (13) contains the supplementary crystallographic data for this paper. (b) CCDC 2014986 (18) contains the supplementary crystallographic data for this paper. These data can be obtained free of charge from the Cambridge Crystallographic Data Centre.

[22] Miyata, O.; Shinada, T.; Naito, T.; Ninimiya, I. Stereospecific nucleophilic addition reaction of thiophenol to $\alpha, \beta$-unsaturated esters. Chem. Pharm. Bull. 1989, 39, 31583160.

[23] Schwartz, A.; Madan, P. B.; Mohacsi, E.; O'Brien, J. P.; Todaro, L. J.; Coffen, D. L. Enantioselective synthesis of calcium channel blockers of the diltiazem group. J. Org. Chem. 1992, 57, 851-856.

[24] Bariwal, J. B.; Upadhyay, K. D.; Manvar, A. T.; Trivedi, J. C.; Singh, J. S.; Jain, K. S.; Shah, A. K. 1,5-Benzothiazepine, a versatile pharmacophore: A review. Eur. J. Med. Chem. 2008, 43, 2279-2290.

Table of contents

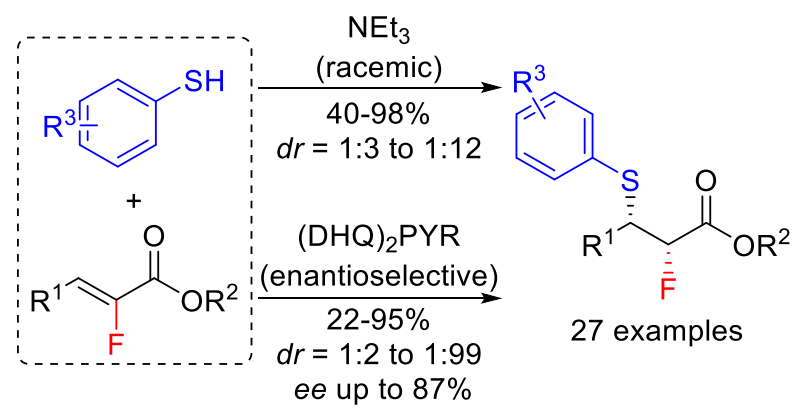

